\title{
CSI 2264: CHARACTERIZING ACCRETION-BURST DOMINATED LIGHT CURVES FOR YOUNG STARS IN NGC 2264*
}

\author{
John Stauffer ${ }^{1}$, Ann Marie Cody ${ }^{1}$, Annie Baglin $^{2}$, Silvia Alencar $^{3}$, Luisa Rebull ${ }^{1}$, Lynne A. Hillenbrand $^{4}$, \\ Laura Venuti ${ }^{5}$, Neal J. Turner ${ }^{6}$, John Carpenter ${ }^{4}$, Peter Plavchan ${ }^{7}$, Krzysztof Findeisen $^{4}$, Sean Carey $^{1}$, \\ Susan Terebey $^{8}$, María Morales-Calderón ${ }^{9}$, Jerome Bouvier $^{5}$, Giusi Micela ${ }^{10}$, Ettore Flaccomio ${ }^{10}$, Inseok Song ${ }^{11}$, \\ Rob Gutermuth $^{12}$, Lee Hartmann ${ }^{13}$, Nuria Calvet ${ }^{13}$, Barbara Whitney ${ }^{14}$, David Barrado ${ }^{9}$, Frederick J. Vrba ${ }^{15}$, \\ Kevin Covey $^{16}$, William Herbst ${ }^{17}$, Gabor Furesz $^{18}$, Suzanne Aigrain $^{19}$, and Fabio Favata ${ }^{20}$ \\ ${ }^{1}$ Spitzer Science Center, California Institute of Technology, Pasadena, CA 91125, USA; stauffer@ipac.caltech.edu \\ ${ }^{2}$ LESIA, Observatoire de Paris-Meudon, 5 place Jules Janssen, F-92195, Meudon, France \\ ${ }^{3}$ Departamento de Física-ICEx-UFMG, Av. Antônio Carlos, 6627, 30270-901, Belo Horizonte, MG, Brazil \\ ${ }^{4}$ Astronomy Department, California Institute of Technology, Pasadena, CA 91125, USA \\ ${ }^{5}$ UJF-Grenoble 1/CNRS-INSU, Institut de Planétologie et d'Astrophysique de Grenoble (IPAG) UMR 5274, Grenoble, F-38041, France \\ ${ }^{6}$ Jet Propulsion Laboratory, California Institute of Technology, Pasadena, CA 91109, USA \\ ${ }^{7}$ Infrared Processing and Analysis Center, California Institute of Technology, Pasadena, CA 91125, USA \\ ${ }^{8}$ Department of Physics and Astronomy, 5151 State University Drive, California State University at Los Angeles, Los Angeles, CA 90032, USA \\ ${ }^{9}$ Centro de Astrobiología, Dpto. de Astrofísica, INTA-CSIC, P.O. Box 78, E-28691, ESAC Campus, Villanueva de la Cañada, Madrid, Spain \\ ${ }_{10}^{10}$ INAF - Osservatorio Astronomico di Palermo, Piazza del Parlamento 1, I-90134, Palermo, Italy \\ ${ }^{11}$ Department of Physics and Astronomy, The University of Georgia, Athens, GA 30602-2451, USA \\ ${ }^{12}$ Five College Astronomy Department, Smith College, Northampton, MA 01063, USA \\ ${ }^{13}$ Department of Astronomy, University of Michigan, 500 Church Street, Ann Arbor, MI 48105, USA \\ ${ }^{14}$ Astronomy Department, University of Wisconsin-Madison, 475 North Charter Street, Madison, WI 53706, USA \\ ${ }^{15}$ U.S. Naval Observatory, Flagstaff Station, 10391 West Naval Observatory Road, Flagstaff, AZ 86001, USA \\ ${ }^{16}$ Lowell Observatory, 1400 West Mars Hill Road, Flagstaff, AZ 86001, USA \\ ${ }^{17}$ Astronomy Department, Wesleyan University, Middletown, CT 06459, USA \\ ${ }^{18}$ Harvard-Smithsonian Center for Astrophysics, Cambridge, MA 02138, USA \\ ${ }^{19}$ Sub-department of Astrophysics, Department of Physics, University of Oxford, Oxford OX1 3RH, UK \\ ${ }^{20}$ European Space Agency, 8-10 rue Mario Nikis, F-75738 Paris Cedex 15, France \\ Received 2013 November 9; accepted 2014 January 21; published 2014 March 13
}

\begin{abstract}
Based on more than four weeks of continuous high-cadence photometric monitoring of several hundred members of the young cluster NGC 2264 with two space telescopes, NASA's Spitzer and the CNES CoRoT (Convection, Rotation, and planetary Transits), we provide high-quality, multi-wavelength light curves for young stellar objects whose optical variability is dominated by short-duration flux bursts, which we infer are due to enhanced mass accretion rates. These light curves show many brief-several hours to one day-brightenings at optical and near-infrared wavelengths with amplitudes generally in the range of 5\%-50\% of the quiescent value. Typically, a dozen or more of these bursts occur in a 30 day period. We demonstrate that stars exhibiting this type of variability have large ultraviolet (UV) excesses and dominate the portion of the $u-g$ versus $g-r$ color-color diagram with the largest UV excesses. These stars also have large $\mathrm{H} \alpha$ equivalent widths, and either centrally peaked, lumpy $\mathrm{H} \alpha$ emission profiles or profiles with blueshifted absorption dips associated with disk or stellar winds. Light curves of this type have been predicted for stars whose accretion is dominated by Rayleigh-Taylor instabilities at the boundary between their magnetosphere and inner circumstellar disk, or where magneto-rotational instabilities modulate the accretion rate from the inner disk. Among the stars with the largest UV excesses or largest H $\alpha$ equivalent widths, light curves with this type of variability greatly outnumber light curves with relatively smooth sinusoidal variations associated with long-lived hot spots. We provide quantitative statistics for the average duration and strength of the accretion bursts and for the fraction of the accretion luminosity associated with these bursts.
\end{abstract}

Key words: accretion, accretion disks - circumstellar matter - stars: formation - stars: pre-main sequence - stars: variables: T Tauri, Herbig Ae/Be

Online-only material: color figures

\footnotetext{
* Based on data from the Spitzer and CoRoT missions, as well as the Canada-France-Hawaii Telescope (CFHT) MegaCam CCD, and the European Southern Observatory Very Large Telescope, Paranal Chile, under program 088.C-0239. The CoRoT space mission was developed and is operated by the French space agency CNES, with participation of ESA's RSSD and Science Programmes, Austria, Belgium, Brazil, Germany, and Spain. MegaCam is a joint project of CFHT and CEA/DAPNIA, which is operated by the National Research Council (NRC) of Canada, the Institute National des Sciences de l'Univers of the Centre National de la Recherche Scientifique of France, and the University of Hawaii.
}

\section{INTRODUCTION}

Time series photometry obtained with space-based telescopes-MOST (Microvariability and Oscillations of Stars; Walker et al. 2003), CoRoT (Convection, Rotation, and planetary Transits; Baglin et al. 2009), and Kepler-has recently been used to derive very high quality light curves for normal low-mass field stars. Those data have provided a wealth of new information on, for example, the size evolution and stability of cold spots on main-sequence stars (Silva-Valio \& Lanza 2011; Walkowicz et al. 2013), the ages of main-sequence stars from 
asteroseismology (Silva Aguirre et al. 2013), and the frequency of occurrence of planets (Fressin et al. 2013). These results required the very high quality data achievable from space because the variability amplitudes for these old stars are quite small, and in many cases, the needed cadence and duration to successfully address these topics cannot be readily obtained with ground-based data.

Very young, pre-main-sequence (PMS) stars are in principle much more favorable targets for time series photometry. In the first place, the amplitudes of variability for young stellar objects (YSOs) are often much larger, with amplitudes up to a magnitude or more. In addition, there are many more physical processes that can cause variability in YSOs (variable extinction, variable accretion, flares, very large cold spots, relatively rapid rotation, etc.). The different mechanisms have differing timescales, ranging from hours to weeks. They also have different spectral signatures, giving impetus to multi-wavelength and spectral monitoring. The rich variety of phenomena in YSOs have motivated ground-based observing campaigns to characterize their variability properties at least for the past half century.

For the past two decades, the primary reference for the characterization and interpretation of YSO light curves has been Herbst et al. (1994, hereafter H94). H94 sorted YSO light curves into four basic types. Class I light curves are periodic and roughly sinusoidal in shape, and they result from the rotational modulation of cold, non-axisymmetrically distributed starspots; stars with Class I light curves are typically weak-lined T Tauri stars (WTTSs), though some classical T Tauri stars (CTTSs) may also show light curves of this type. Class II light curves are thought to be the result of variations in the veiling continuum of CTTSs, and to reflect changes in the size, shape, or effective temperature of hot spots on their surfaces due to variations in the accretion rate from their circumstellar disks. Most Class IIs vary irregularly in time, with no obvious periodicity, but with characteristic timescales less than a few days. However, a subset of the Class IIs-designated as Class IIp-are periodic, presumably due to a dominant hot spot group whose lifetime is long compared to the star's rotation period. Class III light curves show (generally) irregularly spaced flux dips on an otherwise relatively slowly varying quiescent brightness maximum; these stars generally show little or no veiling in their spectra. Stars with Class III light curves in the H94 sample were all relatively high mass PMS stars with spectral types earlier than K2. H94 was least certain about the physical mechanism for the Class III light curves, but advocated variable extinction by circumstellar material as the most likely cause.

Perhaps the most interesting of these light-curve types is the Class II (and IIp) group, because their properties should provide direct insight into the accretion process. Recent advances in computational astrophysics have made it possible to begin to construct physically realistic 3D magnetohydrodynamic (MHD) models of accretion disks (Kulkarni \& Romanova 2008, hereafter KR08; Romanova et al. 2012, hereafter R12; McKinney et al. 2012; Cemeljic et al. 2013). For stars with relatively strong magnetic dipoles, these models predict that the accreting gas flows along field lines and impacts the star at nearly free-fall velocities at relatively high latitude, resulting in the stable, long-lived hot spots and predicting light curves that are periodic and approximately sinusoidal (see Figure 7(a) of KR08). If one of these "funnel flows" passes through our line of sight, the infalling warm gas could be the source for variability in the inverse P Cygni profiles often seen in the emission line profiles of classical T Tauri stars (Kurosawa \& Romanova 2013). Stars with this type of accretion could plausibly be associated with the Class IIp light curves of H94 (as originally suggested by Bertout et al. 1988).

Other theoretical models predict less well-behaved accretion variability, plausibly better matched to the Class II light curves of H94. Some 3D MHD model simulations from KR08 exhibit Rayleigh-Taylor (RT) instabilities at the disk-magnetosphere boundary, resulting in many short-lived tongues of matter penetrating between the stellar magnetic field lines. Typically, a few such tongues reach the surface of the star at any one time, producing short-lived hot spots at lower latitudes than for the funnel flows and relatively chaotic looking light curves (see Figure 7(b) of KR08). Other 3D MHD models show that in some cases magneto-rotational instabilities (MRIs) in the inner circumstellar disk of a YSO can lead to highly variable accretion rates and predicted light-curve shapes similar to those arising from the RT instabilities (see Figure 6 of R12).

Quantitative comparison between these theoretical model light curves and those of actual YSOs, particularly for the Class IIs, is more difficult than it might appear. That is because the amplitudes predicted for the instability-driven accretion bursts are often only $5 \%-10 \%$, and their typical durations are of order a day. Typical ground-based campaigns with one or two epochs per night can detect this type of variability, but does not provide the cadence, regularity, or photometric accuracy to accurately measure such features. Higher cadence observations are needed in order to clearly characterize the physical mechanisms. Do the light curves of the majority of real CTTSs, in fact, look anything like the model predictions? If so, what types of quantitative information can be drawn from these light curves-for example, what are the relative frequencies of periodic, sinusoidal light curves as would result from stable funnel flows at high latitudes, versus aperiodic, chaotic light curves resulting from RT or MRI driven accretion? Do the aperiodic, chaotic light curves dominate at high accretion rates, as predicted by KR08? Are the amplitudes and durations of the observed accretion bursts roughly in accord with the theoretical predictions?

The first steps toward clarifying how YSOs actually accrete using high-cadence, space-based monitoring have in fact been published recently. Alencar et al. (2010) provided CoRoT monitoring for the YSOs of NGC 2264, and suggested that a few of their CTTS light curves were likely due to stochastic accretion (see their Figure 1(e)). Rucinski et al. (2008) used MOST to obtain a well-sampled light curve for TW Hya, which also showed numerous brief flux excesses that they associated with a variable accretion rate. A small sample of other bright YSOs was also observed with MOST (Siwak et al. 2011; Cody et al. 2013); for the YSOs with disks in this sample, there was some short timescale variability that could not be attributed to long-lived spots or flares, and which could be evidence for variable accretion. However, none of these studies were accompanied by simultaneous spectroscopic or multiwavelength time-series data, which limited the ability to provide conclusive interpretations to the light curves.

We have recently conducted an observational campaign for the star-forming region NGC 2264 designed to have the cadence, duration, precision, wavelength coverage, and sensitivity to resolve many of the long-standing issues for characterizing YSO variability. That program is called the Coordinated Synoptic Investigation of NGC 2264, or CSI 2264. This campaign was built around 30+ days of continuous, simultaneous monitoring by three space telescopes_CoRoT (Baglin et al. 2009), MOST 
(Walker et al. 2003), and Spitzer (Werner et al. 2004). Here, we report one result from that campaign: the empirical identification of a set of YSOs whose light curves are best explained as being dominated by short-timescale variations in the star's accretion rate. We demonstrate that these stars include the most actively accreting members of NGC 2264.

\section{DATA USED FOR THIS PAPER}

Cody et al. (2014) provide a detailed description of all of the optical and infrared (IR) observational data we have obtained for the young stars in NGC 2264 and our basic data reduction procedures. That paper also provides an overview of the lightcurve morphologies we find for the CTTSs in the cluster. Readers are referred to that paper for specific details concerning the observations. Here we provide just a brief synopsis of the observations in order to provide the context necessary for our discussion.

Spitzer's Infrared Array Camera (IRAC; Fazio et al. 2004) was used as part of the CSI 2264 program (program ID 80040; PI: J. Stauffer) to observe NGC 2264 nearly continuously from 2011 December 3 to 2012 January 1. In the first week of that period, approximately four days were spent in "staring mode," where the telescope was pointed to a position near NGC 2264IRS-1 (i.e., near the center of the Spokes cluster; Teixeira et al. 2006) and repeated frames were taken at that position for blocks of 19-26 hr without interruption. The integration time for each exposure was $4.4 \mathrm{~s}$. The two IRAC fields of view are separated on the sky by about $7^{\prime}$, so we collect these light curves for one set of 283 stars in Channel $1(3.6 \mu \mathrm{m})$, and for a disjoint set of 249 stars in Channel $2(4.5 \mu \mathrm{m})$. The relative photometric accuracy for these light curves approaches $1 \mathrm{mmag}$ for stars that are bright but not saturated. For the remaining observing time within the CSI 2264 program, Spitzer observations of NGC 2264 were done in mapping mode, where a rectangular region centered near $(\alpha, \delta) \sim 064045.0,+0940$ (2000) and about $45^{\prime}$ by $40^{\prime}$ in size was observed about 12 times per day, with a total integration time of about $40 \mathrm{~s}$ per point on the sky. For one or two of these maps each day, the data were taken in high-dynamic range mode, resulting also in data with $1.6 \mathrm{~s}$ total integration time. These data provide typical $1 \sigma$ uncertainties of about $0.01 \mathrm{mag}$ for sufficiently bright stars. Most stars have light curves in both channels, but stars near the edge of the map may have data in only one channel.

The CoRoT camera has a field of view in excess of one square degree, which is larger than the physical extent of NGC 2264. However, only stars included within an input catalog have their photometry downlinked to the ground, and these stars must satisfy brightness, crowding, and nebular background constraints. In total, about 490 probable cluster members and more than a 1000 likely field stars were included in the input catalog for the 2011 December observing campaign. Data were obtained from MJD 55,896.8 through MJD 55,935.5, with only a brief interruption around MJD 55,917 due to a telescope pointing problem. For most stars, the photometry is provided at a cadence of $512 \mathrm{~s}$; for a small subset of the stars, data are provided at $32 \mathrm{~s}$ cadence. While there is no hard limit, stars fainter than $R=16.5$ were generally excluded from the target list in order to allow reasonably good signal-to-noise ratio for the CoRoT light curves.

In principle, CoRoT can provide multi-color light curves for some of the observed stars. However, for our purposes, we have only utilized the "white-light" data. CoRoT's bandpass is essentially a broad $V+R$ filter. The CoRoT data are sky- subtracted aperture photometries that have been run through a pipeline designed to mitigate artifacts that are present in the downlinked data (Samadi et al. 2006). In some cases, jumps in the photometry are still present even after this pipeline has been run. When possible, we have removed these jumps manually; in other cases where the jumps are less obvious, we have left the data "as is." The flux rms over $512 \mathrm{~s}$ in the light curves at $R=$ 12 is typically about 0.002 , increasing to about 0.015 at $R=16$.

A 20 day CoRoT "short run" for NGC 2264 was also obtained in 2008 March (Alencar et al. 2010). Light curves for many of the CTTS members were also obtained in that campaign, and we use those data where relevant.

We obtained high-resolution spectra of most of the NGC 2264 members using either Hectochelle on the Multiple Mirror Telescope (MMT; Szentgyorgyi et al. 2011) or the FLAMES multi-object spectrograph on the Very Large Telescope (VLT; Pasquini et al. 2002). The Hectochelle spectra have been discussed previously in Furesz et al. (2006); the observations were obtained in 2004 and 2005, have a resolution of about 34,000 , and cover the spectral range 6460-6650 . In general, no sky background has been subtracted from these profiles, which can leave narrow emission cores from the $\mathrm{H}$ II region at the cluster rest velocity in the stellar spectra. The FLAMES spectra were obtained with UT2 at the VLT, using the HR15N grating, producing a resolution of about 17,000, and covering the spectral range $6375-6815 \AA$. Only six of the stars that are the focus of this paper have FLAMES spectra, but for those stars we have up to 22 spectra, with usually five or six of them obtained during the time period when CoRoT was obtaining photometry of the cluster (and the others being obtained within about 60 days following the CoRoT observations). Spectroscopic rotational velocities $(v \sin i)$ for many of the NGC 2264 members are available from either from Baxter et al. (2009) ${ }^{21}$ or from our analysis of the VLT/FLAMES spectra.

We also use a variety of broad- and narrowband photometry of NGC 2264 members in order to measure the ultraviolet (UV) excesses of the YSOs and thereby identify those that are actively accreting matter from their circumstellar disks, and to measure their spectral energy distributions (SEDs) and thereby characterize the IR excesses from their disks. The UV excess determinations are made using ugri photometry obtained in 2010 and 2012 with the Canada-France-Hawaii Telescope (CFHT) Megacam (L. Venuti et al. 2014, in preparation). The broadband photometry used for the SED fits comes from a variety of sources, but is primarily reported in either Rebull et al. (2002) or Sung et al. (2009).

\section{IDENTIFICATION AND INITIAL CHARACTERIZATION OF YSOS WITH BURST-DOMINATED OPTICAL LIGHT CURVES}

\subsection{Identification of Burst-Dominated Light Curves}

Cold spots on the photosphere, clumps or warps in the inner disk that occult our line of sight, and companions that transit our line of sight to the primaries all produce light curves with periodic signals. These types of variables have been found in abundance by previous ground-based surveys of star-forming regions (e.g., Rebull et al. 2002; Lamm et al. 2004, hereafter L04; Herbst et al. 1994, 2000; Stassun et al. 1999; Grankin et al. 2007;

\footnotetext{
21 Values for $v \sin i$ for just 97 stars were published in Baxter et al. (2009); however, those authors derived $v \sin i$ for a larger set of stars from the same MMT Furesz et al. spectra, and those additional $v \sin i$ values were made available to us by K. Covey.
} 
etc.). As long as the duration of the monitoring period is long enough, and the period is sufficiently stable, one can make up for the relative sparseness of the sampling and the interruptions by weather in these ground surveys by folding the light curve at the identified period. We also find these types of variables in our data, and we provide an initial summary of their prevalence and characteristics in Cody et al. (2014) and L. Affer et al. (2014, in preparation). Rotation periods derived from the 2008 CoRoT NGC 2264 observations are reported in Affer et al. (2013).

Types of variability that are not periodic (e.g., the Class II variables of H94) are much harder to identify and characterize using ground-based photometric monitoring data. The most extensive ground-based photometric monitoring campaign conducted for the YSOs of NGC 2264 was that of L04. Those authors obtained 88 epochs of $I$-band photometry for about 600 cluster members, plus additional candidate members and field stars, during an approximately 40 day campaign. Roughly 400 of these stars, primarily weak-lined T Tauri stars (WTTSs), were identified as periodic variables, and 180 stars, primarily CTTSs, as irregular variables. L04 surmised that in most cases the irregular variability could be attributed to "variable mass accretion resulting in hot spots which are not stable in brightness, size and location over a few rotation periods." However, the small number of epochs and irregular cadence did not allow further elucidation of the physical nature of the variability.

We have examined our data, and find that only 39 of the L04 irregular variables have CoRoT light curves; the overlap is not better primarily because the L04 survey went considerably deeper than the CoRoT data. Twenty of the thirty-nine stars in common with L04 stars have light curves that are heterogeneous in their properties and where more than one physical mechanism may contribute significantly to the light-curve shape. However, for the other half, the light curves fall into two dominant lightcurve types. Ten of the stars have light curves characterized as having a more or less stable light maximum interspersed with well-defined flux dips. Based on our previous experience with these types of light curves (Bouvier et al. 2007; Alencar et al. 2010), we believe that the flux dips in these stars are due to periods of enhanced extinction, as for the Class III variables of H94; in some cases, the flux dips occur approximately periodically and may be due to an inner disk warp passing through our line of sight (Terquem \& Papaloizou 2000). Nine other stars, however, have light curves that appear roughly similar to each other but are dominated by short-duration flux bursts, which we believe are the stars with variable mass accretion that L04 had hoped to identify. These nine light curves appear reasonably similar to those predicted by KR08 or Romanova et al. (2012) for instability-driven accretion. Figure 1 shows CoRoT light curves for three stars from each of these two classes.

In order to determine how common light curves of the type shown in Figures 1(d)-(f) are, two of us (J.R.S. and A.M.C.) have closely examined the CoRoT light curves of all $\sim 550$ probable NGC 2264 members and the $\sim 1250$ Spitzer light curves for cluster members. The CoRoT data provide light curves for 197 CTTSs likely to have ongoing accretion, with nearly $1000 \mathrm{hr}$ of monitoring per star. Within these light curves, we find dozens of YSOs with some evidence of short-duration flux bursts like those shown in Figures 1(d)-(f), though in some cases the flux bursts are superposed with other types of variability, often of larger amplitude. To restrict ourselves to stars whose light curves are dominated by flux bursts, we require the light curves to have: (1) a relatively flat or only slowly varying "continuum"; (2) an intrinsic noise level in the light curve less than 1\%; and (3) presence in the light curve of at least half a dozen narrow (one hour to one day), approximately symmetric (unlike flares; see Section 3.2 and Section 6.1), sharply peaked flux "bursts," with at least one of the bursts having an amplitude greater than $5 \%$ of the continuum level. We have identified a set of 23 YSOs whose 2008 and/or 2011 CoRoT light-curve variability satisfies these criteria. Hereafter, we refer to light curves with these characteristics as burst-dominated light curves. $^{22}$ Table 1 provides the list of the YSOs displaying such light curves and some of their spectroscopic properties. Table 2 provides single-epoch optical, near-IR, and Spitzer photometry for these stars. The CoRoT light curves for all of the stars in Table 1 are provided in the Appendix (Figure 21). For the stars of Table 1 where we also have IRAC light curves, Figure 22 of the Appendix overplots the CoRoT and Spitzer light curves.

In the next several sub-sections, we discuss qualitative aspects of these light curves. The remaining sections will be used to provide more quantitative analysis of these stars and their light curves.

\subsection{Proof That These Flux Bursts Are Real}

Given the comparative novelty of the light curves in Figures 1(d)-(f), it seems useful to begin by providing arguments why we are certain that the flux bursts are real and not due to some type of artifact in the CoRoT data. As described in Cody et al. (2014), not only do we have CoRoT light curves for 550 NGC 2264 members (from both 2008 and 2011), but we also have light curves for more than 500 field stars of similar magnitude obtained simultaneously with the NGC 2264 members and reduced in the same manner. Events such as those shown in Figures 1(d)-(f) and in the Appendix (Figure 21) are not present among the field stars. They are also not present, with one or two possible exceptions, among the NGC 2264 members that do not have accretion disks nor strong emission lines (i.e., among the WTTSs).

For a few of the stars in Table 1, we have four days of highcadence IRAC data simultaneous with a portion of the CoRoT light curves. Figure 2 overplots the IRAC and CoRoT light curves for two of these stars. The close correspondence between the two sets of data provides strong confirmation of the reality of the flux bursts.

As we show in Section 4, based on their ugri photometry, the stars of Table 1 all have UV excesses indicative of active accretion. More significantly, for the set of NGC 2264 YSOs for which we have CoRoT light curves, more than half of the stars with the largest UV excesses are included in Table 1.

\subsection{Resolving Individual Flux Bursts}

The light curves shown in Figures 1(d)-(f) are good at illustrating the existence of the flux bursts. However, because

\footnotetext{
22 While this paper and Cody et al. (2014) are being submitted together and are closely linked, they were written in parallel and evolved somewhat independently. The two papers used slightly different sets of data-most importantly, Stauffer et al. included stars with only 2008 CoRoT light curves, whereas such stars were excluded from Cody et al.-and slightly different criteria for defining light-curve classes. This resulted in somewhat different sets of stars belonging to each class. In particular, the burst-dominated class in Table 1 of this paper includes 23 stars; 19 of those are also listed as burst-dominated in Table 3 of Cody et al. Of the remaining four, one (Mon-000185) was included here based on its 2008 CoRoT light curve and hence was not in the parent sample for the Cody et al. paper. The other three were all classified as having "stochastic" light curves in Cody et al. Inclusion or exclusion of these stars from the burst-dominated class would not appreciably change the conclusions in either paper.
} 

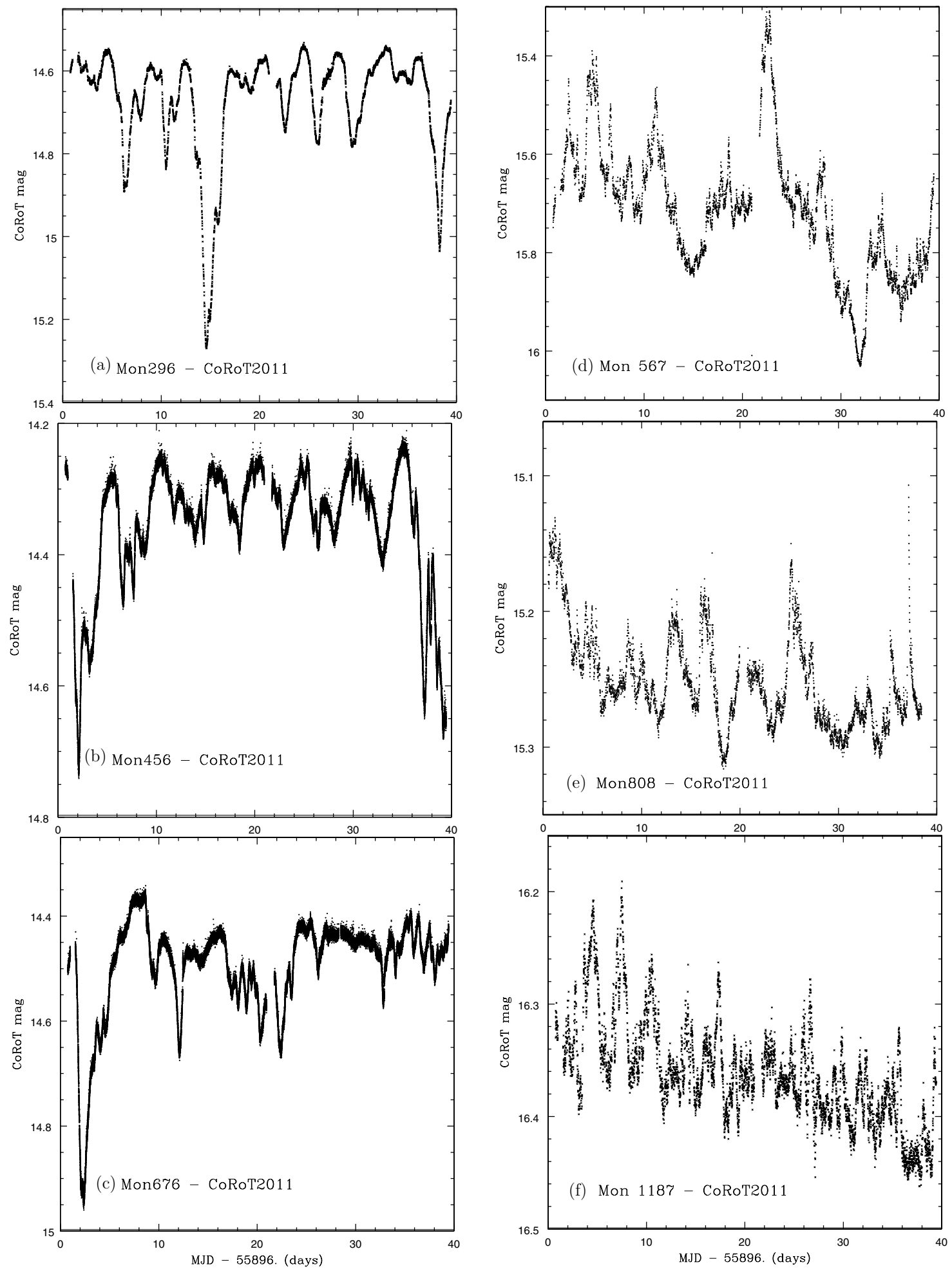

Figure 1. CoRoT light curves for six CTTS members identified by L04 as irregular variables. We identify the first three (Mon-000296, panel (a); Mon-000456, panel (b); and Mon-000676, panel (c)) as having light curves dominated by variable extinction events. The next three stars (Mon-000567, panel (d); Mon-000808, panel (e); and Mon-001187, panel (f)) have light curves dominated by short-duration flux bursts. Each light curve covers roughly 40 days of time; the $y$-axis is in CoRoT counts, where the CoRoT bandpass corresponds roughly to a very broad $V+R$ band filter. For two of the stars (Mon-000456 and Mon-000676) the cadence is roughly every $30 \mathrm{~s}$; for the four other stars, the cadence is about 7.5 minutes.

of the scale of those plots, they do not illustrate well the full structure of those bursts. Figure 3 shows expanded views of portions of two of those light curves. In each case, what appear to be single bursts break up into many blended, shorter duration bursts. The shortest duration bursts visible in Figure 3 last a few hours. Given the standard CoRoT cadence of about 7.5 minutes and typical signal-to-noise ratio, such data could not resolve bursts of significantly shorter duration. See Section 6 for a more quantitative discussion of the burst timescales.

We note for completeness the following. Many stellar flares are detected among the NGC 2264 CoRoT light curves, particularly among the WTTSs. These are usually distinguishable 
Table 1

Basic Information for YSOs with Burst-dominated CoRoT Light Curves

\begin{tabular}{|c|c|c|c|c|c|c|c|c|c|}
\hline Mon ID ${ }^{\mathrm{a}}$ & 2MASS ID & CoRoT 2008 & CoRoT 2011 & $\mathrm{SpT}^{\mathrm{c}}$ & $\begin{array}{c}\mathrm{H} \alpha \mathrm{EW}^{\mathrm{c}} \\
(\AA)\end{array}$ & Н $\alpha$ Type & $\begin{array}{c}v \sin i^{\mathrm{d}} \\
\left(\mathrm{km} \mathrm{s}^{-1}\right)\end{array}$ & Veiling $^{\mathrm{e}}$ & $\operatorname{FR}(4.5)^{\mathrm{f}}$ \\
\hline Mon-000007 & $06415304+0958028$ & 223994721 & 223994721 & K7 & 11.5 & $\mathrm{I}$ & 80.8 & $\cdots$ & 3.62 \\
\hline Mon-000011 & $06411725+0954323$ & 223985009 & 223985009 & K7 & 58.3 & $\cdots$ & $\cdots$ & $\cdots$ & 5.14 \\
\hline Mon-000117 & $06405413+0948434$ & $\ldots$ & 602095753 & M2.5 & 353. & I & $\cdots$ & $\cdots$ & 2.42 \\
\hline Mon-000185 & $06413876+0932117$ & 500007249 & 616919566 & $\mathrm{~K} 4$ & 58.6 & I & 9.6 & $\ldots$ & 3.68 \\
\hline Mon-000260* & $06411099+0935556$ & 500007727 & $\ldots$ & K7 & 61.5 & III-B & 17.5 & 1.99 & 6.60 \\
\hline Mon-000341* & $06405426+0949203$ & 500007473 & 616849439 & M0.5 & 161. & I & 16.2 & 0.74 & 4.22 \\
\hline Mon-000406 & $06405968+0928438$ & $\ldots$ & 616943998 & $\cdots$ & 46.1 & $\cdots$ & $\cdots$ & $\cdots$ & 3.78 \\
\hline Mon-000412 & $06404711+0932401$ & $\cdots$ & 616919737 & M1 & 30.7 & $\mathrm{I}$ & 8.9 & $\cdots$ & 2.56 \\
\hline Mon-000469 & $06404114+0933578$ & $\cdots$ & 602083890 & K7 & 236.5 & III-B & $\cdots$ & $\cdots$ & 4.40 \\
\hline Mon-000474 & $06410682+0927322$ & $\cdots$ & 603396438 & G & 104.7 & I & 17.7 & $\cdots$ & 4.97 \\
\hline Mon-000510* & $06410429+0924521$ & 500007335 & 602079845 & M0 & 101.8 & I & 18. & 0.30 & 3.67 \\
\hline Mon-000567 & $06405639+0935533$ & $\ldots$ & 616919752 & K3 & 84.1 & III-B & $\ldots$ & $\ldots$ & 5.75 \\
\hline Mon-000808 & $06405159+0928445$ & $\ldots$ & 603396401 & $\mathrm{~K} 4$ & 50.2 & I & 9.0 & $\cdots$ & 2.38 \\
\hline Mon-000860 & $06415492+0942527$ & 223995308 & $\ldots$ & M2.5 & 261.0 & II-B & $\cdots$ & $\cdots$ & 0.98 \\
\hline Mon-000877 & $06411678+0927301$ & $\ldots$ & 616943883 & K4 & 91.4 & II-B & 13.3 & $\cdots$ & 2.64 \\
\hline Mon-000919 & $06411329+0931503$ & $\ldots$ & 616919654 & M4 & 79.9 & I & 2.6 & $\cdots$ & 2.52 \\
\hline Mon-000945* & $06404989+0936494$ & 223977953 & 223977953 & K4 & 66.3 & $\mathrm{I}$ & 16. & 0.67 & 2.97 \\
\hline Mon-000996* & $06404131+0951023$ & 500007315 & 616849542 & K7 & 24.5 & I & 15.5 & 0.61 & 2.60 \\
\hline Mon-001022* & $06403911+0950586$ & 500007252 & 616849543 & $\mathrm{~K} 4$ & 46.5 & III-B & 15. & 0.64 & 5.23 \\
\hline Mon-001174 & $06401370+0956305$ & 400007614 & 616826810 & M2 & 130.3 & III-B & 10.1 & $\cdots$ & 5.59 \\
\hline Mon-001187 & $06401417+0934283$ & $\ldots$ & 602083884 & $\cdots$ & 8.0 & $\cdots$ & 15.5 & $\cdots$ & 2.31 \\
\hline Mon-001217 & $06403665+0952032$ & $\ldots$ & 616849540 & $\mathrm{~K} 4$ & 87.0 & III-B & $\cdots$ & $\cdots$ & 6.88 \\
\hline Mon-001573 & $06401258+1005404$ & 223968439 & 223968439 & $\ldots$ & 26.0 & I & $\cdots$ & $\cdots$ & 2.53 \\
\hline
\end{tabular}

Notes.

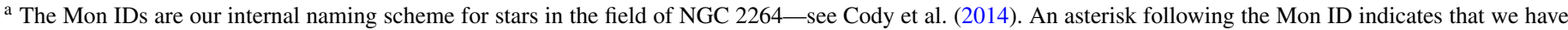
VLT FLAMES spectra for this star from 2011 December.

b CoRoT identification numbers. These uniquely identify the light curve for each CoRoT short run.

${ }^{c}$ See Cody et al. (2014) for the sources of the spectral type and $\mathrm{H} \alpha$ equivalent width (EW) data. All of these are in emission.

'Data from MMT spectra except for Mon 510, 945, and 1022, which are from the VLT/FLAMES spectra.

e Veiling estimate at a wavelength of $6600 \AA ̊$ derived from the VLT spectra.

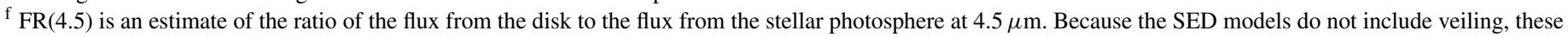
values are likely lower limits.

Table 2

Photometric Data for the Stars of Table 1

\begin{tabular}{|c|c|c|c|c|c|c|c|c|c|c|c|c|}
\hline Mon ID & $u$ & $g$ & $r$ & $i$ & $J$ & $H$ & $K_{s}$ & [3.6] & [4.5] & [5.8] & [8.0] & [24] \\
\hline Mon-000007 & 15.850 & 14.807 & 14.674 & 14.192 & 12.220 & 11.529 & 11.175 & 10.136 & 9.785 & 9.340 & 8.692 & 6.17 \\
\hline Mon-000011 & 18.548 & 16.808 & 15.531 & 14.535 & 12.833 & 12.045 & 11.552 & 10.486 & 10.017 & 9.649 & 8.852 & 5.541 \\
\hline Mon-000117 & 18.562 & 18.326 & 16.832 & 16.358 & 14.162 & 13.430 & 13.070 & 12.469 & 12.136 & 12.038 & $\cdots$ & $\ldots$ \\
\hline Mon-000185 & 17.175 & 15.647 & 14.593 & 14.198 & 12.875 & 12.112 & 11.723 & 10.861 & 10.581 & 10.347 & 9.775 & 6.22 \\
\hline Mon-000260 & 19.983 & 18.550 & 16.956 & 15.905 & 13.358 & 12.232 & 11.498 & 10.402 & 9.915 & 9.491 & 8.707 & 5.422 \\
\hline Mon-000341 & 17.927 & 17.147 & 15.744 & 14.951 & 13.101 & 12.279 & 11.800 & 10.971 & 10.507 & 9.965 & 8.772 & 5.248 \\
\hline Mon-000406 & 17.151 & 16.193 & 15.229 & 14.613 & 13.188 & 12.407 & 12.084 & 11.302 & 10.952 & 10.457 & 9.586 & 6.503 \\
\hline Mon-000412 & 17.872 & 16.624 & 15.461 & 14.699 & 12.770 & 12.002 & 11.694 & 11.063 & 10.720 & 10.333 & 9.726 & 6.692 \\
\hline Mon-000469 & 18.291 & 18.154 & 16.692 & 15.353 & 13.121 & 12.248 & 11.452 & 10.673 & 10.207 & 9.739 & 9.005 & 5.877 \\
\hline Mon-000474 & $\ldots$ & $\ldots$ & $\ldots$ & $\ldots$ & 10.434 & 9.533 & 8.858 & 8.160 & 7.621 & 7.210 & 6.496 & 3.49 \\
\hline Mon-000510 & 16.568 & 15.699 & 14.952 & 14.614 & 12.719 & 11.934 & 11.458 & 10.737 & 10.276 & 9.830 & 9.016 & 5.579 \\
\hline Mon-000567 & 19.064 & 17.601 & 15.995 & 15.254 & 12.775 & 11.786 & 11.015 & 10.087 & 9.616 & 9.259 & 8.579 & 4.771 \\
\hline Mon-000808 & 18.098 & 16.360 & 15.213 & 14.758 & 12.993 & 12.203 & 11.786 & 11.274 & 11.081 & 10.854 & 10.297 & 6.295 \\
\hline Mon- 000860 & 19.434 & 18.609 & 16.978 & 16.350 & 14.328 & 13.640 & 13.405 & 13.293 & 13.190 & 13.103 & $\ldots$ & 7.403 \\
\hline Mon-000877 & 17.259 & 15.571 & 14.511 & 14.095 & 12.645 & 11.877 & 11.502 & 11.081 & 10.796 & 10.629 & 10.130 & 6.75 \\
\hline Mon-000919 & 20.545 & 18.485 & 16.947 & 15.398 & 12.738 & 12.034 & 11.683 & 11.005 & 10.617 & 10.139 & 9.375 & 6.503 \\
\hline Mon-000945 & 17.364 & 16.233 & 15.082 & 14.443 & 12.399 & 11.611 & 11.160 & 10.516 & 10.157 & 9.887 & 9.257 & 6.242 \\
\hline Mon-000996 & 17.496 & 16.382 & 15.214 & 14.616 & 12.806 & 12.024 & 11.713 & 10.905 & 10.554 & 10.152 & 9.432 & 7.02 \\
\hline Mon-001022 & 17.545 & 16.299 & 15.049 & 14.392 & 12.293 & 11.251 & 10.508 & 9.614 & 9.170 & 8.866 & 8.308 & 5.635 \\
\hline Mon-001174 & 17.485 & 16.967 & 15.727 & 15.098 & 12.920 & 12.131 & 11.584 & 10.605 & 9.985 & 9.270 & 8.162 & 5.378 \\
\hline Mon-001187 & 21.403 & 18.943 & 17.510 & 15.997 & 13.753 & 12.964 & 12.587 & 11.869 & 11.518 & 11.230 & 10.535 & 7.261 \\
\hline Mon-001217 & 17.939 & 16.900 & 15.573 & 14.806 & 12.733 & 11.759 & 11.101 & 10.022 & 9.401 & 8.926 & 7.923 & 4.641 \\
\hline Mon-001573 & 18.151 & 16.481 & 15.298 & 14.593 & 12.511 & 11.792 & 11.404 & 10.681 & 10.446 & 9.921 & 9.483 & 6.665 \\
\hline
\end{tabular}

Notes. Broadband photometry for the stars from Table 1, all in units of magnitudes, though a mix of Vega and AB magnitudes. The ugri data are from CFHT, are reported in L. Venuti et al. (2014, in preparation), and are in AB magnitudes; the $J H K_{s}$ data are Vega magnitudes from the online 2MASS all-sky point source catalog; the IRAC and MIPS data are Vega magnitudes from Sung et al. (2009), based on Spitzer imaging obtained in 2004. 

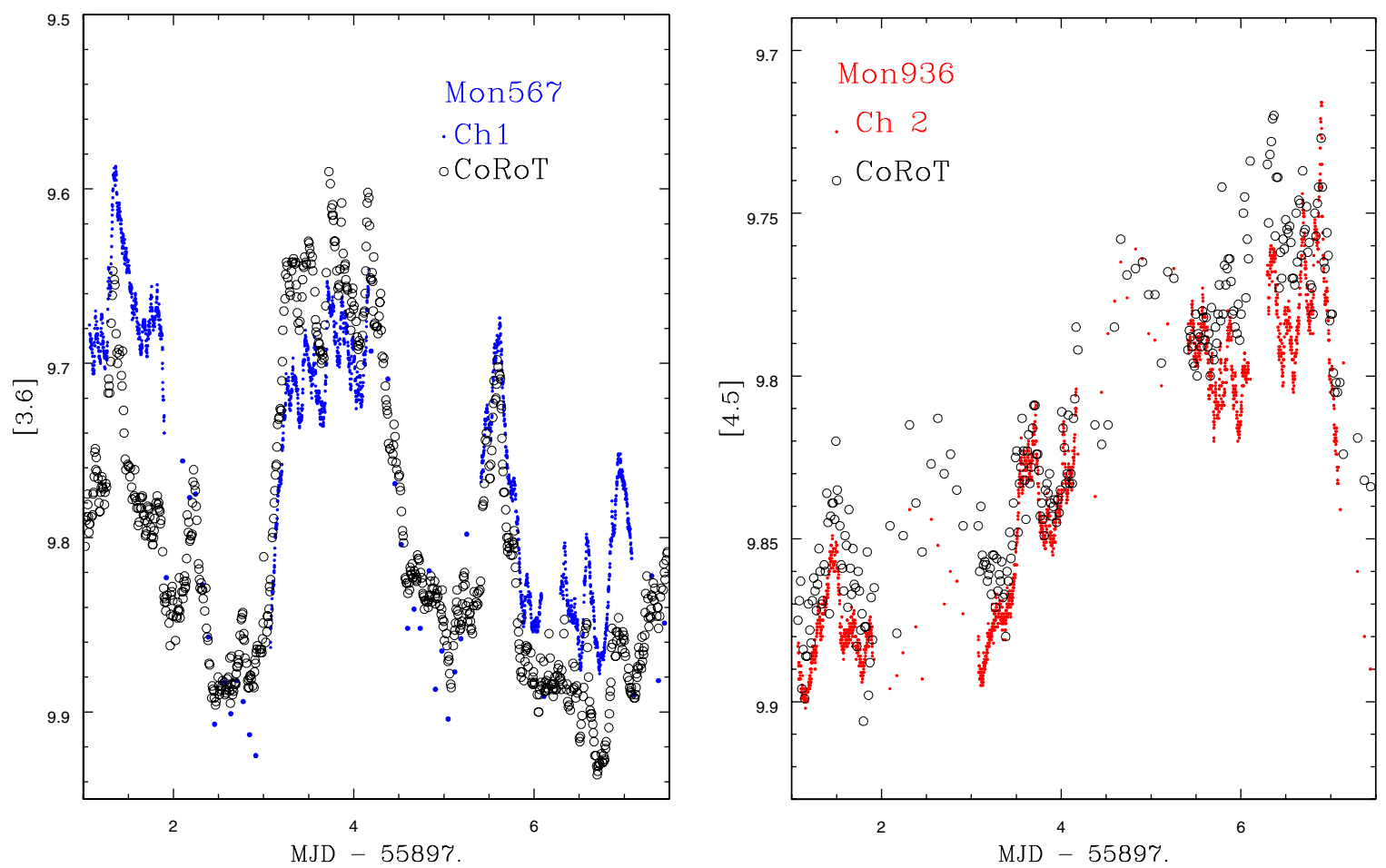

Figure 2. Spitzer and CoRoT data for two stars from Table 1 (Mon-000567, left; and Mon-000936, right) where we have IRAC staring-mode (high-cadence) data, illustrating the often very good correlation between the optical and IR light-curve shapes. Ch1 and Ch2 refer, respectively, to IRAC's 3.6 and $4.5 \mu$ m channels. For Mon 936, which is faint in the optical, we have rebinned the CoRoT data to 20 minute sampling, centered on the nearest IRAC data point.

(A color version of this figure is available in the online journal.)
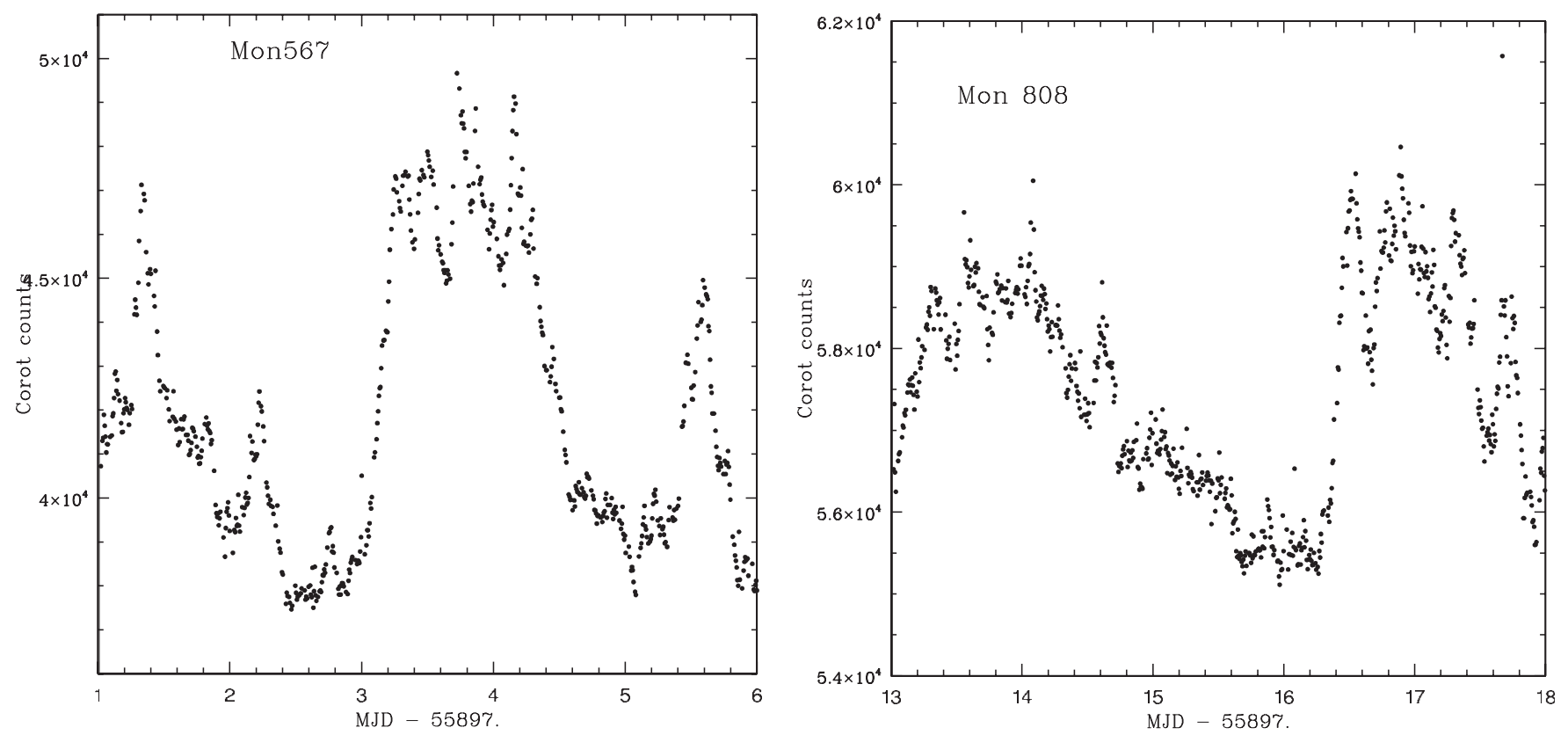

Figure 3. Expanded view of five-day windows for light curves of two of the accretion burst stars from Figure 1, Mon-000567 (left), and Mon-000808 (right). These plots illustrate that when viewed at an expanded scale, the relatively broad (day or a few day) flux excesses often break up into many much shorter duration events.

from the accretion bursts because of their shorter timescales of generally $<1 \mathrm{hr}$ and their characteristic very short rise time and relatively slow decay. Only about a half-dozen likely flares are identifiable in the CoRoT light curves of the stars in Table 1.

\subsection{Long-term Stability of the Accretion Signature}

Ten of the stars in Table 1 have CoRoT light curves from both 2008 and 2011. Based on examination of the light curves, for six of the stars (Mon-000007, 341, 945, 996, 1022, and 1174) we would classify them as members of the accretor class at both epochs. Mon-000185 appears to have a similar character at both epochs, but none of the bursts in 2011 have an amplitude $\geqslant 5 \%$, and therefore we would not classify that light curve as burst-dominated. Two of the stars (Mon-000011 and 510) were identified as accretors based on their 2011 light curves, but have more complicated light curves in 2008. However, each of 

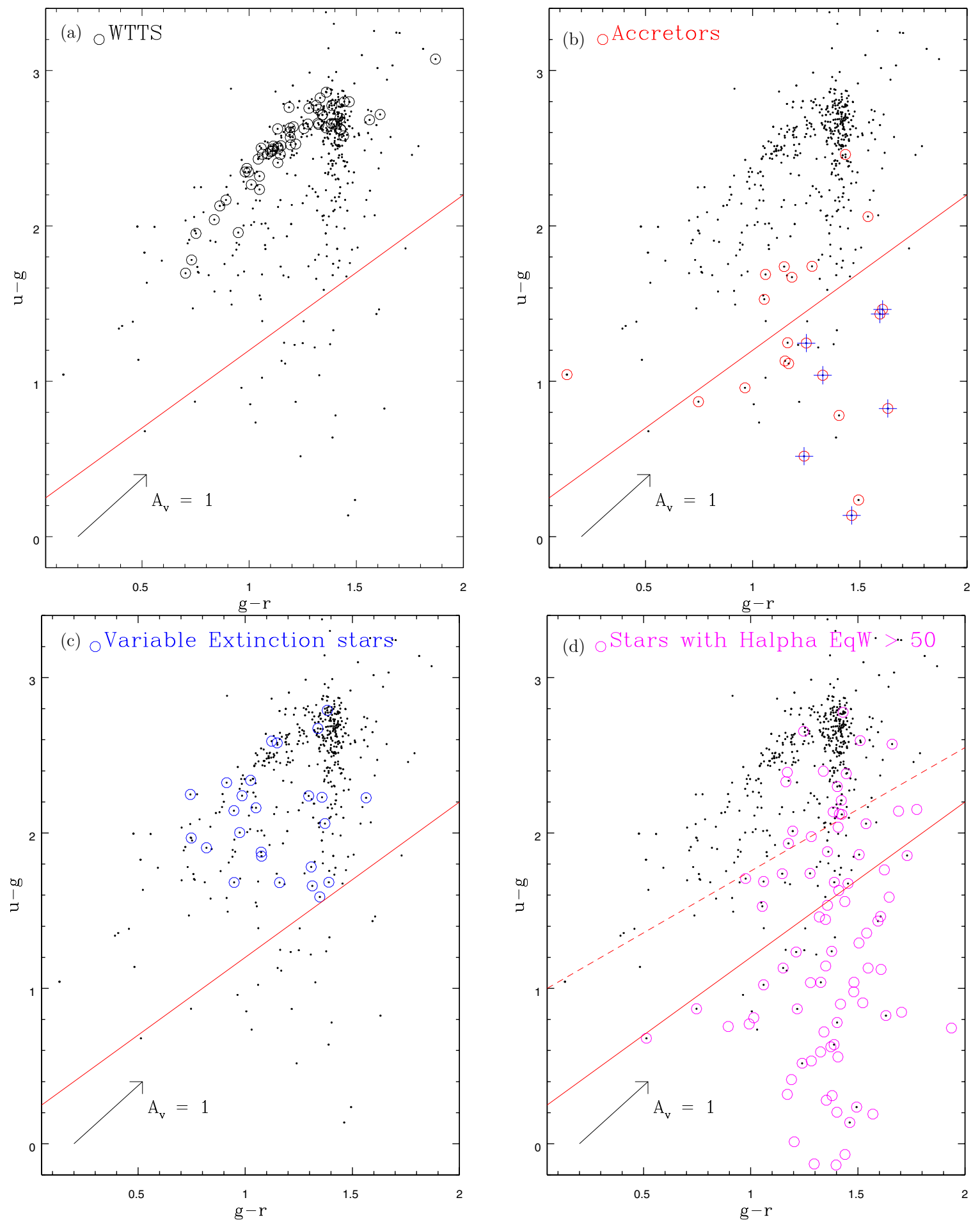

Figure 4. Color-color diagrams derived from CFHT ugri photometry of NGC 2264 (L. Venuti et al. 2014, in preparation). For panels (a)-(c), only stars with CoRoT light curves are plotted. For panel d, the magenta circles correspond to NGC 2264 members, regardless of whether there is a CoRoT light curve or not. Small black dots correspond to all likely members having CFHT photometry. Panel (a) highlights stars that are probable WTTSs without warm circumstellar dust or active accretion; panel (b) highlights stars with accretion-burst-dominated light curves (accretors whose $\mathrm{H} \alpha$ profiles are of Reipurth type III-B are further marked with a blue plus sign; see Section 4.2); panel (c) highlights the variable extinction stars-stars with flux dips, sometimes periodic, thought to be due to portions of the disk passing through our line of sight, causing enhanced extinction; and panel (d) highlights all NGC 2264 members with H $\alpha$ equivalent widths $>50 \AA$. The plots indicate that the accretion burst class members have large UV excesses, while the variable extinction stars have a range of UV colors, from nearly photospheric to mild UV excess. The red solid line provides a plausible boundary for stars with the largest UV excesses-stars below the red line should be the most actively accreting members of NGC 2264. The red dashed line is discussed in Section 7.

(A color version of this figure is available in the online journal.) 

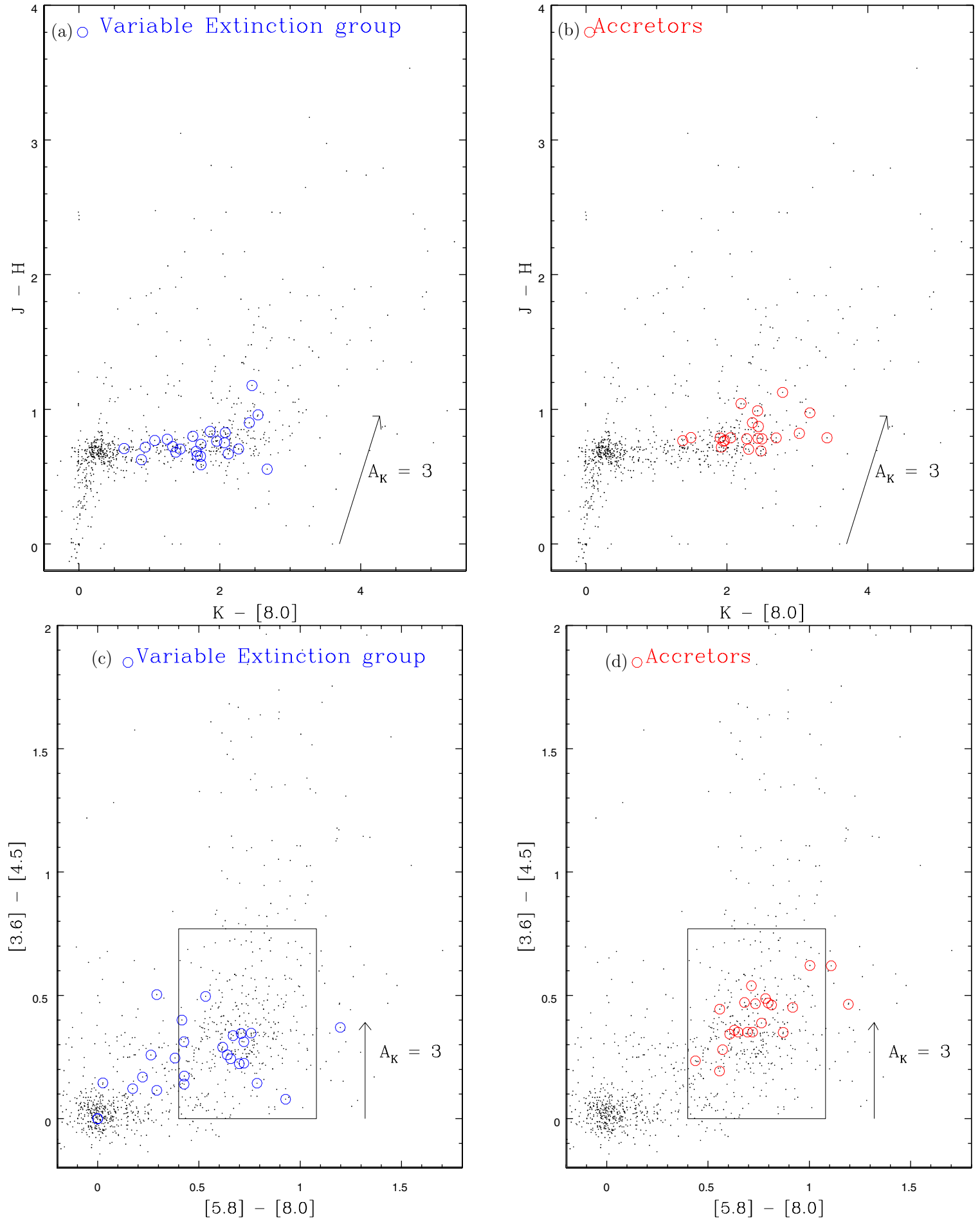

Figure 5. Comparison of the distribution for the variable extinction stars (blue points) and members of the accretion burst class (red points) in two IR color-color diagrams. Panels (a) and (b) are in $J-H$ vs. $K_{s}-[8.0]$; panels (c) and (d) show the same set of stars in [3.6]-[4.5] vs. [5.8]-[8.0] diagrams. The box in panels (c) and (d) corresponds to the locus for Class II YSOs as originally defined by Allen et al. (2004). The small black dots are all NGC 2264 members and candidate members, regardless of whether we have CoRoT light curves or not. The diagrams show that the stars with accretion-burst-dominated light curves have well-developed disks, whereas the variable extinction stars often have weaker IR excesses, with nearly a third falling blueward in [5.8]-[8.0] color, than the Class II box of Allen et al. (2004). (A color version of this figure is available in the online journal.)

those stars has a number of flux bursts in the 2008 data, thereby indicating that the same mechanism dominating the 2011 light curves was also present in 2008. The only star with a much different light-curve morphology at the two epochs is Mon001573, which had an approximately sinusoidal light curve characteristic of a spotted star in 2008 and an accretion-burstdominated light curve in 2011.

\section{CHARACTERISTICS OF THE STOCHASTIC ACCRETOR CLASS-BASED ON BROAD-BAND PHOTOMETRY AND HIGH-RESOLUTION SPECTROSCOPY}

The stars in Table 1 were identified as having unusually active accretion solely on the basis of their light-curve morphology. 

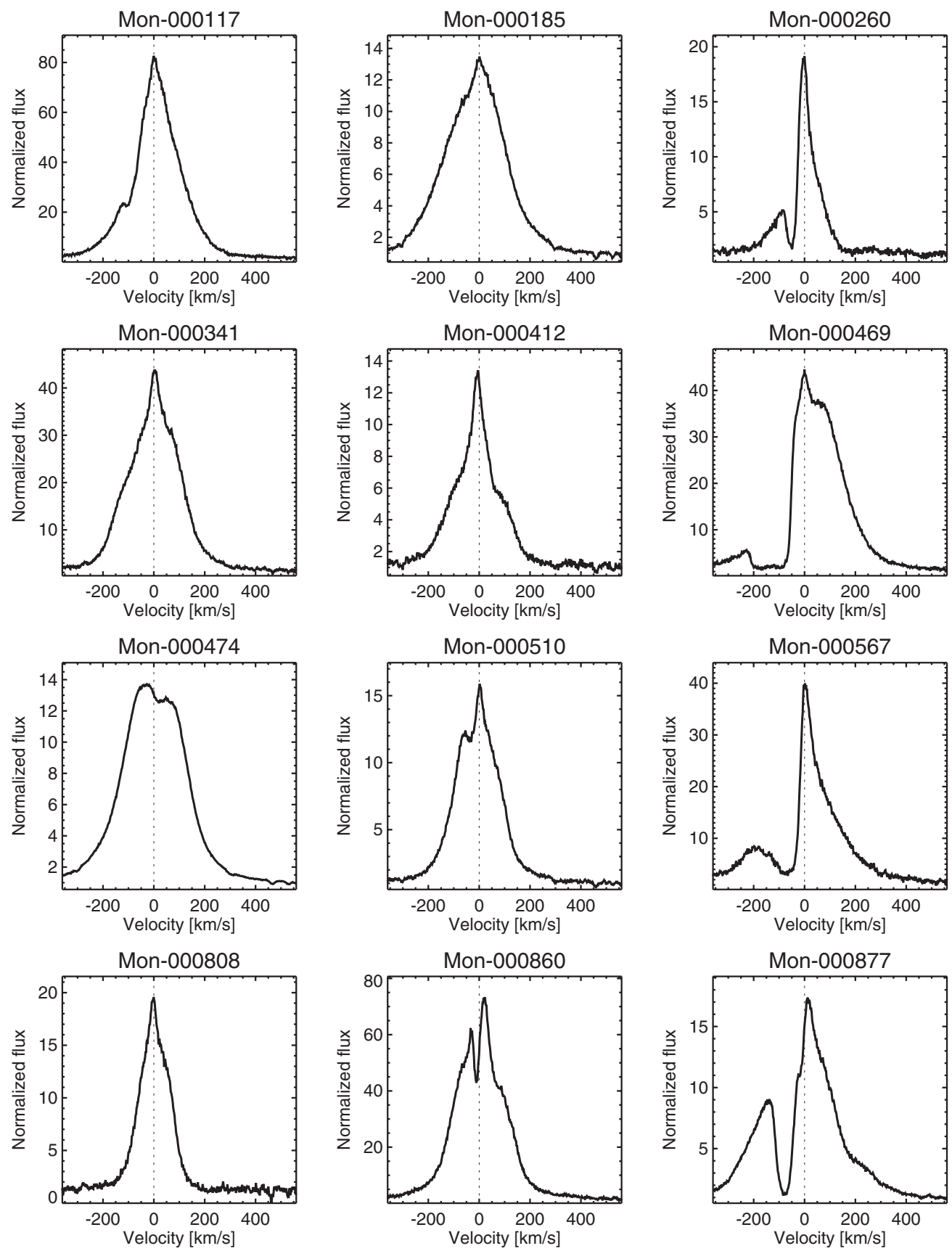

Figure 6. H $\alpha$ profiles of the stars whose CoRoT light curves are categorized as being dominated by accretion bursts, for those stars for which we have available highresolution spectra. The emission profiles have been shifted to the mean cluster velocity, marked by the dashed line at $v=0$.

If that identification is correct, it seems likely that their other observable characteristics, such as IR and UV excesses and Balmer emission line profiles, should also be distinctive. We now show that is indeed the case.

For the remainder of this section, we will compare the YSOs in Table 1 to two other sets of YSOs in NGC 2264. The first of these are 81 WTTSs, identified on the basis of their having stable, sine-wave-shaped, periodic light curves typical of cold spots (Class I light curves of $\mathrm{H} 94$ ), and small $\mathrm{H} \alpha$ equivalent widths (we subsequently verified they also do not have IR excesses). These WTTSs are expected not to have actively accreting circumstellar disks and hence little or no UV excesses. The second group are 26 stars whose CoRoT light curves show periodic or aperiodic flux dips that we believe are best ascribed to variable extinction (Class III light curves of H94). The stars in Figures 1(a)-(c) are members of this variable extinction group. These stars must have disks if our interpretation of their flux dips is valid. The NGC 2264 stars that fall into these two light-curve classes, particularly the variable extinction class, are discussed further in Cody et al. (2014), and in Alencar et al. (2010).

The most certain property that the stars in Table 1 should have if they have unusually active accretion is enhanced UV emission. To determine if that is the case, we use ugri photometry obtained at CFHT. Figure 4 shows the $u-g$ versus $g-r$ color-color diagram for the NGC 2264 YSOs for which we have CoRoT light curves. In panel (a), the WTTSs define the locus of YSOs without UV excesses, with spectral types ranging from mid-G at $u-g \sim 1.7, g-r \sim 0.7$, to early 

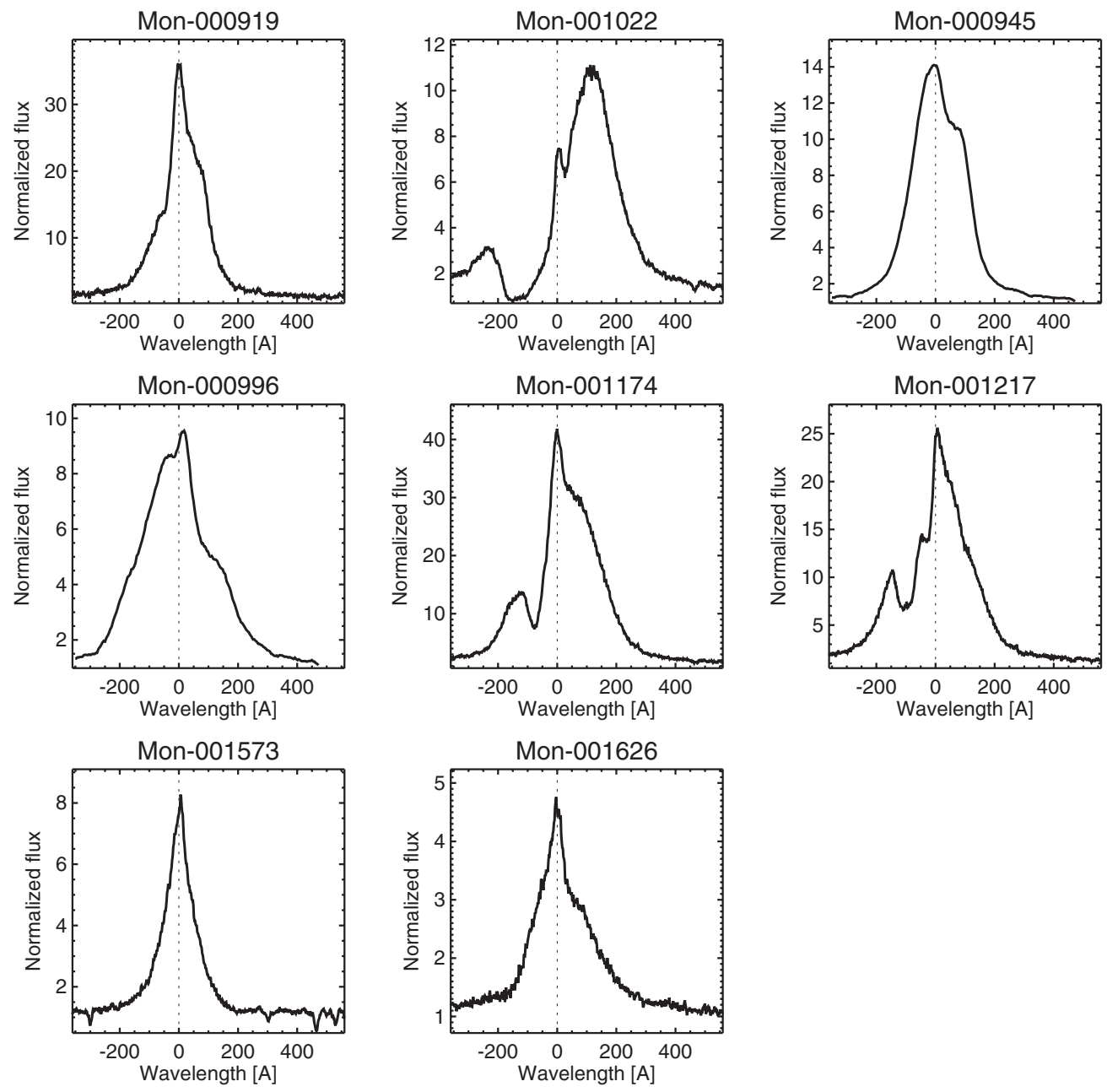

Figure 6. (Continued)

$\mathrm{M}$ at $u-g \sim 2.8$ and, $g-r \sim 1.4$. Panel (b) shows the stars from Table 1, confirming that nearly all of them have UV excesses relative to the WTTS locus, with some of them having very blue $u-g$ colors. Panel (c) shows that the variable extinction stars have intermediate properties - the majority of them have UV excesses, but of comparatively small degree, and a significant number of them have essentially no UV excess. Panel (d) indicates, not unexpectedly, that stars with large $\mathrm{H} \alpha$ equivalent widths also usually have large UV excesses. Among the stars with large $\mathrm{H} \alpha$ equivalent widths and no CoRoT data are likely many more stars with light curves like those in Table 1. Converting the UV excesses to a mass accretion rate following the prescription of L. Venuti et al. (2014, in preparation), the mean accretion rate for the stars of Table 1 is $1.1 \times 10^{-7} M_{\odot} \mathrm{yr}^{-1}$, and that for the variable extinction group of stars is $2.5 \times 10^{-8} M_{\odot} \mathrm{yr}^{-1}$.

The red solid line in Figure 4 serves as an arbitrary but reasonable boundary below which are found the CoRoT stars with the largest UV excesses in NGC 2264. Fourteen of the twenty-six YSOs falling below that line are included in Table 1. The light curves for the other 12 stars are provided in Figure 23. One of these stars (Mon-000273) is only slightly variable, and it is difficult to characterize the features that are present given their low amplitude relative to the noise fluctuations. Another star (Mon-000423) has several jumps or gaps in its light curve, also making it difficult to separate real features from artifacts. Of the remaining 10, five have light curves that could be interpreted as being dominated by short-duration flux bursts (Mon-000766, Mon-000893, Mon-001061, Mon-000168, and Mon-001048); we did not include them in Table 1 because either their continuum level is poorly defined or the noise level is too high. Depending on the interpretation of these five stars, stars with burst-dominated light curves compose at least $55 \%$ and possibly up to $\sim 80 \%$ of the YSOs with large UV excesses and useful CoRoT light curves. Only one of the stars from Figure 23 (Mon-001132) has a light-curve shape that could correspond to a hot spot with lifetime long compared to the rotation period, as predicted for the funnel-flow accretion models of KR08. That burst-dominated light curves would dominate over stable hot spot light curves from funnel-flow accretion at high accretion rates was one of the predictions of KR08; H94 had also found this to be true in their sample of YSOs.

\subsection{Comparison of the Disk Properties of the NGC 2264 CTTSS}

If the stars of Table 1 have accretion-dominated light curves, it must also be true that they should have IR SEDs that indicate the presence of warm dust in their inner circumstellar disks. Figure 5 examines this point using two different IR color-color 

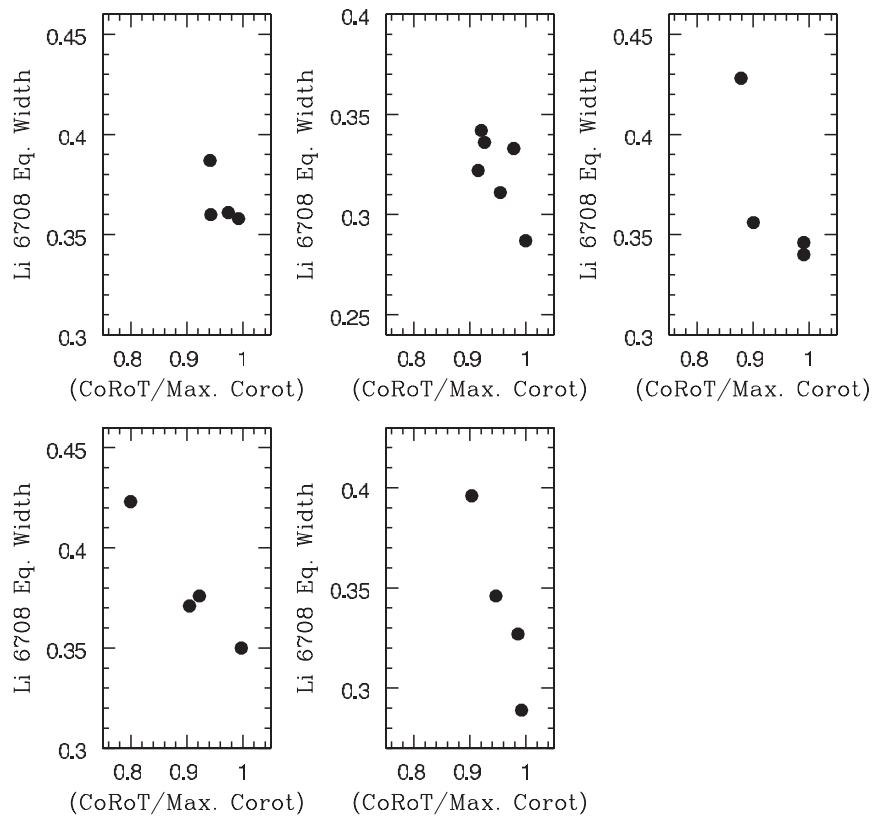

Figure 7. Correlation between the level of veiling (using Li $\lambda 6708$ equivalent as a proxy) and the observed broad-band continuum level from the CoRoT light curves for the five stars with accretion-burst-dominated light curves for which we have VLT/FLAMES spectra. From top-left to bottom-right, the stars plotted are Mon-000341, Mon-000510, Mon-000945, Mon-000996, and Mon-001022.

Lithium equivalent widths are in angstroms; the abscissa points are the CoRoT count rate at the epoch of the VLT spectrum divided by the maximum CoRoT count rate during the time window encompassed by the VLT observations. Based on similar spectra for three WTTSs of similar brightness, the uncertainties in the individual lithium equivalents are of order $0.011 \AA$.
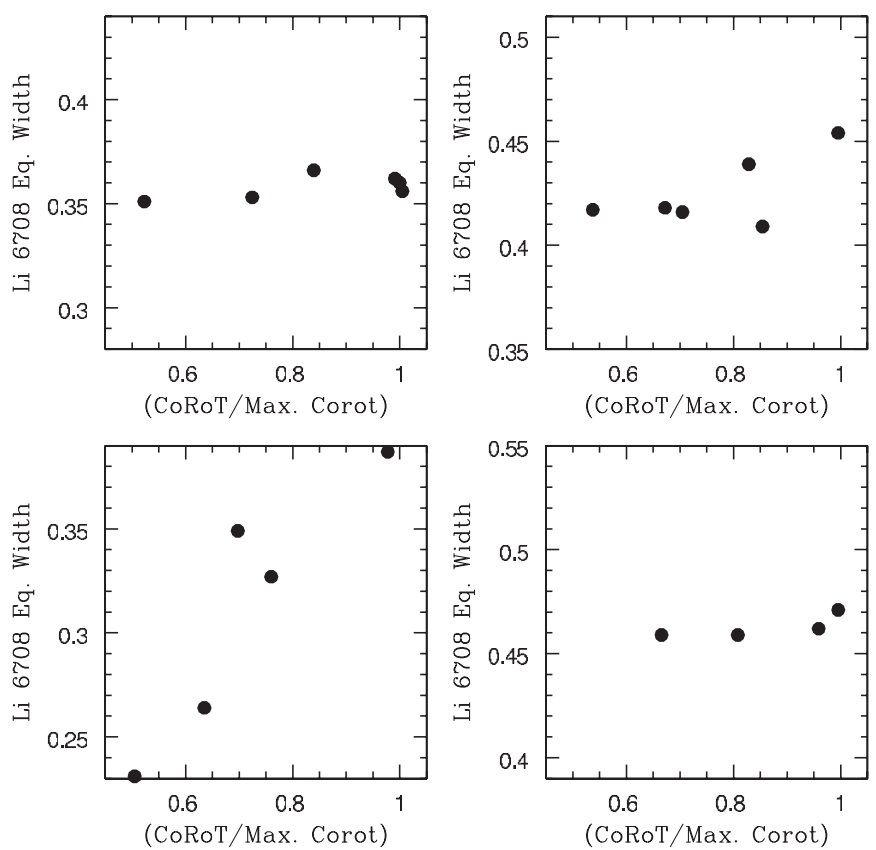

Figure 8. As for Figure 7, except plotting data for the four stars from our variable extinction group where we have sufficient data. From top-left to bottom-right, the stars plotted are Mon-000297, Mon-000660, Mon-001144, and Mon-001199.

diagrams; the top two panels show the variable extinction and accretion burst stars in a $J-H$ versus $K_{s}-[8.0]$ diagram, while the bottom two panels show the same set of stars in a [3.6]-[4.5] versus [5.8]-[8.0] diagram. Teixeira et al. (2012) have constructed theoretical models to predict the IR colors of

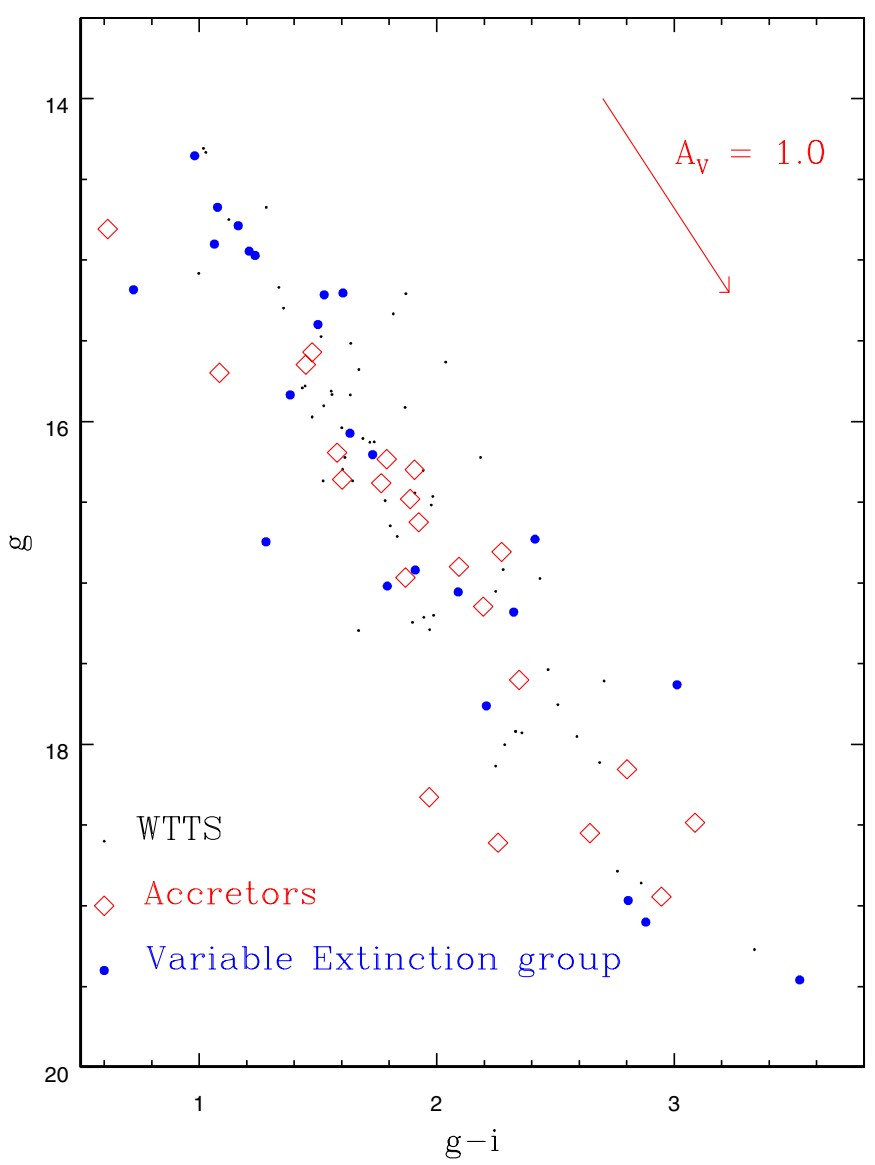

Figure 9. $g$ vs. $g-i$ color-magnitude diagram, for the same set of WTTSs (small black dots), accretion burst stars (red diamonds), and variable extinction group stars(blue dots) as plotted in Figure 4. We see no systematic displacement between the three groups shown, e.g., all three seem to be the same approximate age, or at least that any systematic age difference is too small for us to determine from this figure.

(A color version of this figure is available in the online journal.)

YSOs in the $J-H$ versus $K_{s}-[8.0]$ diagram, showing that the disk population is expected to form a narrow band of stars stretching to the right (as observed). They find that "anemic" disks are restricted to $K_{s}-[8] \sim 1.3$ in the diagram, and Class I sources to $K_{s}-[8]>3$. Bare photospheres have $K_{s}-[8]<$ 0.5 ; embedded YSOs with large reddening or a disk viewed at large inclination angle fan out to the upper right in this diagram. The IRAC color-color diagram was introduced in Allen et al. (2004) and has been used extensively to separate YSOs by disk class. The box shown in the lower two panels is from Allen et al., and is designed to isolate Class II YSOs. Bare photospheres are located near 0,0 in the diagram, and Class I sources lie redward of the Class II box. In both diagrams, nearly all of the stars of Table 1 have colors corresponding to Class II YSOs. The variable extinction stars have IR colors that are significantly less red on average than the Table 1 stars, with nearly half of them falling blueward of the Class II locus. Several of the variable extinction class members have disk colors consistent with classification as transition or pre-transition disks (Espaillat et al. 2007). Espaillat et al. (2012) showed that the accretion rates of transition and pre-transition disks are lower than for normal Class II YSOs, in accord with our finding that the mean accretion rate of the variable extinction group as estimated from their UV excesses is relatively low. 


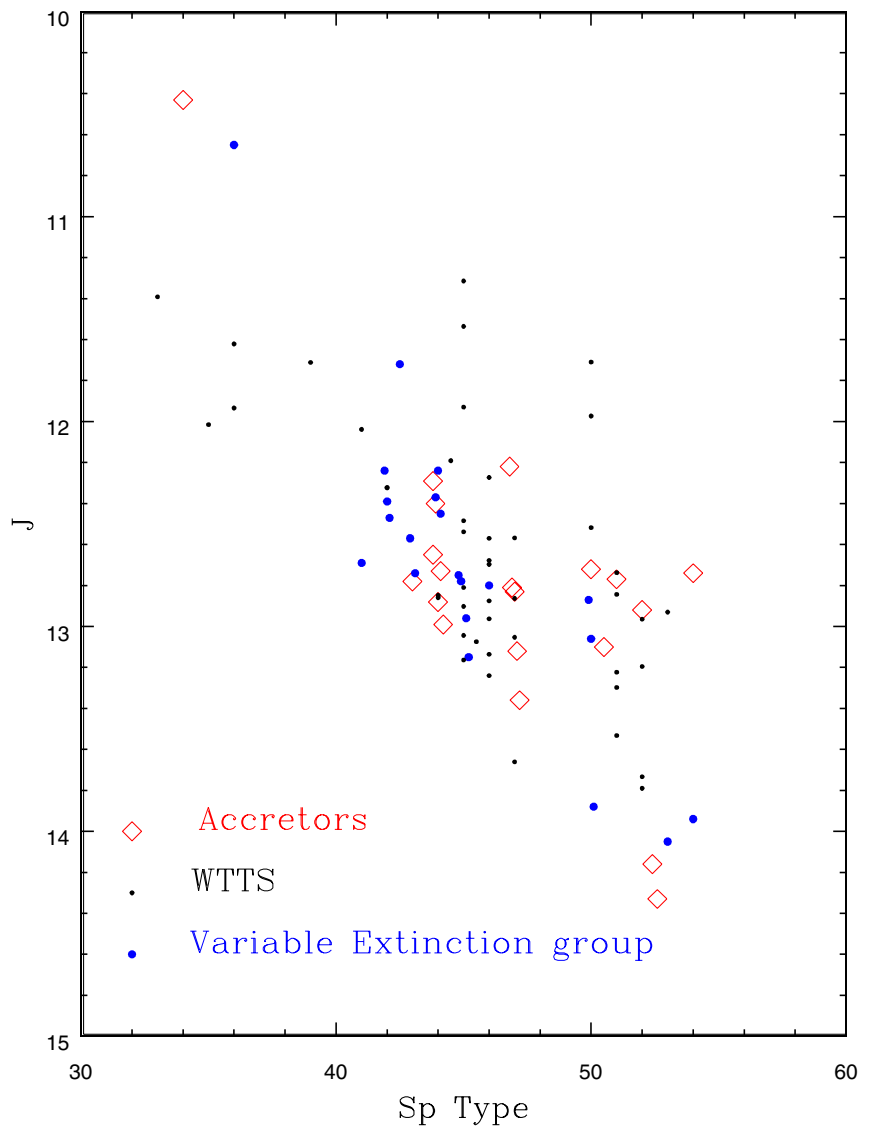

Figure 10. $J$ vs. spectral type diagram, for the same set of stars plotted in Figure 4. As for Figure 4, small, black dots are WTTSs, red diamonds are stars with accretion-burst-dominated light curves, and blue dots are from the variable extinction group. Spectral types have been converted to integer equivalents, with $\mathrm{G} 0=30, \mathrm{~K} 0=40, \mathrm{M} 0=50$, etc. We see no systematic displacement between the three groups shown, e.g., all three seem to be the same approximate age, or at least that any systematic age difference is too small for us to determine from this figure.

(A color version of this figure is available in the online journal.)

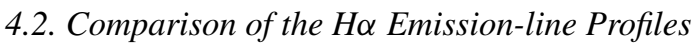 of the NGC 2264 CTTSs}

One of the defining characteristics of CTTSs is that they show strong emission lines in their optical spectra. At high spectral resolution, the shapes of these emission lines are often quite complex, with blueshifted and/or redshifted absorption components and asymmetries (Reipurth et al. 1996, hereafter R96; Alencar \& Basri 2000, hereafter AB00). It is expected that these profile shapes encode information on the kinematics of the gas (infall of accreting gas; outflow of gas from stellar and disk winds) and geometry of the system and our view angle to it. We have $\mathrm{H} \alpha$ profiles for a large fraction of the stars in Table 1, though for most stars the spectra are from 2004/2005. We use these spectra here to help further compare the stars of Table 1 with other CTTSs and thereby to illuminate their physical nature. We also discuss whether the non-simultaneity of the spectra and photometry significantly reduces the utility of the MMT $\mathrm{H} \alpha$ data. Throughout the discussion, we primarily reference the theoretical models of CTTS magnetospheres and disk winds of Kurosawa et al. (2006, hereafter KHS06), but we have also consulted other models (Lima et al. 2010; Kurosawa \& Romanova 2013).
Figure 6 shows the $\mathrm{H} \alpha$ profiles for 20 of the stars in Table 1. In all but two cases, Mon-000945 and 996, the spectra are from the MMT in 2004/2005 (Furesz et al. 2006). The spectral resolution is about 34,000 and the flux scale has been normalized so that the continuum level near $\mathrm{H} \alpha$ has been set to 1.0. The MMT spectra have not been sky subtracted, which can lead to spurious narrow emission features at rest velocity from nebular emission. This is only a significant issue for Mon-000260, where the central peak in Figure 6 may appear much stronger than it really is (see also Figure 24). $\mathrm{H} \alpha$ equivalent widths from the literature for these stars are reported in Table 1.

We first address the issue of the non-simultaneity of the MMT spectra and the CoRoT light curves. In Figure 24 of the Appendix, for the five stars where we have both MMT and VLT FLAMES spectra, we compare the 2004/2005 H $\alpha$ profiles to the ensemble of profiles from the VLT (the dashed lines are individual nightly spectra; the solid line is the average of all VLT epochs). For four of the five stars, the VLT $\mathrm{H} \alpha$ profiles are relatively stable during the 2011 campaign, and also are consistent with the shape of the profile as observed in 2004/2005. Only for Mon-000260 is the MMT profile outside the range of the profile shapes shown in 2011, but even then the difference is relatively small; the ratio of the height of the blue peak to that of the red peak is significantly less than 0.5 for the MMT profile but significantly more than 0.5 for the VLT profiles. This may reflect a real change, or as noted previously, it could be due to nebular emission affecting the MMT red peak. For a qualitative discussion, we therefore believe that use of the 2004/2005 MMT data should be adequate for our purposes.

The H $\alpha$ profiles of Figure 6 are typical of CTTSs, but only sample a relatively small fraction of the total range of profile types shown by CTTSs (e.g., R96 or AB00). Most of the profiles can best be described as lumpy, broad, and centrally peaked, corresponding to Type I profiles of the R96 scheme. Nearly all of the other stars have blueshifted absorption features, with profile types of either II-B (B/R peak ratio between 0.5 and 1$)$ or III-B (B/R peak ratio less than 0.5$)$. To illustrate the relatively small range of profile types among the Table 1 stars, Figure 25 provides the $\mathrm{H} \alpha$ profiles of the stars with transient extinction dips in their light curves as previously discussed in relation to Figures 4 and 5. Many of these profiles are much more complex than the Table 1 stars, with a third having redshifted absorption features (profile type II-R and III-R) and none having the deep, highly blueshifted absorption features normally ascribed to disk or stellar winds (as seen particularly for Mon-000469, 567, and 1022 for the accretors).

KHS06 explicitly compared their model profiles to the R96 $\mathrm{H} \alpha$ classification scheme. They concluded that the type III-B profiles must have quite high accretion rates $(\dot{M} \sim$ $10^{-7} M_{\odot} \mathrm{yr}^{-1}$ ) and are most likely for systems seen at relatively low inclination angle. The stars with type III-B profiles are marked with blue plus signs in Figure 4(b), confirming that they indeed all do have quite large UV excesses and hence presumably quite large accretion rates. The type I profiles require lower, but still significant, accretion rates with little constraint on the inclination angle. The type III-R profiles found among some of the stars with variable extinction light curves generally requires lower accretion rates $\left(\dot{M} \sim 10^{-8} M_{\odot} \mathrm{yr}^{-1}\right.$ or less $)$ and very high inclination angles. The high inclination angle requirement is in good agreement with the physical model for producing the flux dips in the AA Tau light curves, and the weak UV and IR excesses for these stars also agrees with the 

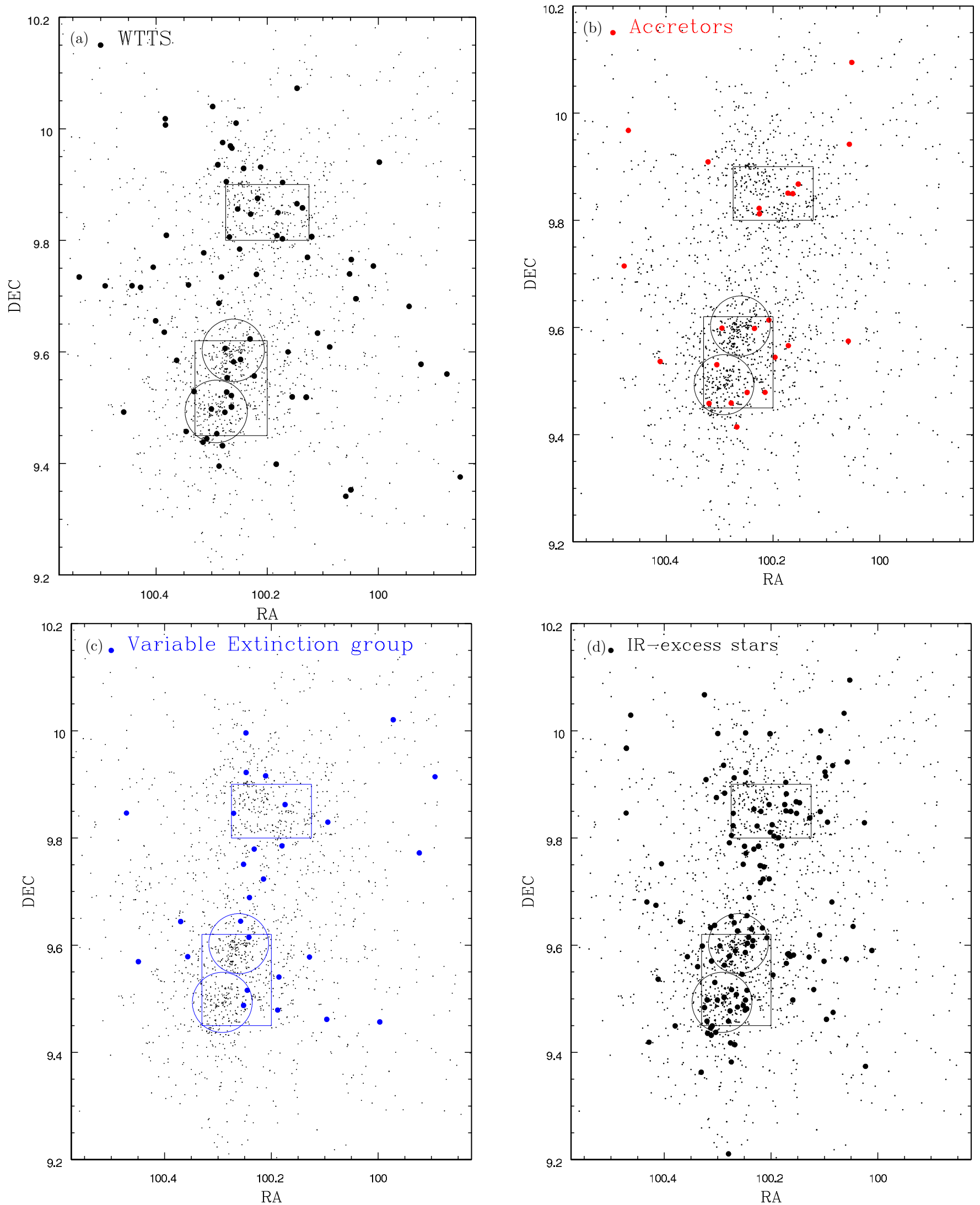

Figure 11. Spatial distribution of YSOs in NGC 2264. The four panels correspond to the same sets of stars as plotted in Figure 4 for the $u-g, g-r$ plots. Panel (a) highlights stars that are probable WTTSs; (b) highlights members of the accretion burst class; (c) highlights stars with extinction dips in their light curves; and (d) highlights all NGC 2264 members classified as Class I or II. The circles and squares enclose the regions of most active current star formation (Sung et al. 2008; L04); see the text in Section 5.2. The plots indicate that the accretion burst class members are more concentrated in the sites of active star formation in NGC 2264 than WTTSs or other types of CTTSs.

(A color version of this figure is available in the online journal.) 


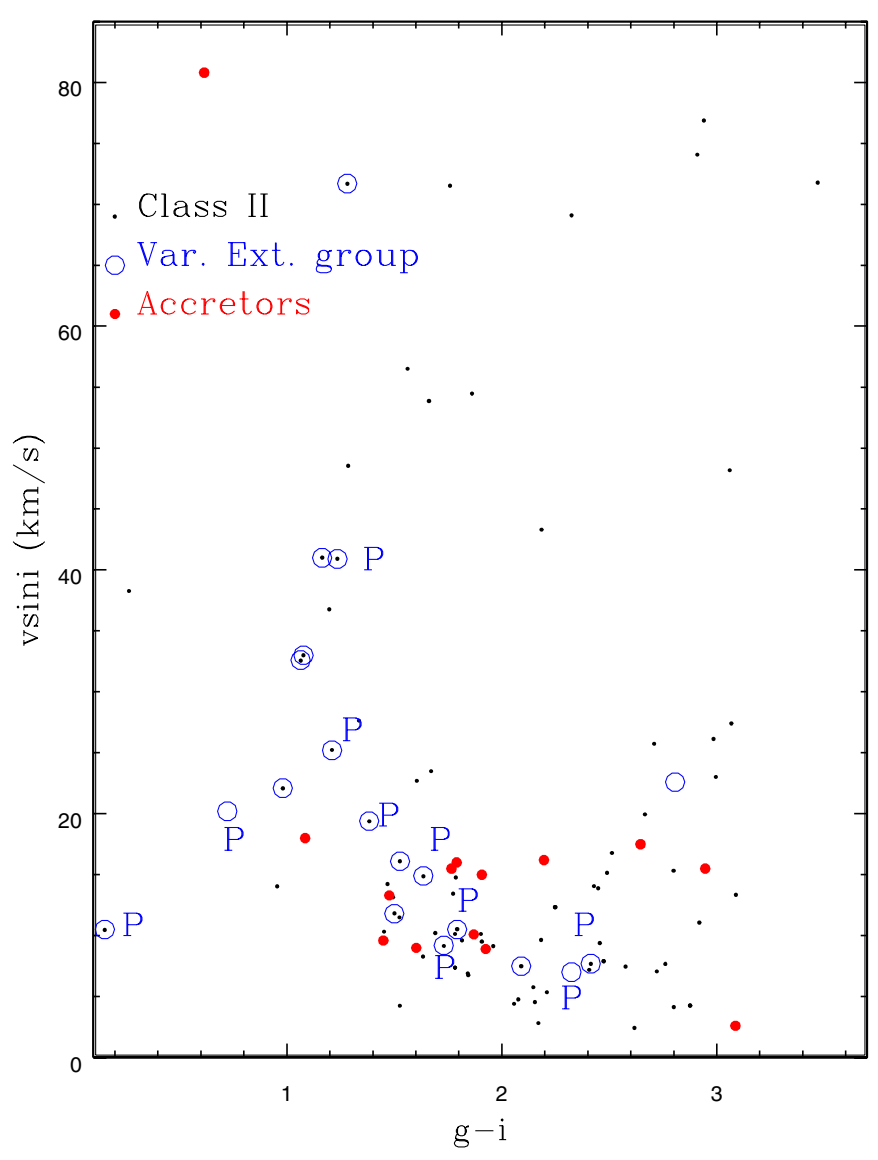

Figure 12. Rotational velocity distribution $(v \sin i)$ as a function of $g-i$ for the stars with accretion-burst-dominated light curves from Table 1, compared to that for the stars of the variable extinction group, and a set of all the CTTSs in NGC 2264 for which we have $v \sin i$ estimates. Black dots are Class II objejcts, open blue circles are objects from the variable extinction group, and filled red dots are objects from the accretor group. Members of the variable extinction group with periodic flux dips are labeled with "P." The variable extinction dip group includes a much larger fraction of relatively early spectral type stars with relatively large rotational velocities.

(A color version of this figure is available in the online journal.)

prediction of a lower accretion rate expected to match their $\mathrm{H} \alpha$ profiles.

The KHS06 model profiles were calculated for stars with stable, funnel-flow accretion, and so conclusions derived from those models do not necessarily apply to our stars, which appear to have more stochastic accretion. However, model Balmerseries emission profiles for stochastic accretors have recently been published by Kurosawa \& Romanova (2013). Based on those models, the $\mathrm{H} \alpha$ profiles for funnel-flow and instabilitydriven accretion appear qualitatively the same, with the primary difference being that the redshifted absorption dips, if present, should be periodic in the former case but aperiodic in the latter case. Because we generally only have a single-epoch $\mathrm{H} \alpha$ profile, we cannot test this prediction for the stars of Table 1.

\subsection{Veiling and Veiling Variability}

In addition to the $\mathrm{H} \alpha$ profiles, the high-resolution spectroscopy we have provides us with an additional means to characterize the stars of Table 1 and compare them to other NGC 2264 CTTSs. In particular, we can measure photospheric veiling and veiling variability (Bertout 1984; Hartmann \&
Kenyon 1990). The strong UV excesses in CTTSs, which we have shown to be especially true for the stars of Table 1, are usually attributed to photons (a combination of black body and free-free/bound-free emission) from the hot gas created when the accreting gas strikes the stellar surface. While the contrast of this emission relative to the photospheres of CTTSs is greatest in the UV, it is still present in the VLT/FLAMES spectral bandpass. The effect is both to add a slowly varying blue continuum to the light from the photosphere, but also in some cases to add emission cores to some of the absorption lines; both effects result in weaker mean absorption line equivalent widths in CTTSs compared to stars with purely photospheric spectra (Petrov et al. 2011). This is normally quantified with a parameter called the veiling factor,

$$
r=(E q W(\text { photosphere }) / E q W(\text { CTTS }))-1 .
$$

In order to provide a mean veiling index for the six stars from Table 1 for which we have VLT spectra, we have coadded all available spectra from the 2011-2012 campaign, corresponding to usually 15 to 22 epochs. We have then measured the equivalent widths for the seven strongest and least blended absorption lines in each of those spectra (at 6448.9, 6461.6, 6470.7, 6624.7, 6707.8, and $6717.7 \AA$-the latter two being $\mathrm{LiI}$ and $\mathrm{CaI}$ ), and done the same for a set of WTTSs of the same spectral type for which we also have VLT spectra. The derived veiling factors at $\lambda \sim 6500 \AA$ for the accretion burst stars are provided in Table 1; based on the scatter in the results for individual absorption features, the typical rms uncertainty in these values is about 0.1 . While significant, the derived veiling factors are not huge - a characteristic value of 0.65 means that the veiling continuum at $6600 \AA$ is $65 \%$ of the photospheric continuum, or equivalently that $39 \%$ of the total continuum at $6600 \AA$ is non-photospheric.

We can use the individual spectra obtained during 2011 December when CoRoT was observing the cluster to look for a correlation between the spectral veiling and the broad-band optical flux. There are usually either four or six epochs where we have both VLT spectra and CoRoT photometry. Because the signal-to-noise ratio of the individual spectra is low, only the Li I $\lambda 6708 \AA$ line can be measured accurately, and we use its equivalent width as a proxy for veiling (though see StoutBatalha et al. 2000 for a cautionary note re: $\mathrm{Li} \lambda 6708$ as a veiling proxy). Figure 7 shows the correlation between our veiling proxy and the CoRoT broad-band photometry for the five stars from Table 1 with such data; Figure 8 shows a corresponding plot for the variable extinction stars where we have sufficient data. The two sets of stars clearly behave differently-the accretion burst group shows larger equivalent widths as the star becomes fainter, whereas the variable extinction set shows either no correlation or larger equivalent widths when brighter. Having larger equivalent widths when fainter is as expected if the driver of the variations is a changing amount of veiling continuum. To first order, variable extinction should have no significant effect on equivalent widths, so the general trends shown in Figure 8 are also as expected. The increase in lithium equivalent width as the star brightens is perhaps unexpected; however, a similar dependence has been seen previously in AA Tau (Bouvier et al. 1999). For AA Tau, the suggested explanation was that the geometry of our line of sight and the warped disk should lead to the enhanced extinction largely affecting the low-latitude portion of the star's photosphere. Hot spots at high latitudes dilute the lithium equivalent widths, and therefore when the star is most extincted (the light is most dominated by light 

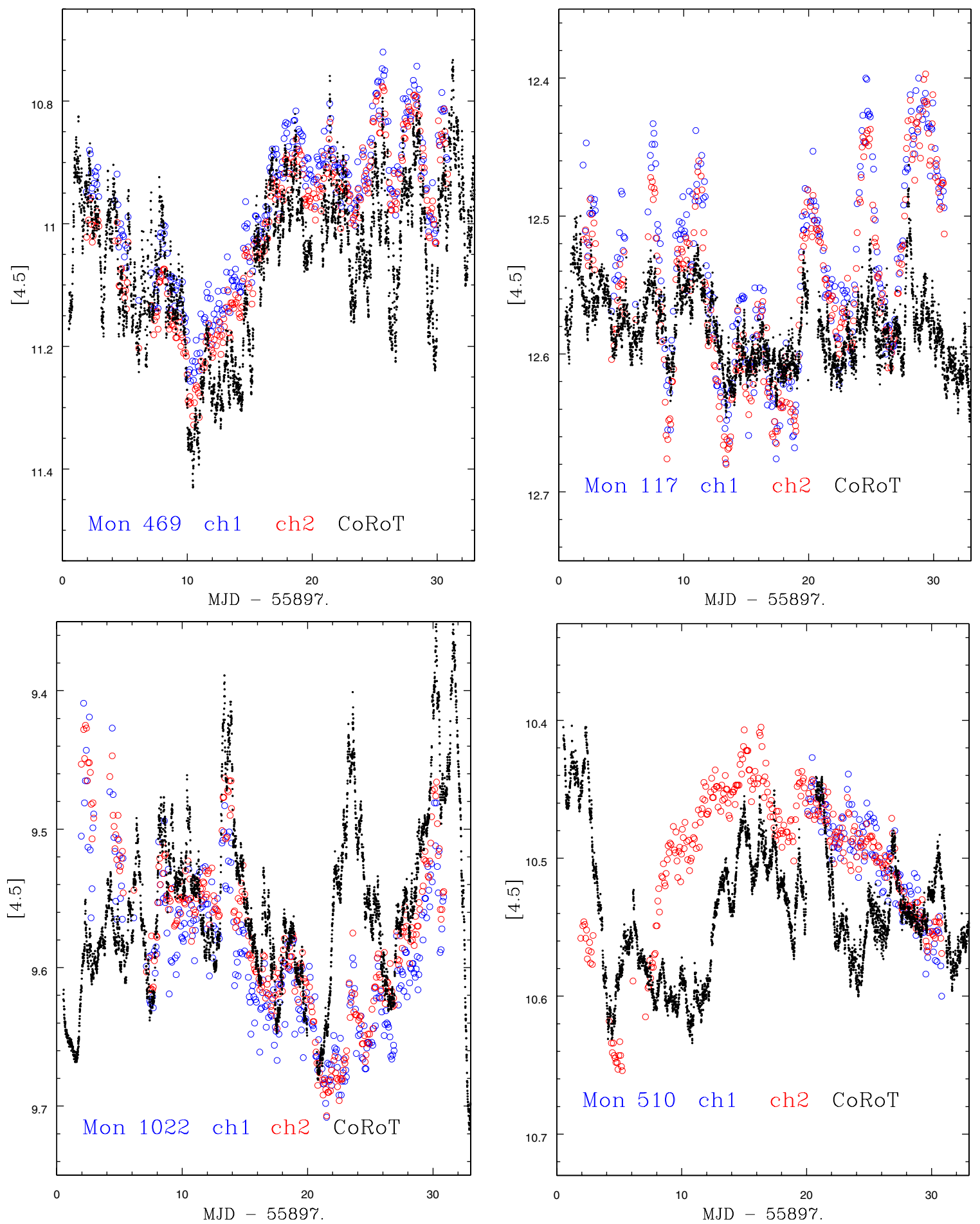

Figure 13. Illustration of the correlation between Spitzer and CoRoT light curves for four of the stars with accretion-burst-dominated light curves. The stars are arranged more or less from most correlated (upper left) to least correlated (lower right).

(A color version of this figure is available in the online journal.)

from high latitudes), the lithium equivalent width should be smallest.

\section{AGE AND VIEW ANGLE INFERENCES FOR MEMBERS OF THE ACCRETION BURST CLASS}

NGC 2264 is normally thought of as slightly older than, for example, the Orion Nebula Cluster (ONC) or Taurus. A commonly quoted average age for the region is about $3 \mathrm{Myr}$ (e.g., Park et al. 2000). However, it is quite clear that there are portions of the star-forming region that are much younger and there is on-going star formation along the molecular ridge extending northward from the Cone Nebula. Some of the YSOs in the youngest parts of NGC 2264 are likely to be $\sim 1 \mathrm{Myr}$ old or younger (Young et al. 2006; Teixeira et al. 2012). An age range from $0.1 \mathrm{Myr}$ to $5 \mathrm{Myr}$ was derived by Dahm \& Simon (2005), with similar estimates by Rebull et al. (2002) and Soderblom et al. (1999). Given that the stars of Table 1 are 

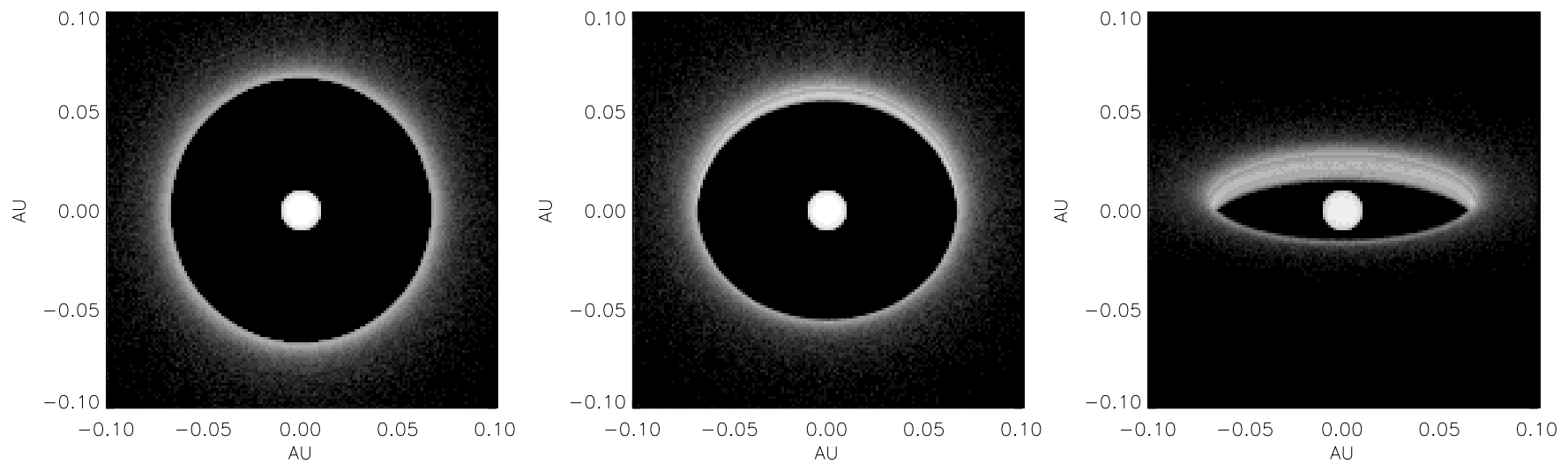

Figure 14. Illustration of the dependence of $4.5 \mu \mathrm{m}$ disk emission on view angle, as predicted from a standard flared-disk model (Whitney et al. 2013). The YSO pictured here has $L$ (photosphere) $=1 L_{\odot}, T_{\text {eff }}=4000 \mathrm{~K}$, and a mass accretion rate of $8 \times 10^{-8} M_{\odot} \mathrm{yr}^{-1}$. The three panels correspond to disk inclinations of $0^{\circ}$, $30^{\circ}$, and $70^{\circ}$. See Section 5.4 for model details.
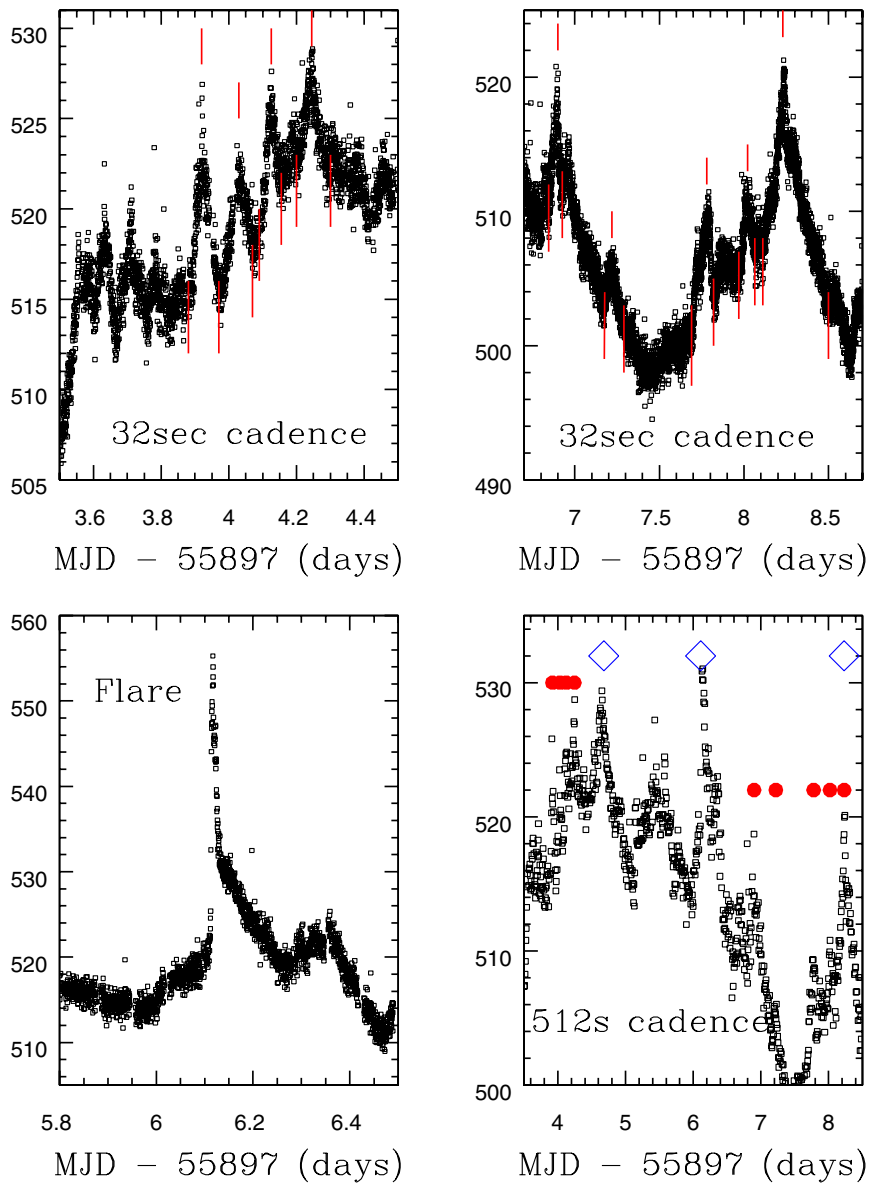

Figure 15. (a, upper left) Expanded portion of the high-cadence Mon-000474 light curve. The four flux bursts we have measured in this segment of the light curve are marked with red bars indicating the beginning, center, and end of the burst; (b, upper right) same as (a) for a different portion of the light curve; (c, lower left) one of two stellar flares identified in the Mon 474 light curve; (d, lower right) A resampled light curve, now at 512 s cadence, for the time period covered in (a) and (b). Red dots mark the location of bursts identified in panels (a) and (b); blue diamonds mark bursts that would be identified at this cadence.

(A color version of this figure is available in the online journal.)

heavily weighted toward high current accretion rates, it seems plausible that they are on average also comparatively young. In this section, we examine to what extent that speculation can be validated by data.
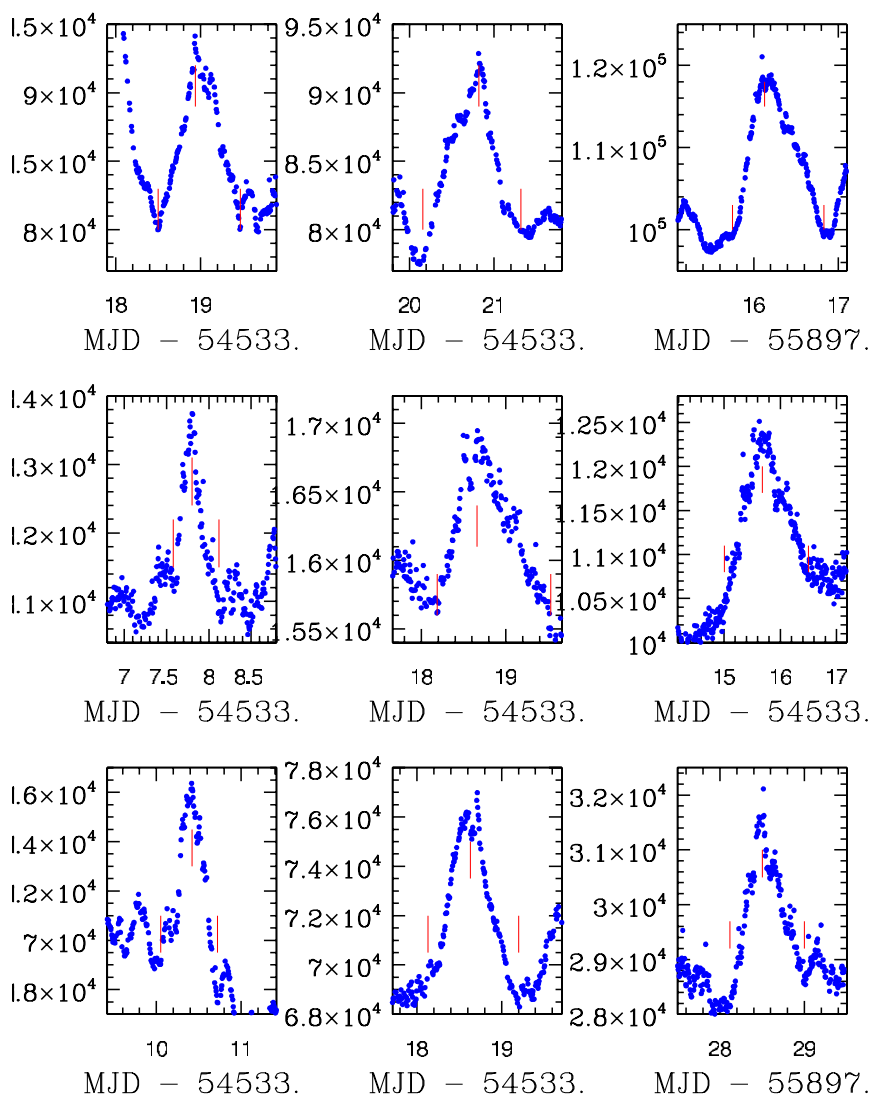

Figure 16. Nine examples of relatively isolated flux bursts in the CoRoT light curves obtained at the normal (512 s sampling) cadence. The red vertical lines mark our estimates of the beginning, mid-point, and end of each burst.

(A color version of this figure is available in the online journal.)

\subsection{Age Inferred from Color-Magnitude and HR Diagrams}

Using our CFHT photometry, Figure 9 compares the location of the stars in Table 1 to the stars with extinction dips and to the WTTSs of NGC 2264 in a $g$ versus $g-i$ color-magnitude diagram (CMD). If one of these sets of stars were younger on average than the others, we would expect the stars of that set to be displaced systematically above the other groups. We see no such systematic displacement between the three groups in the diagram; all three follow essentially the same locus in the CMD. The only possible difference appears to be that the WTTSs may have a smaller dispersion about that locus than 

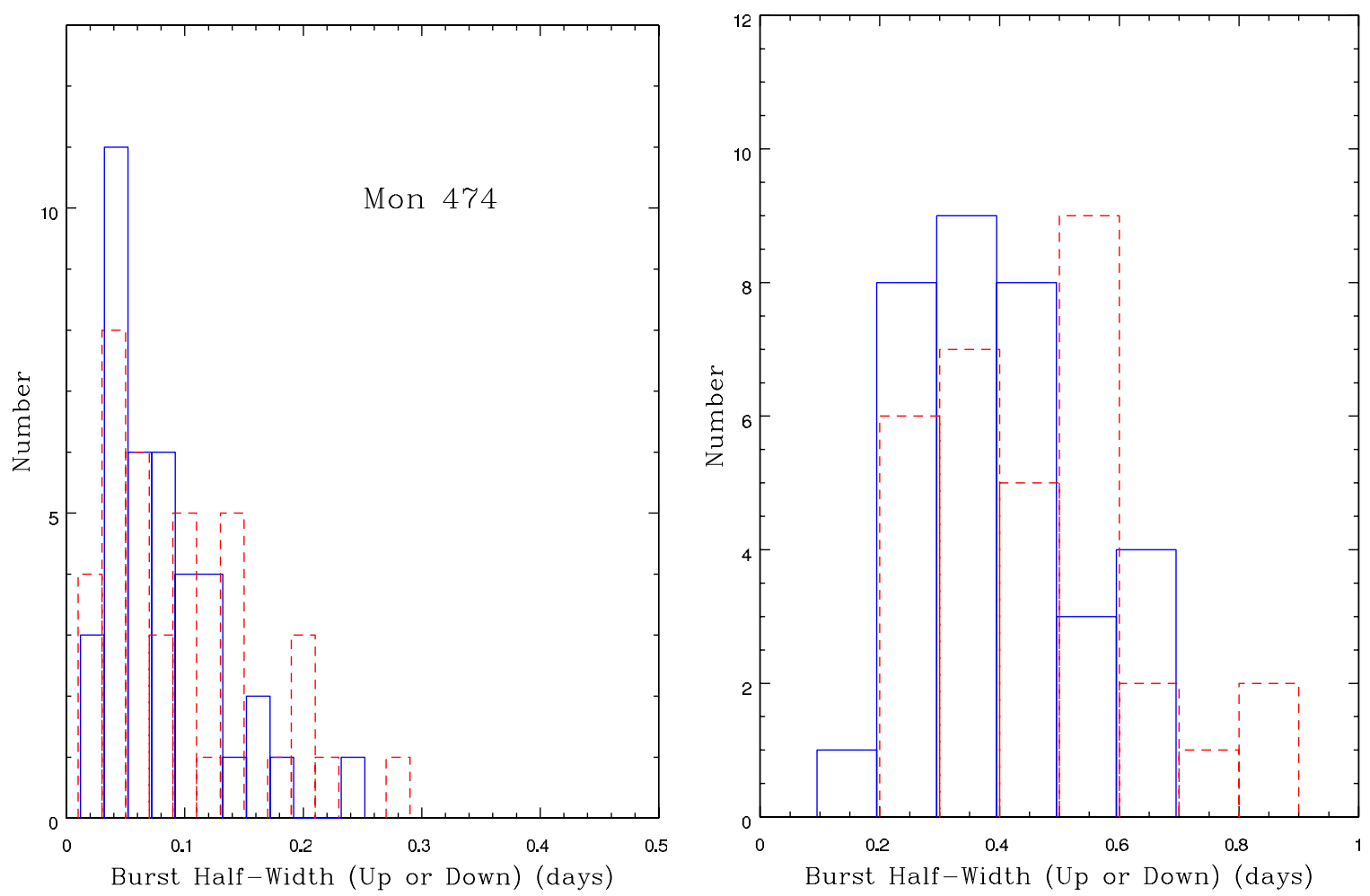

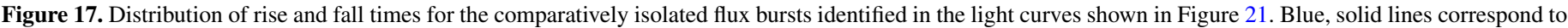

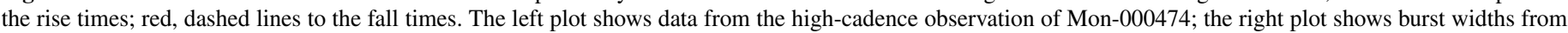

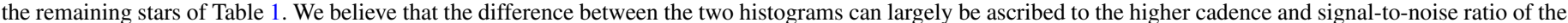

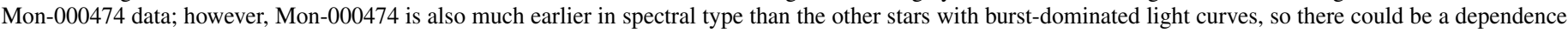
on some mass-related characteristic (e.g., outer convective envelope depth).

(A color version of this figure is available in the online journal.)

either of the two sets of CTTSs (similar results were found by L04). One could argue that in both axes of this plot, the location of the stars with accretion-burst-dominated light curves in particular would be affected by their hot spots, plausibly causing the added dispersion and possibly causing a systematic displacement relative to the other stars. A diagram with perhaps less sensitivity to accretion luminosity is $J$ versus spectral type, which we provide as Figure 10. However, the basic result is the same-the three sets of stars appear to follow the same locus, suggesting no significant age difference or at least that any systematic age difference is too small for us to determine with these proxies for stellar luminosity and effective temperature.

\subsection{Age Inferred from Spatial Location}

As emphasized by Sung et al. (2009) and Teixeira et al. (2012), the regions of current active star formation occupy a relatively small fraction of the total area on the sky for the entire population of cluster members. If, for example, light curves of the type shown in Figures 1(d)-(f) were to occur primarily among the youngest YSOs, then one should find a larger fraction of such stars in the currently star-forming cores. Figure 11 shows the spatial locations of the accretion burst stars, variable extinction stars, and WTTSs from Figure 4, plus (in panel (d)) the set of stars whose SEDs indicate they are Class I or II. The circled regions are the most active star-forming regions from Sung et al. (2008), while the boxed regions are the most active starforming regions from L04. Table 3 provides the fraction of stars in each group that fall within the active star-forming cores, and provides circumstantial evidence that the stars with accretion- burst- dominated light curves are indeed younger, on average, than the other three groups.

\subsection{Rotational Velocity Distribution}

In principle, the spectroscopic rotational velocities $(v \sin i)$ for a group of young stars should depend on the ages and initial angular momenta of the stars, the degree to which their surface rotation is coupled to their circumstellar disks, and the orientation of their rotation axes to our line of sight. Based on our previous discussion, it is likely that the stars of Table 1 are comparatively young and that our line of sight to their rotational axes may be weighted toward small inclinations - both of which would result in relatively small projected rotational velocities. We have $v \sin i$ data for more than half of the stars with CoRoT light curves, so we may be able to check this prediction. Figure 12 compares the rotational velocity distributions for the same groups of stars considered in Figure 11, for the subset of stars for which we have high-resolution spectra.

The primary difference in Figure 12 between the stars with accretion-burst-dominated light curves and the stars with extinction dips, to our eyes, is that the variable extinction dip group includes a larger fraction of comparatively blue stars with relatively large rotational velocities. Only one of the stars in Table 1 seems to be in this extension to high rotational velocities at higher (inferred) masses, and that is Mon-000007 at $g-i=$ $0.6, v \sin i=80 \mathrm{~km} \mathrm{~s}^{-1}$. This difference in colors is not, for example, due to a difference in extinctions. The variable extinction group includes a larger fraction of stars with relatively early spectral types - half of the extinction dip group members with spectral types and $v \sin i$ values have spectral types earlier 

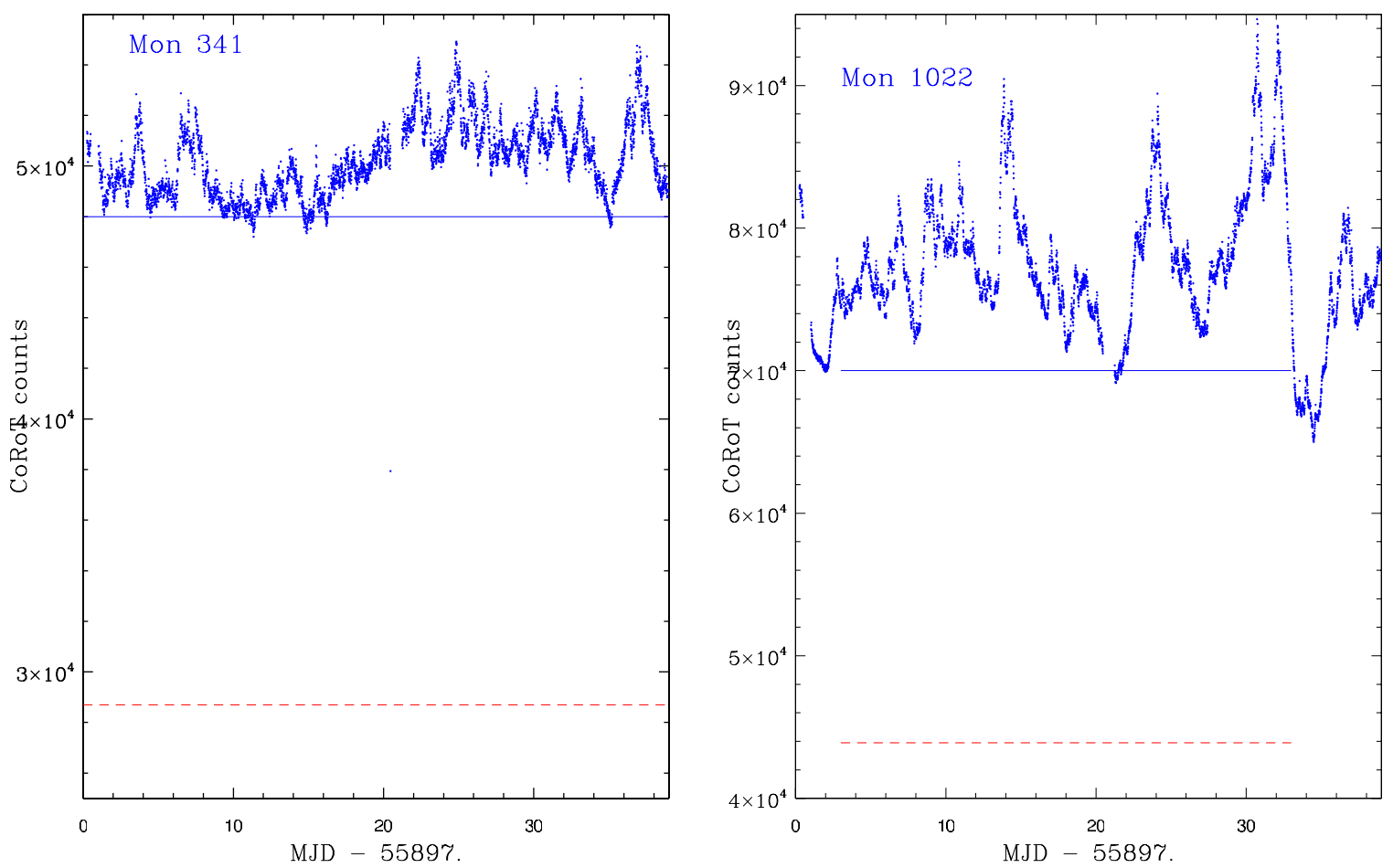

Figure 18. Illustration of the method used to estimate the fraction of the star's broad-band optical light possibly coming from accretion bursts, for two of the stars with VLT/FLAMES spectra. The solid horizontal line is our estimate of the photospheric continuum level. The red dashed line is the photospheric continuum level derived from the estimated veiling factor.

(A color version of this figure is available in the online journal.)

Table 3

Spatial Distribution of YSOs by Type

\begin{tabular}{lccccc}
\hline \hline Group & Total Number & $N$ (Sung) & Fraction (Sung) & $N$ (Lamm) & Fraction (Lamm) \\
\hline Accretion burst stars & 23 & 7 & 0.30 & 13 & 0.56 \\
Extinction dip stars & 27 & 4 & 0.15 & 5 & 0.19 \\
WTTS & 81 & 13 & 0.16 & 20 & 0.25 \\
Class II & 140 & 33 & 0.24 & 48 & 0.34 \\
\hline
\end{tabular}

Notes. "Sung" and "Lamm" refer to the circled and boxed regions of the most active current star formation as shown in Figure 11, as originally defined in Sung et al. 2008 and L04, respectively. $N$ (Sung) is the number of stars of a given group that fall within the circular regions defined by Sung; Fraction(Sung) is the ratio of $N$ (Sung) to the total number of members in that group.

than K4, compared to only one of 15 such stars from Table 1 . We believe that this dependence of the projected rotational velocity on spectral type reflects the initial conditions that lead to the Kraft main-sequence dependence of mean rotational velocity with mass. At $2 \mathrm{Myr}$, the inferred mass for spectral type K1 (from the Siess isochrone; Siess et al. 2000) is $2.5 M_{\odot}$, and $2.1 M_{\odot}$ for $\mathrm{K} 2.5$. These stars will arrive on the main sequence as A stars, with mean rotational velocities of about $150 \mathrm{~km} \mathrm{~s}^{-1}$.

Ignoring the high-mass stars, if one compares only the stars with $g-i>1.3$, there is no obvious difference between the $v \sin i$ distributions for the accretor group and those for the variable extinction group. This seems surprising, since the variable extinction stars are expected to be viewed at high inclination angles and to probably be older on average - both of which should lead to larger spectroscopic rotational velocities. Do accretion models offer an explanation? KR08 discuss the factors that favor burst-dominated light curves, which include high accretion rates, relatively slow rotation, and relatively weak dipole B fields. Favoring slow rotation for the burstdominated sources only exacerbates our problem. For disk- locked CTTSs, Long et al. (2013) predict rotation periods that scale as $M_{\odot}^{3 / 7}$-predicting relatively slow rotation for stars with high accretion rates. Our expectation remains, therefore, that the variable extinction stars should have larger $v \sin i$ value's at a given mass compared to the burst-dominated sources, and we have no explanation for why this is not reflected by the data shown in Figure 12. We note, however, that a similar problem was also present and discussed among the Class III (or UX Ori) variables of H94, whose $v \sin i$ value's also did not appear to be larger on average than non-variable YSOs of the same spectral type.

\subsection{Inferences on System Geometry from Optical/IR Light-curve Correlations}

We have concentrated so far on the CoRoT light curves, because they have the highest cadence and directly detect photons from the accretion-powered hot spots. Our Spitzer light curves also have relatively high cadence and accuracy, and based on model fits to the optical-IR SEDs, for the stars with accretion-burst-dominated light curves of Table 1, the $4.5 \mu \mathrm{m}$ 
Table 4

Predicted Disk Properties at $4.5 \mu \mathrm{m}$ as a Function of View Angle

\begin{tabular}{lcc}
\hline \hline Inclination & Back to Front Flux Ratio & Relative Total $4.5 \mu \mathrm{m}$ Flux \\
\hline 0 & 1.00 & 1.00 \\
30 & 1.52 & 1.27 \\
60 & 2.55 & 1.35 \\
70 & 4.03 & 1.34 \\
\hline
\end{tabular}

flux is expected to come primarily from warm dust in the inner disk.

Our model for the accretion bursts is that they arise from individual, short-lived mass infall events at small spots on the stellar photosphere. Given that model, it follows that the correlation between the optical and IR light curves will depend on the longitude and latitude of the hot spot, our view to the system, the alignment of the stellar and disk rotation angles, and the detailed structure of the inner disk. Empirically, we see considerable variation in the degree of correlation between the CoRoT and Spitzer light curves. This is illustrated in Figure 13 for four stars of Table 1; Figure 22 in the Appendix shows the entirety of the data for the stars of Table 1.

We will not attempt here to enter into a detailed analysis of the Spitzer light curves. Instead, we will only attempt to show that the primary features shown in Figure 13 are compatible with our basic model for the bursts. In order to motivate that discussion, we first describe the expected $4.5 \mu \mathrm{m}$ appearance of a standard flared disk with an inner disk wall as a function of our view angle to that disk, based on disk models by co-I B. Whitney (Whitney et al. 2013). Figure 14 shows images of a typical disk system for a $1 L_{\odot}$ star with $T_{\text {eff }}=4000 \mathrm{~K}$. The radius of the inner disk is set by the dust destruction temperature of $1600 \mathrm{~K}$, and is about 7.9 stellar radii or $0.075 \mathrm{AU}$. The wall height is set by the hydrostatic equilibrium scale height at the same temperature. The disk accretion rate is $8 \times 10^{-8} M_{\odot} \mathrm{yr}^{-1}$. The ratio of disk to photospheric flux at $4.5 \mu \mathrm{m}$ is about 3.0 for these models, nearly independent of inclination angle. Figure 14 shows this disk as seen from three different view angles $-0^{\circ}$, $30^{\circ}$, and $70^{\circ}$. As the view angle increases, both the total surface area of the $4.5 \mu \mathrm{m}$ emitting dust increases and the fraction of that flux emitted by the back wall increases. Table 4 quantifies the dependence of the $4.5 \mu \mathrm{m}$ emission on view angle for this specific disk model. At $70^{\circ}$ view angle, $80 \%$ of the $4.5 \mu \mathrm{m}$ flux we receive from the circumstellar dust comes from the back side of the disk. For inclinations greater than about $75^{\circ}$, the $4.5 \mu \mathrm{m}$ emission from the system drops sharply due to extinction from cold dust in the flared outer disk.

Based on this model, our expectations for the optical and IR light-curve correlations are:

1. For a star with no disk, the CoRoT and Spitzer light curves must be almost perfectly correlated, with the only difference being the wavelength dependence of the different components at or near the star's surface (spots, flares, etc.)

2. For a star with a disk, an important factor is the fraction of the star's flux at 3.6/4.5 $\mu \mathrm{m}$ that comes from warm dust in the disk. Stars with an inner disk hole (no warm dust) should have a different degree of correlation between their CoRoT and Spitzer light curves compared to those with significant warm dust. For this reason, the last column of Table 1 shows our estimate of the ratio of the $4.5 \mu \mathrm{m}$ flux from the disk to that from the star, based on fits to their SEDs for stars where we have spectral types. Not surprisingly given their other characteristics, with only one exception (Mon 860, - for
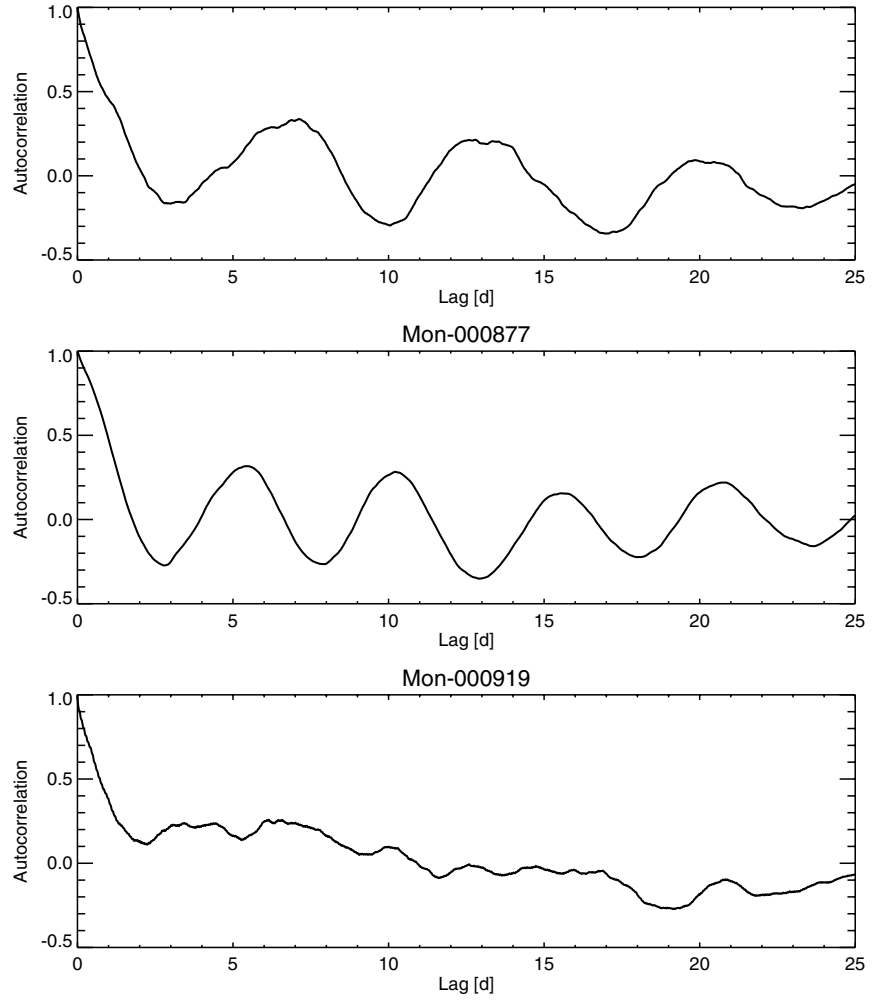

Figure 19. Auto-correlation functions (ACFs) for two of the accretion burst stars, Mon-000406 (top) and Mon-000877 (middle). This illustrates that the bursts for these stars are preferentially clumped together with a periodic spacing. The ACFs for most of the burst-dominated light curves do not show any significant evidence of periodic structures, as illustrated by the ACF for Mon-000919 (bottom).

which we have no synoptic IRAC data), the disk dominates the flux at $4.5 \mu \mathrm{m}$. However, many of the other stars have disk/star flux ratios of only 2-3, so even though the disk dominates, large features in the CoRoT light curves should still have something present in the Spitzer light curve.

3. At most view angles to the disk, it is quite possible to have a transient hot spot on the back side of the star illuminate the back-side inner disk, thereby yielding a flux burst in the IRAC light curve, but because the hot spot is outside our direct view, there is no imprint on the CoRoT light curve (e.g., the features in the Mon 117 light curve near day 25 and day 30).

4. For disks seen at large inclination angles, it is possible to have a transient hot spot on the side of the star facing us cause a CoRoT flux burst, but because the $4.5 \mu \mathrm{m}$ emission is heavily dominated by the back-side of the disk where we have a better view to the inner disk wall, there would be little or no imprint of the burst on the IRAC light curve (e.g., the feature in the Mon 1022 light curve near day 24).

\section{ACCRETION PROPERTIES DERIVED FROM THE LIGHT CURVES}

The CoRoT light curves contain a considerable amount of quantitative information related to the accretion events. We discuss a number of physical constraints on the accretion process derived from the light curves in the next several sections. 

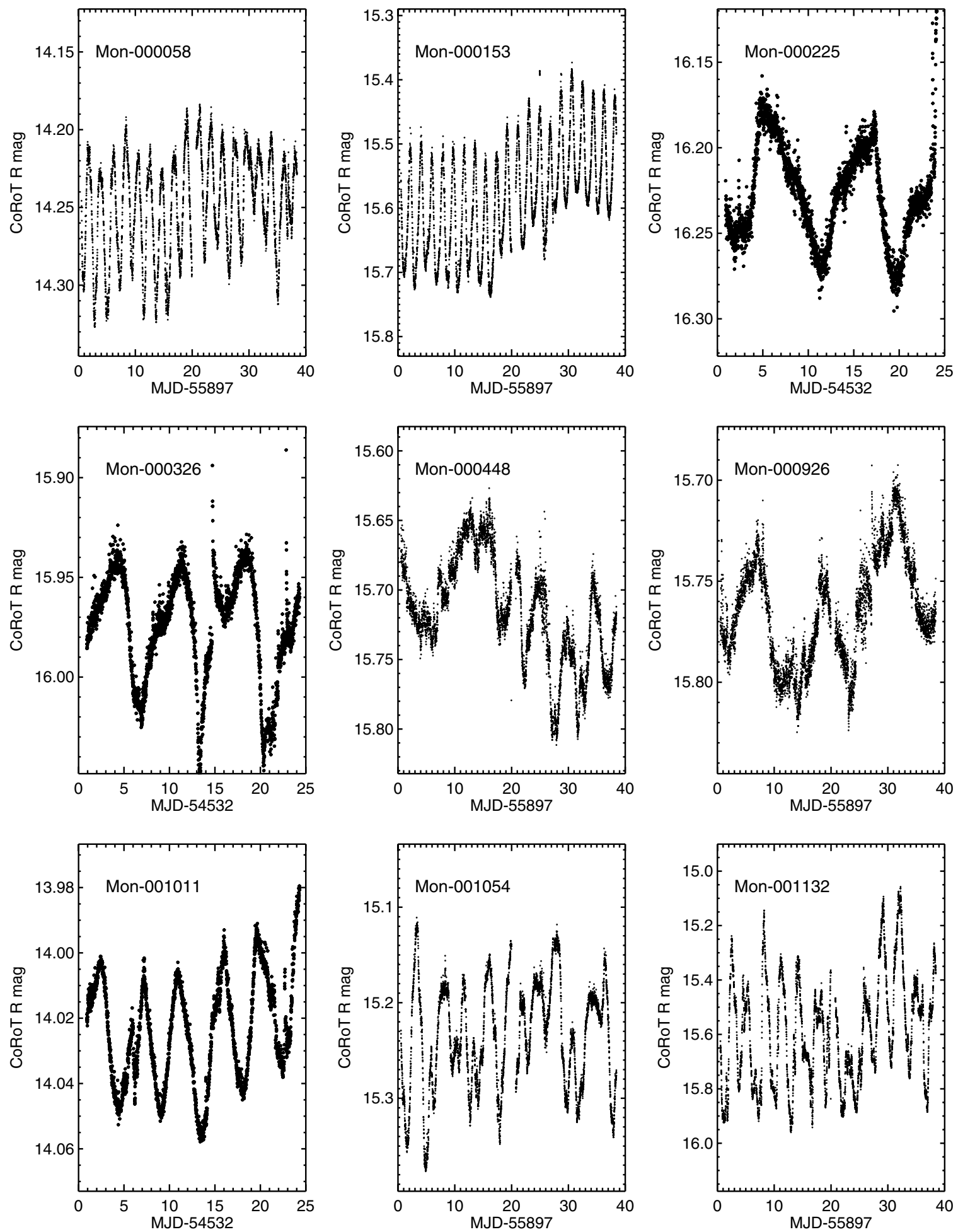

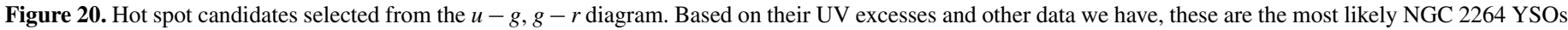
to have relatively stable hot spots due to funnel-flow accretion.

\subsection{Burst Durations}

The duration and amplitude of the individual flux bursts in the light curves for the stars from Table 1 encode information related to the mass and size scale of the individual gas streams accreting onto the photosphere. While it is often the case that the light-curve structures are too complicated to identify individual events, in some cases what appear to be individual bursts are present. We have carefully examined all of the CoRoT light curves of the stars in Table 1, and we report those results here.

The CoRoT high-cadence mode (sample rate of once per $32 \mathrm{~s}$, or 16 times faster than the normal cadence) was utilized for only one star in Table 1; that star was Mon-000474. Mon-000474 is 

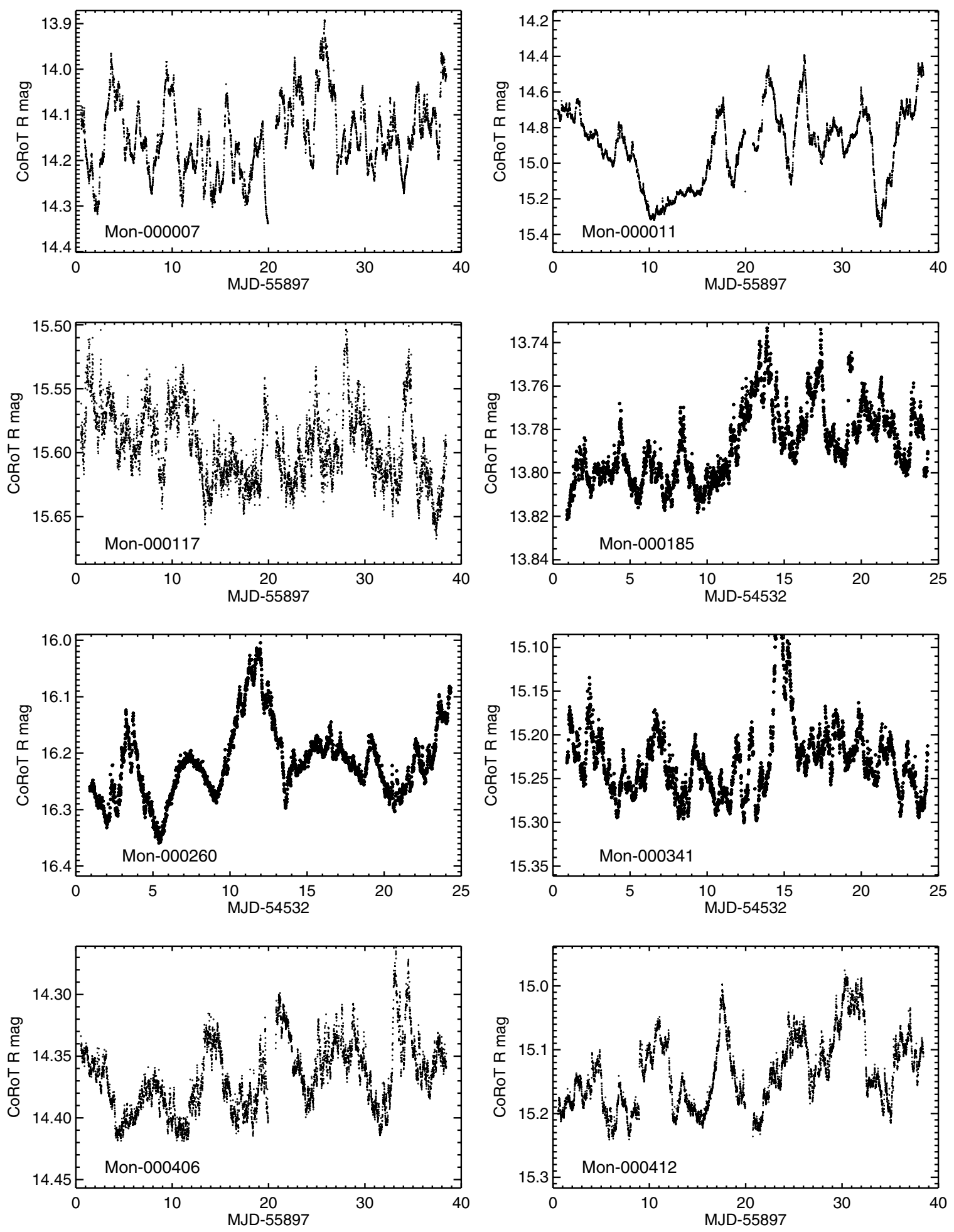

Figure 21. CoRoT light curves for all the stars of Table 1. We have converted the CoRoT "counts" into an estimated $R$ magnitude using a zero point of 26.74 .

also (not coincidentally) by far the brightest of the Table 1 stars, with an $R$ magnitude more than 3 magnitudes brighter than the average for the other stars of Table $1 .{ }^{23}$ This combination makes the Mon-000474 light curve much more sensitive to

${ }^{23}$ Mon-000474 is also the earliest spectral type star in the accretion burst group. It is possible that burst duration is a function of spectral type, in which case the inferences we draw from Mon-000474 may not be applicable to our entire sample. With only one star observed in high-cadence mode, we ignore this possibility for now. shorter duration, lower luminosity light-curve structures. The complete CoRoT light curve for Mon-000474 is shown in the Appendix in Figure 21. Figure 15 (top row) shows two very small segments extracted from that light curve, with nine shortduration flux bursts that we ascribe to accretion variability marked with red bars. Also present in this time window in the CoRoT light curve is another event of a different character, shown in Figure 15(c). This event is a well-observed stellar flare, with a very fast rise time (the "impulsive" phase), followed 

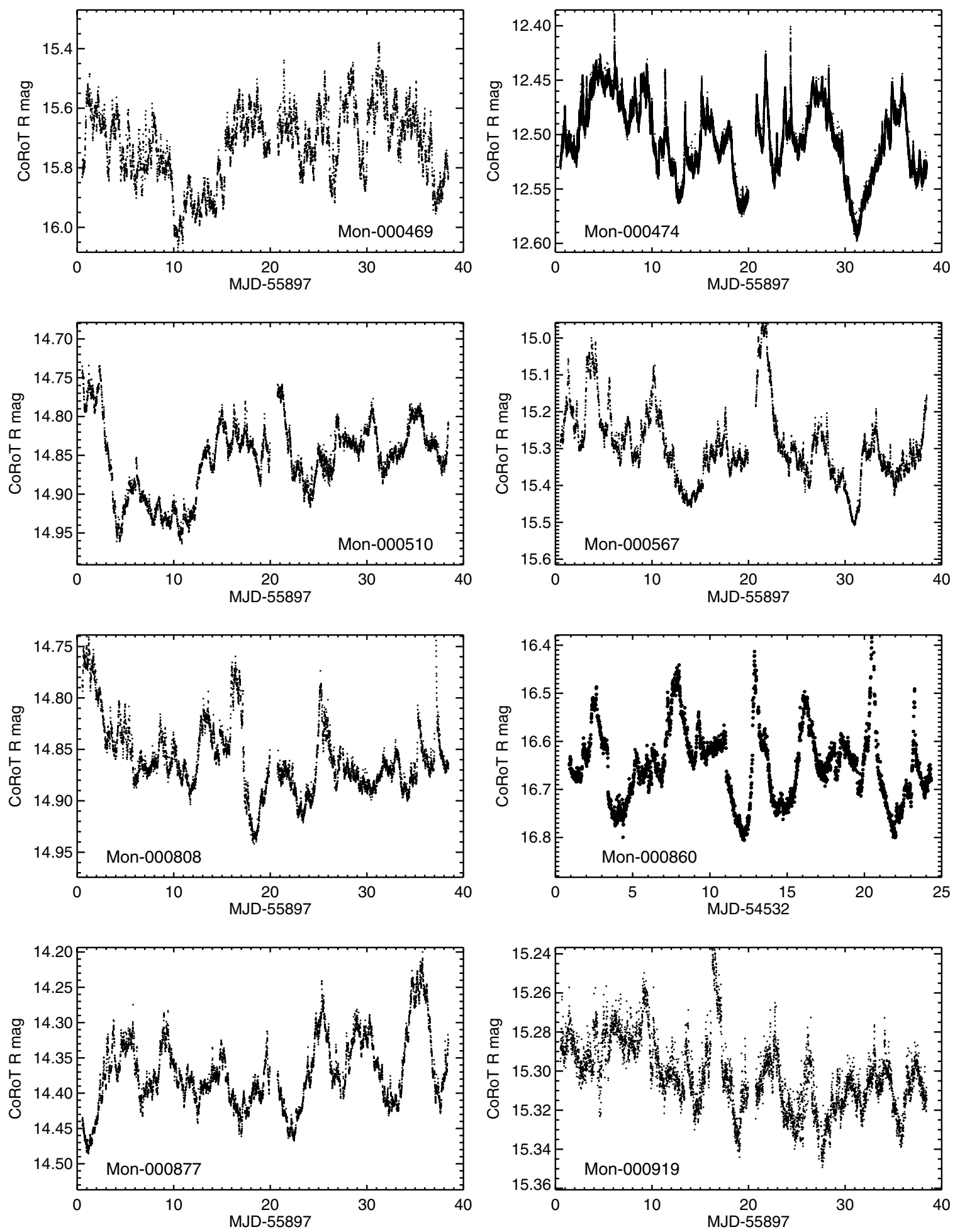

Figure 21. (Continued)

by an initially fast fall and then a much slower decay (the "gradual" phase). At CoRoT"s high cadence, stellar flares are easily distinguished from even short-duration accretion bursts. At the more normal low cadence (512 s sampling), our ability to identify short-duration bursts in the Mon-000474 light curve and to discriminate them from flares would be much reduced. Figure 15(d) provides a direct comparison by showing a portion of the Mon-000474 light curve, but only plotting every 16th point, thereby better matching both the cadence and signal-to- noise ratio of the other burst-dominated light curves. The red dots mark the accretion bursts we had identified in Figures 15(a) and (b); only one of those bursts (the last one) is clearly identifiable at this cadence. The blue dots mark features we would identify as isolated accretion bursts at this cadence. Unfortunately, one of them (the point at day 6.1) is the stellar flare illustrated in Figure 15(c). At the lower, normal cadence, a stellar flare can be mistaken for a short-duration accretion burst. 

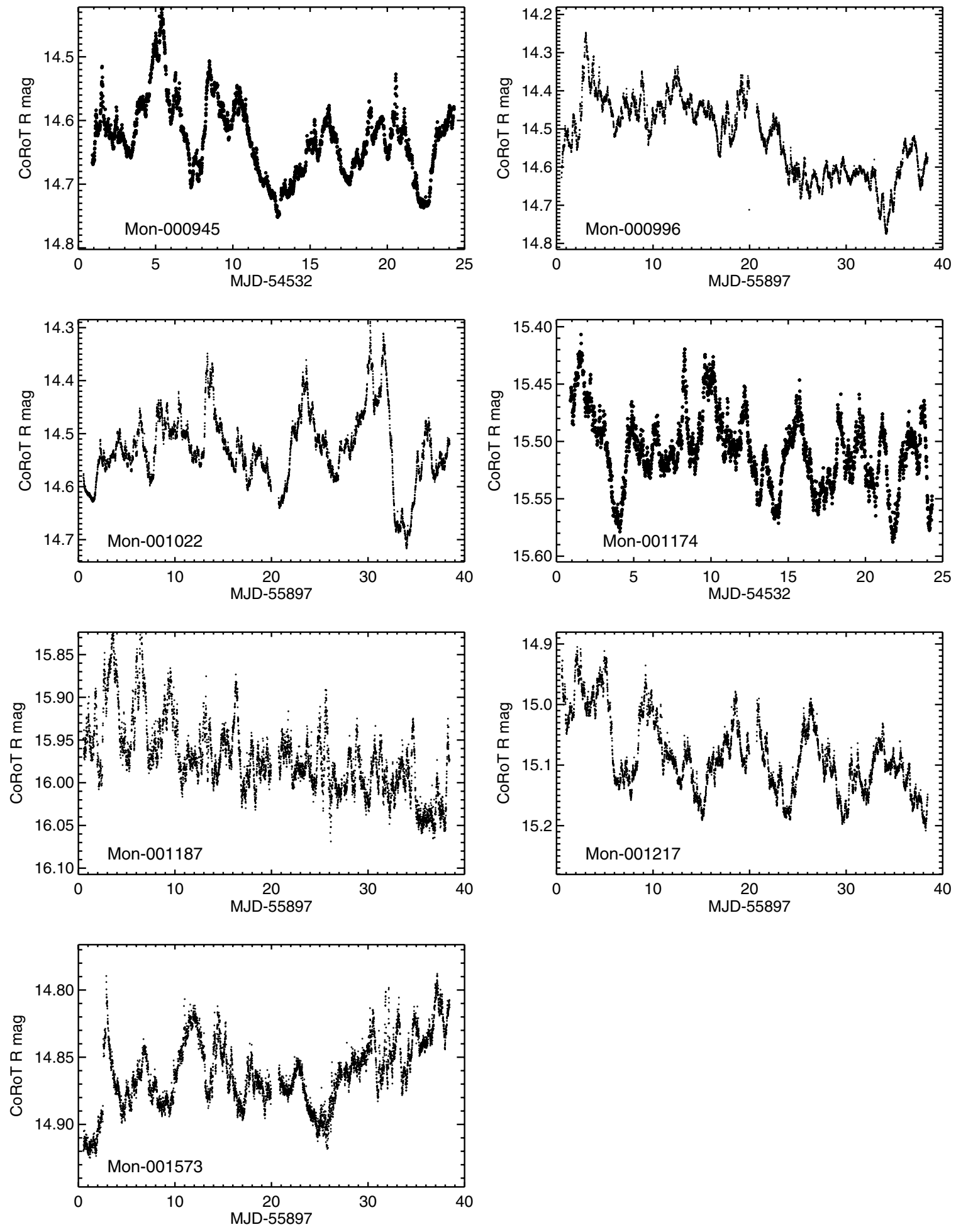

Figure 21. (Continued)

With the experience gathered from the Mon-000474 light curve, we have made a careful examination of the lower cadence CoRoT light curves for all of the other stars from Table 1. In order to avoid possible contamination from flares, we have not included any feature with width less than 0.2 days. Figure 16 shows nine of the events classified as accretion bursts in the lowcadence data.

Figure 17 shows histograms of the rise times (time from our estimate of when the burst begins to the mid-point) and fall times (time from the mid-point to our estimate when the burst completes) for the events measured for Mon-000474 (Figure 17(a)) and for the remaining 23 accretion burst stars (Figure 17(b)). For Mon-000474, the median rise and fall times are both 0.08 days; for the other stars, those medians are 0.4 and 0.5 days. The amplitudes of the peaks identifiable in Mon000474 and the other stars are also systematically different. Using the ratio of the height of the peak relative to the local continuum as the measure, the mean for the Mon-000474 bursts 
Table 5

Light-curve Types for Stars with Definite UV Excess

\begin{tabular}{lrc}
\hline \hline Light-curve Type & N & Description \\
\hline Constant & 2 & Essentially constant \\
Low amplitude variable & 5 & definitely variable, but noisy and ampl. small \\
Stochastic & 9 & highly variable (see Cody et al. 2014) \\
Burst-dominated & 24 & 22 stars from Table 1 + 2 additional low-ampl bursts \\
Extinction dip stars & 10 & see Figure 20 \\
Hot Spot Candidates & 9 & \\
\hline
\end{tabular}

Notes. Stochastic = adopted from Cody et al. (2014), stars whose light curves show often large-amplitude variability but without preference for positive or negative flux changes. These stars probably have several mechanisms contributing to the observed variability.

Table 6

Basic Information for YSOs Whose Light Curves May be Dominated by Stable, High-latitude Hot Spots

\begin{tabular}{|c|c|c|c|c|c|c|c|}
\hline Mon ID ${ }^{\mathrm{a}}$ & R.A. & Decl. & 2MASS ID & CoRoT 2008 & CoRoT 2011 & $\mathrm{SpT}^{\mathrm{a}}$ & $\begin{array}{c}\mathrm{H} \alpha \mathrm{EW}^{\mathrm{a}} \\
(\AA)\end{array}$ \\
\hline Mon-000058 & 100.53625 & 9.689221 & $06420870+0941212$ & null & 616895632 & $\ldots$ & 94.0 \\
\hline Mon-000153 & 100.24962 & 9.784611 & $06405990+0947044$ & null & 400007889 & M3 & 39.9 \\
\hline Mon-000225 & 100.27596 & 9.417666 & $06410622+0925036$ & 500007896 & null & M5 & 34.7 \\
\hline Mon-000326 & 100.24508 & 9.655193 & $06405882+0939187$ & 223980258 & 223980258 & M0 & 27.9 \\
\hline Mon-000448 & 100.26500 & 9.508055 & $06410360+0930290$ & 400007803 & 602083897 & $\ldots$ & 20.4 \\
\hline Mon-000926 & 100.27679 & 9.477443 & $06410642+0928388$ & 400007686 & 400007687 & M1.5 & 56.1 \\
\hline Mon-001011 & 100.16887 & 9.583666 & $06404052+0935011$ & 500008145 & null & $\ldots$ & $\ldots$ \\
\hline Mon-001054 & 100.15221 & 9.845999 & $06403652+0950456$ & 400007538 & 400007538 & M2 & 21.1 \\
\hline Mon-001132 & 100.10779 & 9.849332 & $06402587+0950576$ & null & 602095740 & $\mathrm{M} 2.5$ & 166.0 \\
\hline
\end{tabular}

Note. ${ }^{a}$ See Cody et al. (2014) for the sources of the spectral type and H $\alpha$ equivalent width (EW) data. All of these are in emission.

is $2.3 \%$, whereas for the other 23 stars observed at low cadence, the mean is $10.6 \%$. The burst durations and amplitudes measured in the standard cadence data match well to the models of KR08 and R12; the much shorter duration and lower amplitude bursts visible in the high-cadence, high signal-to-noise ratio Mon000474 data were not expected based on those models.

\subsection{Contribution to the Stellar Flux from Accretion Bursts}

What fraction of the broad-band optical light output of the stars from Table 1 can be ascribed to the short-duration accretion bursts that are visible in the CoRoT light curves? How does the integrated flux from the discrete bursts that are identifiable in the light curves compare to the veiling flux inferred from the VLT spectra? We can make an estimate of this fraction by adopting a pseudo-continuum level as a function of time for the CoRoT light curves, and then integrating the "flux" above that level. Setting that continuum level is somewhat arbitrary, particularly for stars where there is considerable structure not necessarily due to accretion bursts. For our purposes, however, we argue that it is sufficient to do this estimate in a perhaps "generous" and simplified manner. Specifically, we adopt a constant flux level for a portion (or all) of the light curve where that constant level seems at least plausible as the continuum above which the identifiable bursts are superposed. We have made these estimates for all six stars of Table 1 for which we have both CoRoT light curves in 2011 and VLT/FLAMES spectroscopy. We illustrate our method in Figure 18. The solid line is our estimated pseudocontinuum level. For these six stars, the estimated fraction of the broad-band optical light from accretion bursts ranges from $4.9 \%$ to $14 \%$. In other words, the individually identifiable accretion bursts are responsible for only a small fraction of the optical broad-band light, at least by this metric.
As we have noted previously, the characteristic veiling factor for these stars is $r=0.65$, corresponding to a prediction that $39 \%$ of the optical flux derives from hot gas. The red dashed lines in Figure 18 correspond to the continuum level that would be needed to match this estimate for the veiling continuum contribution to the CoRoT light curves. We conclude that either there are a large number of small accretion bursts that blend together to contribute to the veiling continuum, or there is some other, more continuous accretion mode that is contributing the majority of the veiling continuum. The large number of small bursts present in the Mon-000474 light curve suggests that the former explanation may be correct.

\subsection{Timescales and Periodicities for the Accretion Bursts}

Autocorrelation function (ACF) analysis for time series data provides a sensitive means to search for periodic signals. We have derived ACFs for all of the stars in Table 1. Prior to doing that, as is standard, we have resampled the data to a regular cadence and we have subtracted the median (see Cody et al. 2014).

Most of the burst-dominated light curves do not show a significant periodic signal, i.e., the bursts occur stochastically. However, four of the stars do have strong periodicities: Mon000406 ( $P=6.2$ days $), 412(P=6.6$ days $), 877(P=$ 4.6 days), and 1217 ( $P=7.0$ days). The ACFs for two of these stars are shown in Figure 19. Because of their periodicity, one might associate these light curves with funnel-flow accretion channeled by the stellar dipole magnetic field. However, these light curves are quite unlike what is commonly envisioned for stable funnel-flow accretion (see Figure 7(a) in KR08), where the variations are more nearly sinusoidal, but they could represent an intermediate state where there is a dominant matter flow controlled by the magnetic dipole, but disk instabilities 

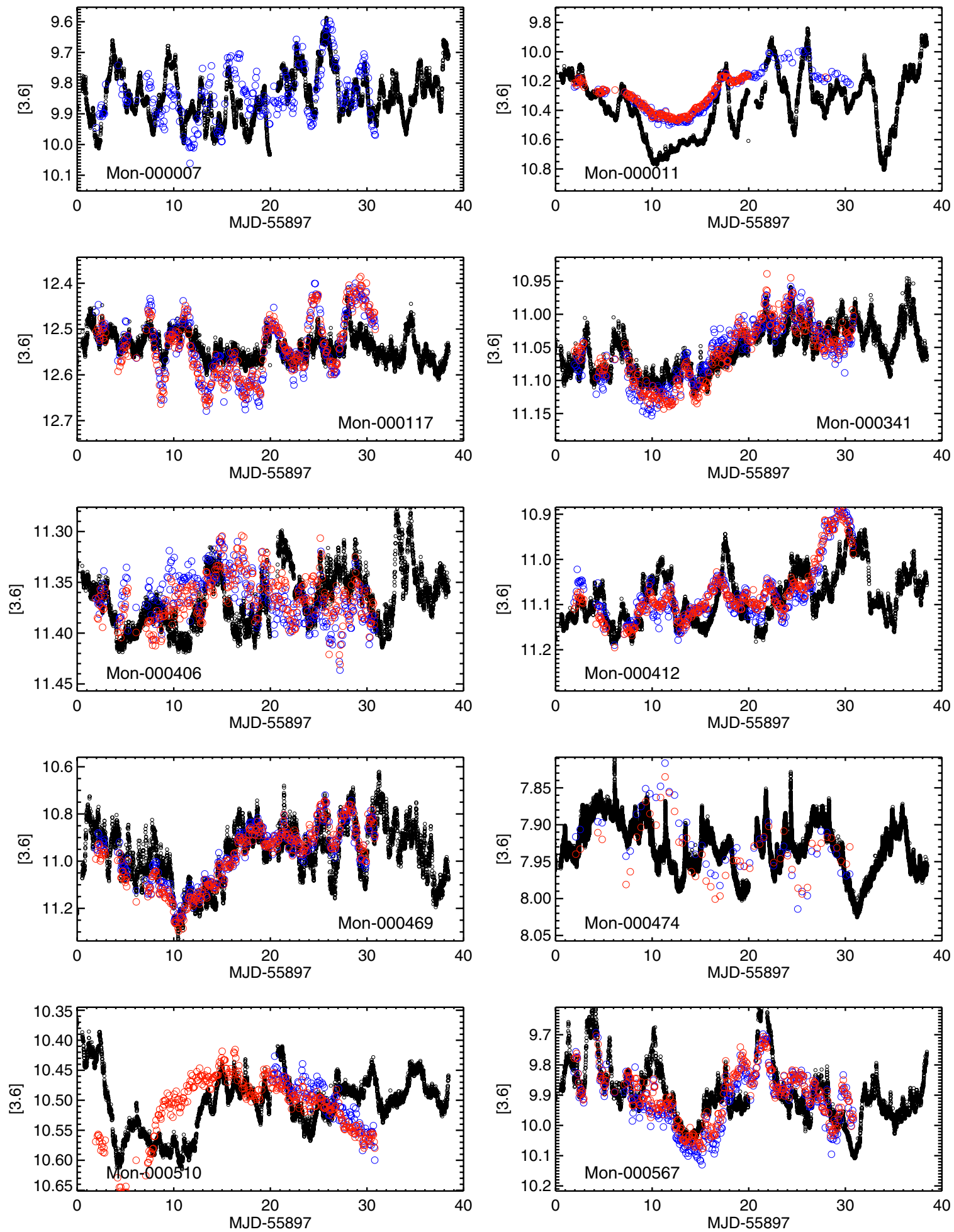

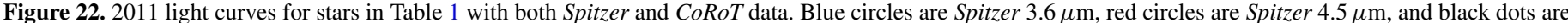
CoRoT data. See discussion in Section 5.4. In most cases, there is a fair correlation between the shape and amplitudes of the light curves at the two wavelengths.

(A color version of this figure is available in the online journal.)

lead to an unsteady flow of gas toward the stellar surface (quasi-periodic variability of this type was also predicted by KR08; D'Angelo \& Spruit 2010 propose another accretion disk instability that can produce periodic accretion bursts). Whatever is driving the periodicity in these stars is apparently long-lived, because three of the four (Mon-000406, 877, and 1217) have very similar periods published in the literature (L04; Makidon et al. 2004). We assume that the derived periods correspond to the photospheric rotation period for these stars.

An interesting, but difficult to quantify, statistic to determine for the burst-dominated light curves is the average frequency for the bursts. Based on the analysis of the high signal-tonoise ratio, high-cadence light curve for Mon 474, the number of bursts one can identify is a strong function of the 

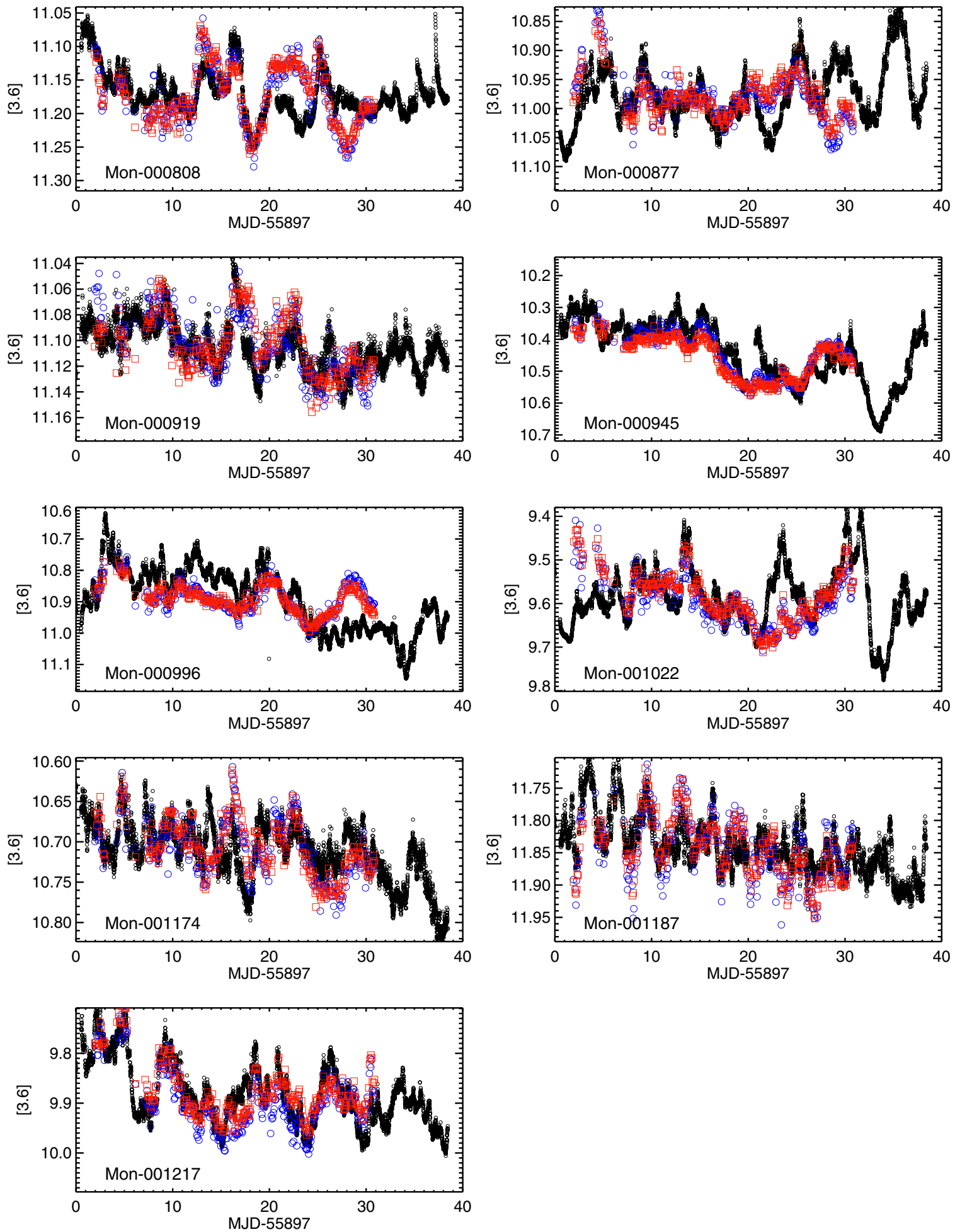

Figure 22. (Continued)

light-curve quality. Therefore, it is important to utilize a quantitative algorithm to provide a consistent, though somewhat arbitrary, burst frequency. For this purpose, we use the PeakFind routine defined and described in Cody et al. (2014). This algorithm counts the number of peaks in a light curve that differ in magnitude by more than a particular amplitude. The number of peaks is then divided by the total time baseline of the light curve to produce a timescale. Repeating for a grid of amplitudes results in a timescale distribution for peaks of different mini- mum size. For the burst-dominated light curves, PeakFind does a reasonably good job of identifying the same relatively strong, relatively isolated peaks that were measured to create Figure 17. For the three burst-dominated light curves shown in Figure 1, PeakFind identified 5 (Mon-000567), 8 (Mon-000808), and 10 (Mon-001187) bursts. For the entire set of stars in Table 1, the median burst frequency determined by PeakFind was 0.2 peaks per day, corresponding to $\sim 8$ peaks in the CoRoT observing window. This is roughly comparable to the burst fre- 

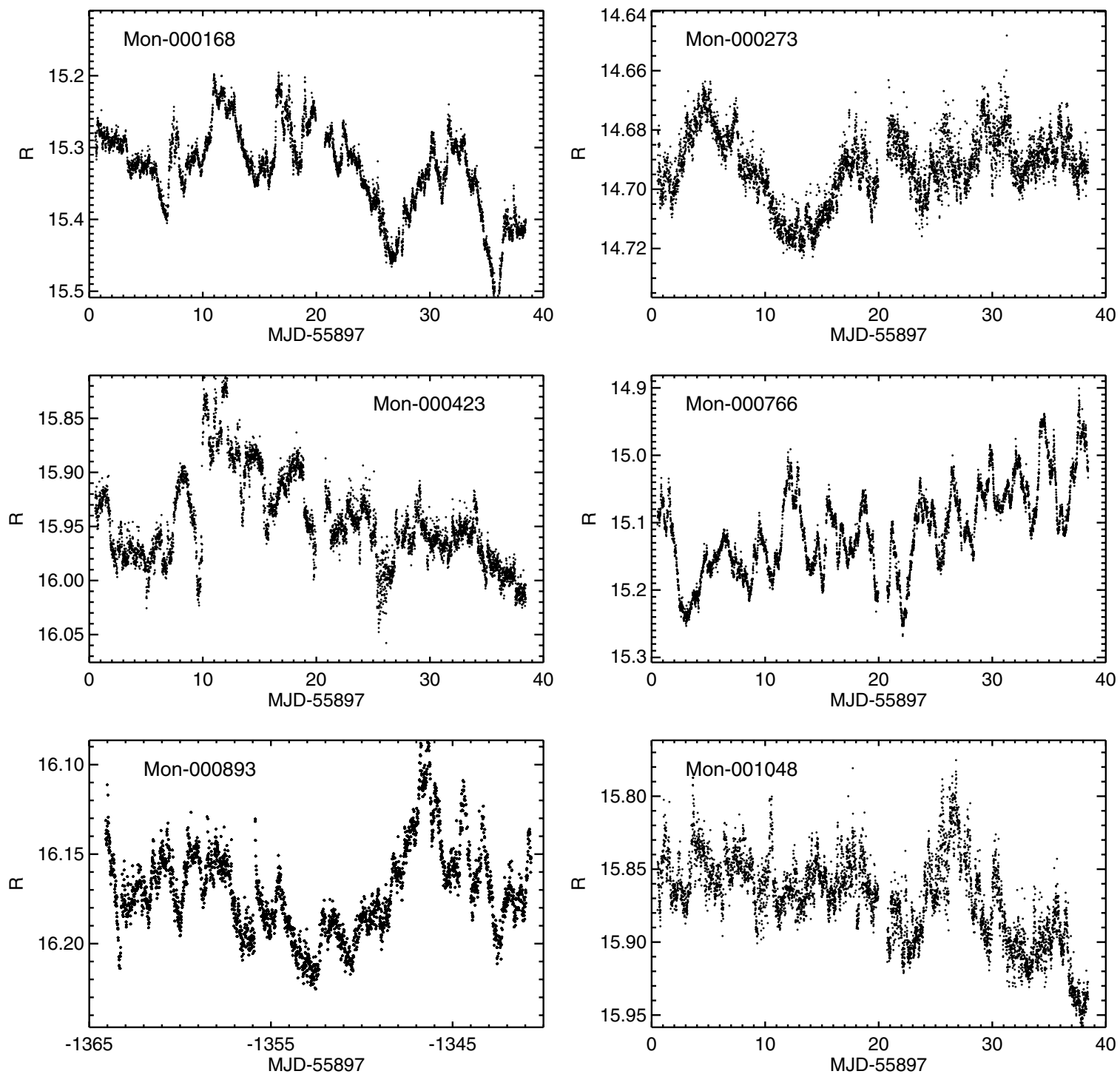

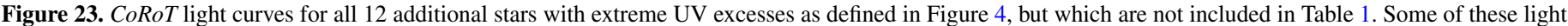

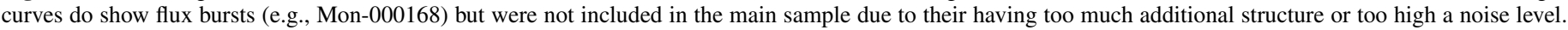
See the discussion in Section 4.

quency displayed in the model stochastic light curves of R12 and KR08.

\section{POPULATION STATISTICS—THE FREQUENCY OF CTTS LIGHT-CURVE TYPES}

In Section 4, we noted that among the stars with the largest UV excesses, the stars with accretion burst light curves were by far the dominant light-curve class (at least 15 out of 27 stars), and that only one of the extreme UV excess stars could be interpreted as being dominated by a stable high-latitude hot spot as is expected from funnel-flow accretion. Here we conduct a more complete comparison of the frequency of light-curve types as it relates to the accretion process.

To create a larger sample of CTTSs from which to determine the relative frequency of various light-curve types, we have drawn a second boundary in the $u-g, g-r$ plane, meant to separate stars with certain UV excesses from those that have either no UV excess or only a slight UV excess. This line, approximately parallel with the locus of WTTSs in the $u-g$, $g-r$ plane, is shown as a dotted line in Figure 4(d). There are
60 stars that we believe are NGC 2264 members and for which we have CoRoT light curves whose $u g r$ colors place them below the line. We have made a visual characterization of all 60 light curves, which we summarize in Table 5. Only nine of these stars have light curves whose shapes are possibly consistent with that expected for a relatively stable high-latitude hot spot; these light curves are shown in Figure 20. To fall into this category, we only require that the light curve shows apparently periodic structure, and that it is not better categorized as an AA Tau-analog or an accretion burst star.

Can we be sure that the light-curve shapes in Figure 20 are, in fact, the result of hot (and not cold) high-latitude spots? For WTTSs, cold spots are generally stable over many rotation periods. Herbst et al. (1994) and others have reported that spotted light curves on CTTSs are much less stable, possibly because they are the result of hot spots that result from intrinsically variable accretion. There is, therefore, a prejudice to suspect that the more consistent the light-curve shape from one period to the next, the more likely that cold spots are responsible. However, cold spots may behave differently on CTTSs, so this is not a certain conclusion. Independent of light-curve shape, 

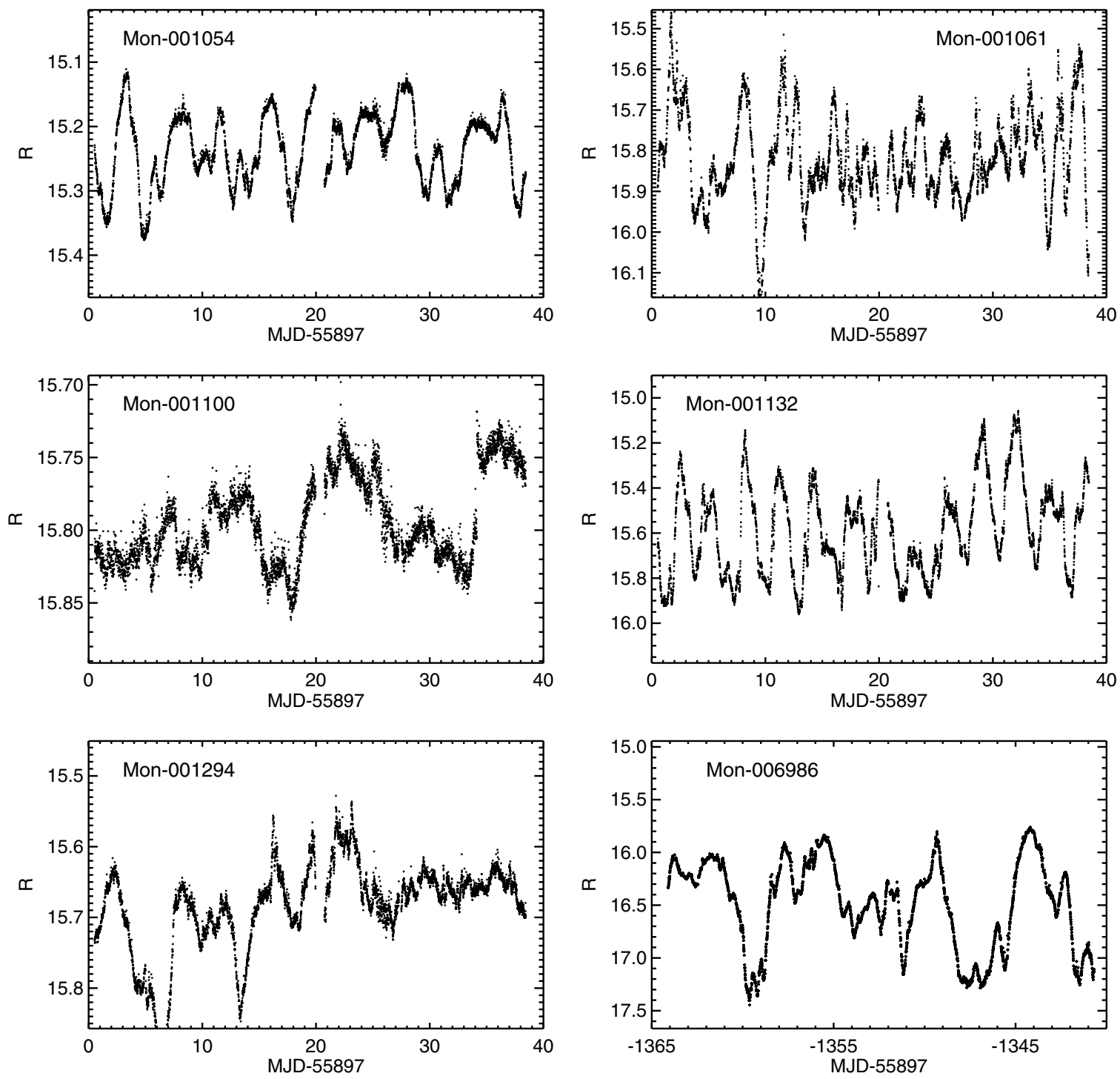

Figure 23. (Continued)

well-sampled synoptic $U$-band photometry or high-resolution spectroscopy can discriminate between cold and hot spots, but our access to such data for these stars is limited. What we can do is simply ask that these hot spot candidates have properties that are consistent with having accretion contribute significantly to their light-curve shape, using the accretion burst stars as a guide.

First, we require that the star have evidence of warm dust (as do all of the stars in Table 1) - that is, it does not lie in the Class III locus in the IRAC color-color diagram, or if not all four IRAC bands have data, then there is evidence in the SED for an IR excess beginning somewhere shortward of $\sim 10 \mu \mathrm{m}$. If we have VLT spectra (true for five of these stars), then the star must be detectably veiled $-r>0.2$; we also expect these stars to show He I $\lambda 6678$ in emission (as do all of the stars from Table 1 with VLT spectra). If we have $\mathrm{H} \alpha$ profiles, then the profile must be detectably structured or broad. All nine of these candidates pass these tests, where we have the appropriate data. Table 6 provides the coordinates and other information for these stars. These nine stars are the best candidate funnel-flow accretors among the 60 stars with certain UV excesses, though it is possible that for some stars the light-curve shape could be due to evolving cold spots or other mechanisms. We conclude that for the NGC 2264 members with certain UV excesses, lightcurve morphologies suggestive of funnel-flow accretion occur only about one-third as often as light curves dominated by shortduration accretion bursts. Depending on what fraction of these stable hot spot candidates are confirmed, funnel-flow accretion may dominate at relatively low accretion rates, matching the prediction of KR08.

Cody et al. (2014) used an ACF technique to identify NGC 2264 CTTSs whose CoRoT light curves are periodic or quasi-periodic. The latter are light curves where there is a periodic signal, but either the shape or amplitude of the waveform changes significantly over the 40 day CoRoT shortrun (see Cody et al. 2014 for the quantitative details). Only five CTTSs were identified as having periodic CoRoT light curves. All five of these stars fall within the WTTS locus in the $u-g$ versus $g-i$ color-color diagram, indicating very low (or no) accretion and making it likely that their light-curve shapes are probably due to cold spots. All but two or three of the 27 quasi-periodic CTTS's identified by Cody et al. (2014) 

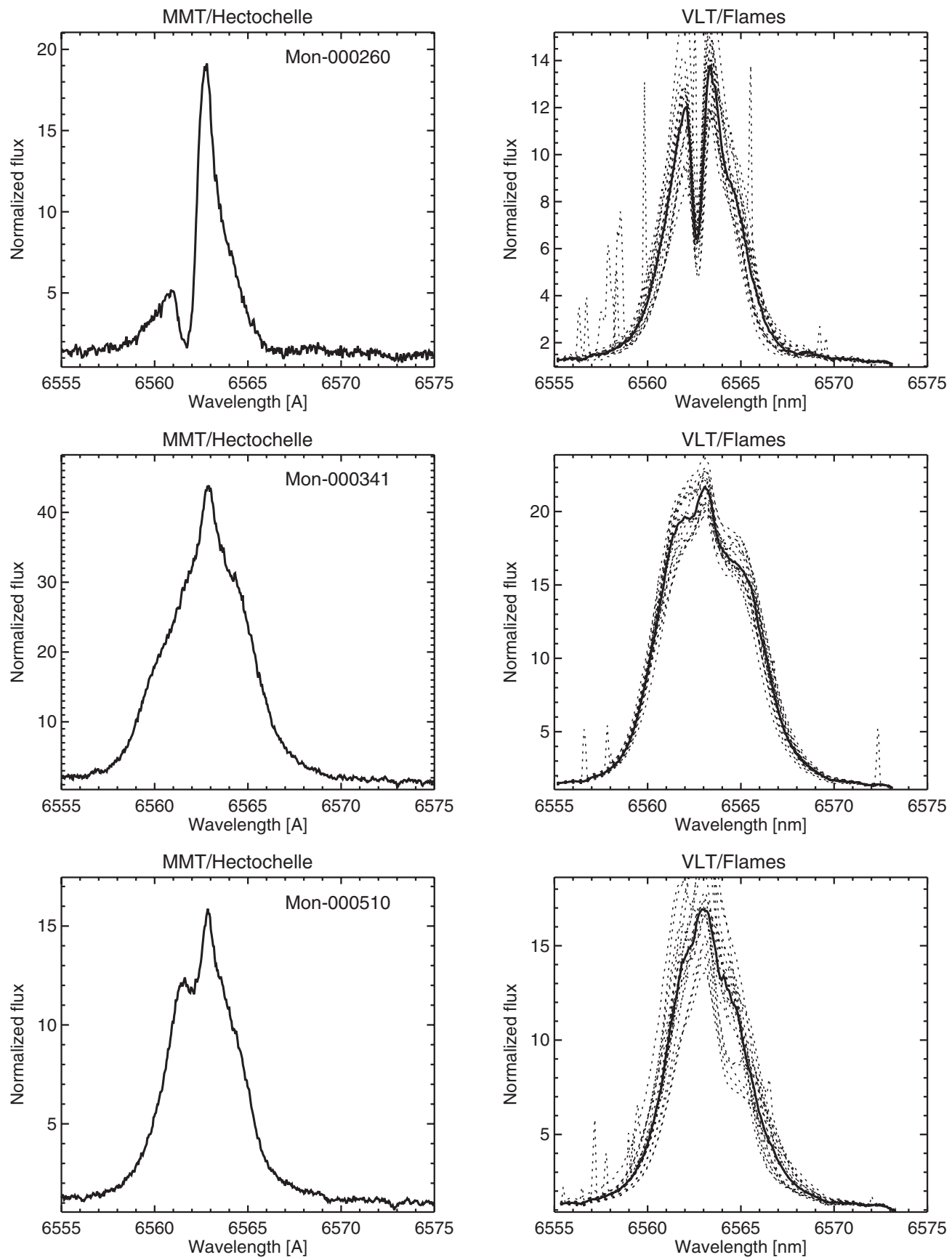

Figure 24. Comparison of $\mathrm{H} \alpha$ profiles for objects with both MMT/Hectochelle data from 2004 or 2005 and VLT/Flames data from 2011/2012.

fall in the $u-g$ versus $g-i$ diagram above the dashed red line of Figure 4(d), but in most cases below the WTTS locus. Therefore, they are probably accreting but at a low rate; their light-curve shape could be due to either to cold or hot spots. In any event, they reinforce the conclusion that stable, funnel-flow accretion — when present - is likely only to occur for stars with low accretion rates.

\section{CONCLUSIONS AND SPECULATION}

Previous synoptic photometric surveys of star-forming regions have provided many useful results constraining the physical properties of YSOs. However, ours is the first such survey to have the necessary cadence, sensitivity, duration, and wide-field and multi-band nature to unmask the dominant modes by which YSOs accrete gas from their circumstellar disk. Our study has also benefited from having high-quality, multi-wavelength photometry from $u$ to $24 \mu \mathrm{m}$ for most of the NGC 2264 members, and high-resolution spectroscopy at least at $\mathrm{H} \alpha$, also for a large fraction of the stars of interest.

Using the $u-g, g-r$ two-color diagram to identify the most actively accreting YSOs in NGC 2264, we have shown that among the YSOs in NGC 2264 with the largest UV excesses, the dominant light-curve signature is short- duration accretion bursts as exemplified by the stars of Table 1. Usually these accretion bursts occur in a seemingly stochastic pattern at least over 40 day timescales; however, for a small fraction of the stars, the accretion bursts are clumped together, with the clumps 

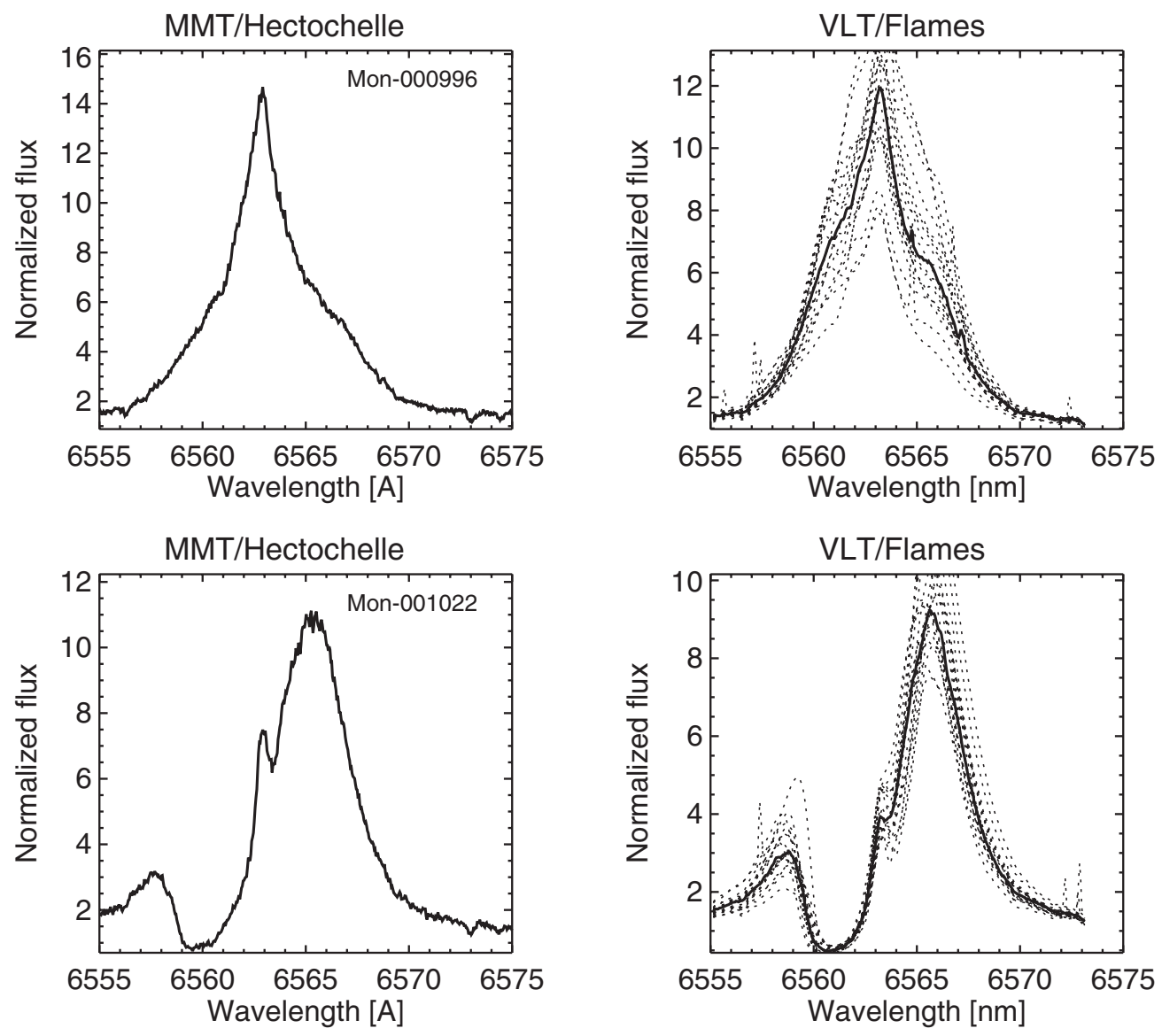

Figure 24. (Continued)

recurring at a period that is plausibly the rotation period of the star. Based on theoretical models, at lower accretion rates we expected to find other accreting stars with long-lived highlatitude hot spots (from funnel-flow accretion) whose light curves would more closely resemble the spotted light curves of WTTSs. We do find a set of candidate stable hot-spot light curves that may correspond to this prediction, but we do not have sufficient data to confirm that hot rather than cold spots are responsible for their variability.

The two most frequent light-curve types among the CTTSs with significant UV excesses are the accretion burst group and the variable extinction group. The latter consists of stars with periodic flux dips (AA Tau analogs) and stars with similar flux dips but no obvious periodicity; in both cases, the flux dips are likely associated with transient structures in their inner circumstellar disk. By their nature, the variable extinction stars must have their disks oriented at relatively large inclination angles to our line of sight. However, we have also discovered several other physical properties that distinguish the two groups. Based on their spatial locations, the variable extinction stars appear to be, on average, older than the accretion burst stars. The variable extinction stars also have smaller UV and IR excesses, perhaps reflective of their greater age. The $\mathrm{H} \alpha$ profiles of the accretion burst group are, for the most part, centrally peaked and modestly structured, with the most common additional feature being blueshifted absorption troughs. The $\mathrm{H} \alpha$ profiles of the variable extinction group are significantly more structured, with about a quarter showing redshifted absorption troughs. When we have the appropriate data, the accretion burst stars all have He I $\lambda 6678$ in emission, whereas the stars with variable extinction generally show an absorption feature, presumably due to Fe I, at that wavelength.

Using the normal cadence CoRoT data, the typical total durations for isolated accretion bursts among the stars of Table 1 are about one day, as predicted by the models of R12. However, for the one star from Table 1 where we have much higher cadence CoRoT data, we identify many shorter duration, low-amplitude bursts that we believe are also best explained as accretion bursts, with total durations as small as a few hours. For at least this one star, which has a much earlier spectral type than the other stars with burst-dominated light curves, the burst distribution function is dominated by these small bursts. For the six stars from Table 1 for which we have VLT spectra and therefore can estimate veiling, we find that only a small fraction of the $R$ band excess light that arises from hot gas can be attributed to the directly identifiable bursts. This could indicate that most of the accretion flux arises from a large number of short-duration, lowamplitude bursts (a hypothesis reminiscent of the debate over whether nano- and microflares power the coronae of low-mass stars; - Hudson 1991; Tajfirouze \& Safari 2012).

This work is based on observations made with the Spitzer Space Telescope, which is operated by the Jet Propulsion Laboratory, California Institute of Technology, under a contract with NASA. Support for this work was provided by NASA through an award issued by JPL/Caltech. 

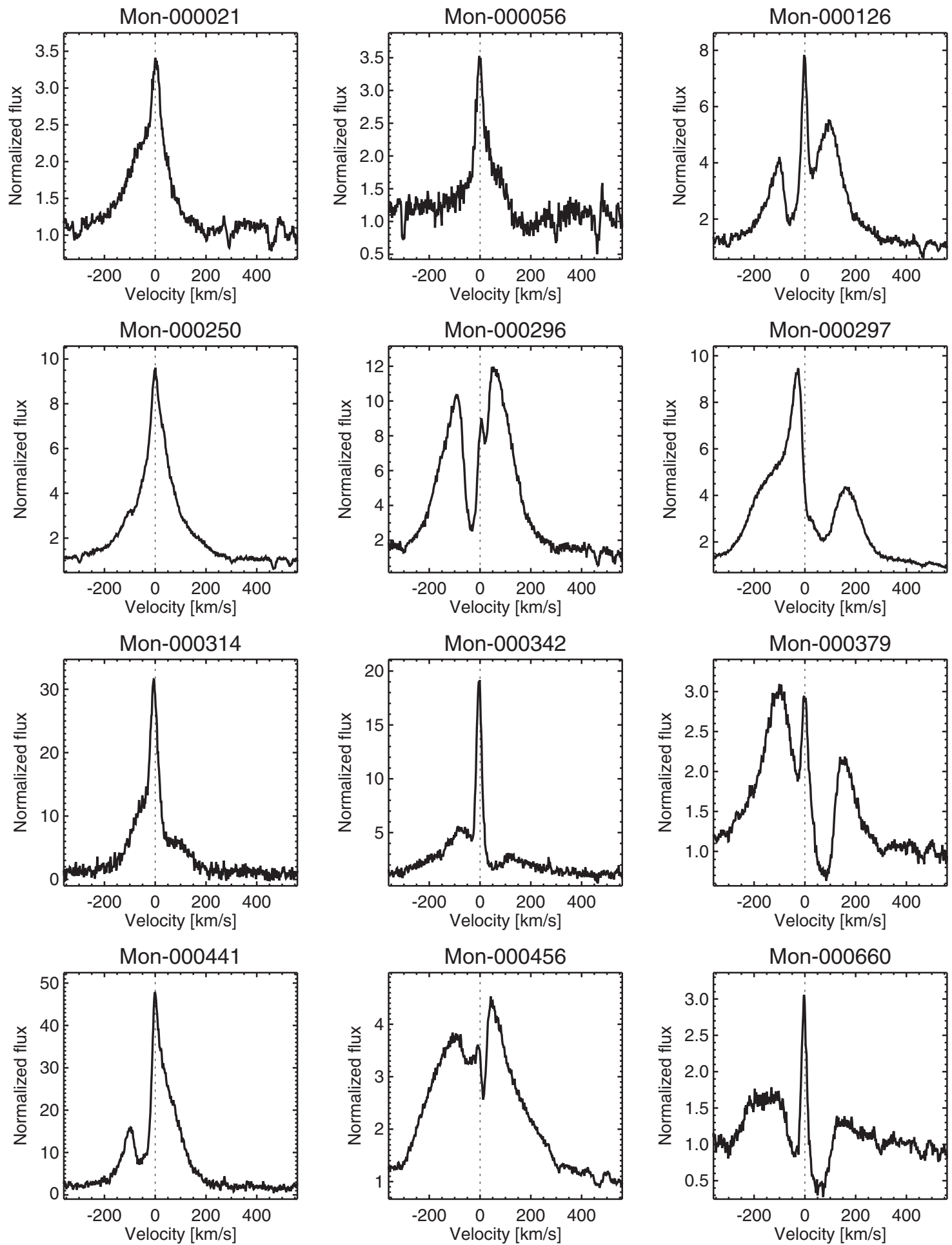

Figure 25. $\mathrm{H} \alpha$ profiles of stars whose CoRoT light curves indicate the presence of prominent extinction dips. The narrow H $\alpha$ emission peaks at rest velocity in Mon $126,314,342,372,660,811$, and 824 may be spurious because of the lack of sky subtraction in the MMT spectra.

\section{APPENDIX}

\section{A.1. CoRoT and Spitzer Light Curves for CTTSs in NGC 2264}

While we display light curves for small subsets of NGC 2264 CTTSs in the main body of the text, we believe it is important to provide light curves for the full set of stars we have discussed. The next three figures provide the full light curves for all of the stars identified as having burst-dominated light curves, plus light curves for the stars with the most extreme UV excesses whose light curves were not identified as being burst-dominated (see Section 4).

Figure 21 provides the CoRoT light curves for all stars from Table 1. Where we have data from both epochs, we show the light curve that best illustrates the accretion burst class. 

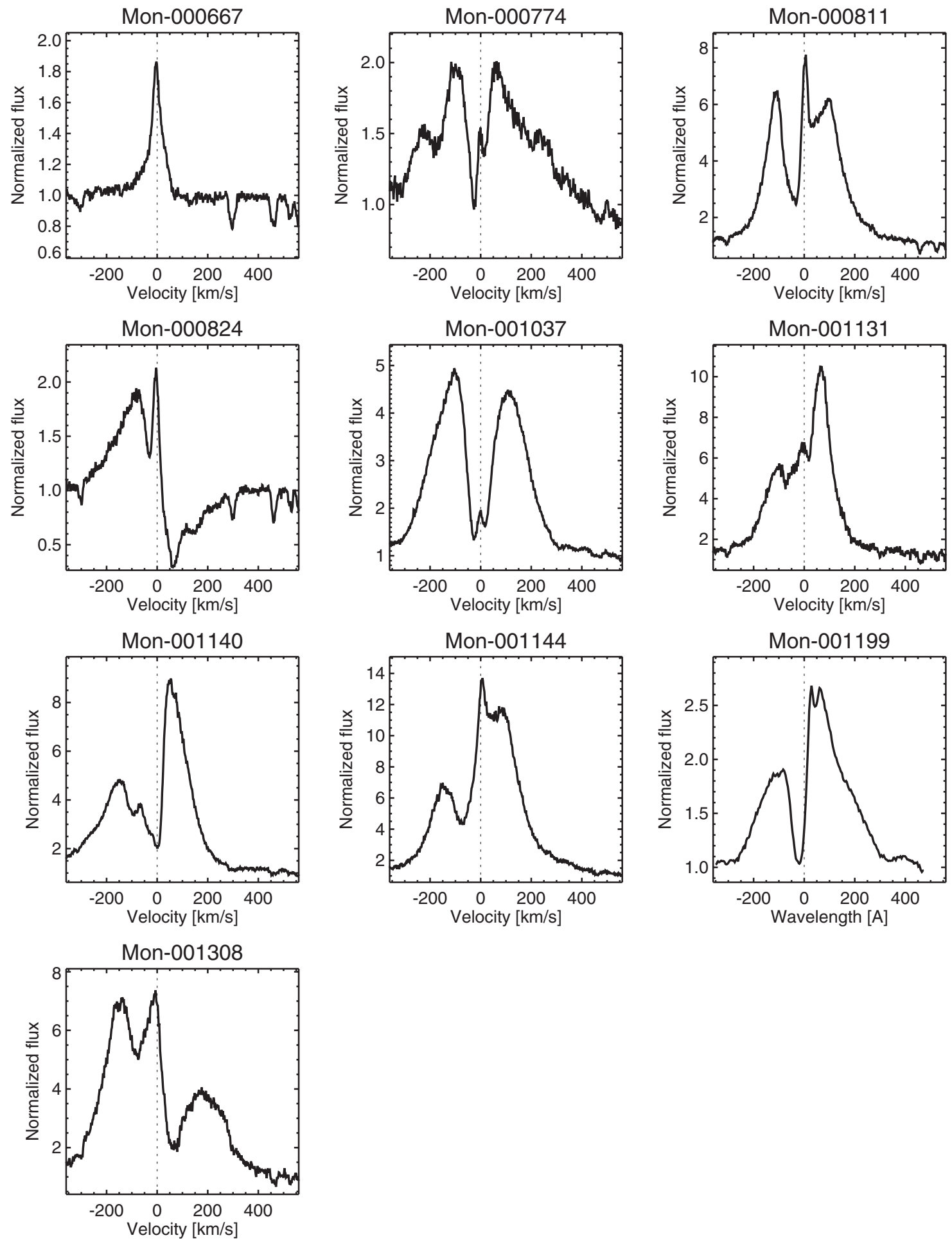

Figure 25. (Continued)

Figure 22 shows the 2011 light curves for which we have both CoRoT and Spitzer data. The center of the $y$-axis scale is set to the median magnitude for each band; the range in $y$ is the same for each of the bands that are shown (for example, if the CoRoT $y$-axis scale was from 14.6 to 14.0, and the [3.6] median magnitude were 10.7, then the Spitzer $y$-axis scale would run from 11.0 to 10.4).

Figure 23 shows the CoRoT light curves for the 12 stars with very large UV excess that were not identified as having accretion-burst-dominated light curves.

\section{A.2. Short- and Long-term Variability of Ha Profiles for the Stars of Table 1}

We have MMT Hectochelle spectra for nearly all of the stars showing accretion burst light curves. For that reason, we primarily show Hectochelle data in Figure 6. The MMT data were obtained six or seven years prior to our light curves; we would prefer to be able to show $\mathrm{H} \alpha$ profiles obtained during our photometric monitoring campaign. For six of the stars of Table 1, we have such spectra from the VLT/FLAMES spectrograph 
(generally, six of the VLT spectra were obtained during the CoRoT monitoring campaign, with the other 14 spectra obtained during 2012 January and February). Five of those six stars also have MMT H $\alpha$ profiles. We can use those five stars to address the extent to which the $\mathrm{H} \alpha$ profiles change on both long- and shortterm timescales, and therefore the utility of using the profile shapes from 2004/2005 to help interpret the 2011 light curves. Figure 24 provides this comparison. The conclusion we draw from this comparison is that, in general, the profile shapes do not seem to change their shapes drastically over either short (days to weeks) or long (years) timescales.

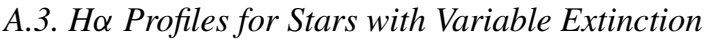 Events in Their CoRoT Light Curves}

In several of the figures in the main body of the text, we have adopted a set of stars to use as a comparison group to the accretion burst group. This comparison set is composed of stars whose CoRoT light curves have prominent flux dips in either 2008 or 2011. In some cases, these are AA Tau analogs, where the flux dips are generally broad and structured and occur approximately periodically. In other cases, the flux dips do not seem to show any obvious periodicity, and we suspect that the variable extinction arises from some type of disk instability. In most cases, the light curves outside the dips shows a relatively constant continuum level. Assuming that these flux dips are due to portions of the circumstellar disk passing through our line of sight, which is the standard model, these stars must be viewed at relatively high inclination.

We have either MMT Hectochelle or VLT spectra of most of the stars that fall into this category. Figure 25 provides the $\mathrm{H} \alpha$ profiles for these stars. Compared to the $\mathrm{H} \alpha$ profiles of the accretion burst group shown in Figure 6, it is apparent that the variable extinction group often has redshifted absorption dips in their profiles, which are completely absent from the accretion burst group.

\section{A.4. Hot Spot Candidates}

A set of stars whose light curves may be dominated by relatively stable hot spots located at high-latitude was identified in Section 7. Table 6 provides basic information for these stars.

\section{REFERENCES}

Affer, L., Micela, G., Favata, F., Flaccomio, E., \& Bouvier, J. 2013, MNRAS, 430, 1433

Alencar, S., \& Basri, G. 2000, AJ, 119, 1881

Alencar, S., Teixeira, P. S., Guimarães, M. M., et al. 2010, A\&A, 519, A88

Allen, L., Calvet, N., D’Alessio, P., et al. 2004, ApJS, 154, 363

Baglin, A., Auvergne, M., Barge, P., et al. 2009, in IAU Symp. 253, Transiting Planets, ed. F. Pont, D. Sasselov, \& M. Holman (Cambridge: Cambridge Univ. Press), 71

Baxter, E., Covey, K. R., Muench, A. A., et al. 2009, AJ, 138, 963

Bertout, C. 1984, RPPh, 47, 111
Bertout, C., Basri, G., \& Bouvier, J. 1988, ApJ, 330, 350

Bouvier, J., Alencar, S. H. P., Boutelier, T., et al. 2007, A\&A, 463, 1017

Bouvier, J., Chelli, A., Allain, S., et al. 1999, A\&A, 349, 619

Cemeljic, M., Shang, H, \& Chiang, T. 2013, ApJ, 768, 5

Cody, A. M., Stauffer, J., Baglin, A., et al. 2014, AJ, 147, 82

Cody, A. M., Tayar, J., Hillenbrand, L. A., Matthews, J. M., \& Kallinger, T. 2013, AJ, 145, 70

Dahm, S., \& Simon, T. 2005, AJ, 129, 829

D’Angelo, C., \& Spruit, H. 2010, MNRAS, 406, 1208

Espaillat, C., Calvet, N., D'Alessio, P., et al. 2007, ApJL, 670, L135

Espaillat, C., Ingleby, L., Hernández, J., et al. 2012, ApJ, 747, 103

Fazio, G., Hora, J. L., Allen, L. E., et al. 2004, ApJS, 154, 10

Fressin, F., Torres, G., Charbonneau, D., et al. 2013, ApJ, 766, 81

Furesz, G., Hartmann, L. W., Szentgyorgyi, A. H., et al. 2006, ApJ, 648, 1090

Grankin, K. N., Melnikov, S. Y., Bouvier, J., Herbst, W., \& Shevchenko, V. S. 2007, A\&A, 461, 183

Hartmann, L., \& Kenyon, S. 1990, ApJ, 349, 190

Herbst, W., Herbst, D. K., Grossman, E. J., \& Weinstein, D. 1994, AJ, 108, 1906 Herbst, W., Rhode, K. L., Hillenbrand, L. A., \& Curran, G. 2000, AJ, 119, 261 Hudson, H. 1991, SoPh, 133, 357

Kulkarni, A., \& Romanova, M. 2008, MNRAS, 386, 673

Kurosawa, R., Harries, T., \& Littlefair, S. 2006, MNRAS, 372, 1879

Kurosawa, R., \& Romanova, M. 2013, MNRAS, 431, 2673

Lamm, M., Bailer-Jones, C. A. L., Mundt, R., Herbst, W., \& Scholz, A. 2004, A\&A, 417, 557

Lima, G., Alencar, S. H. P., Calvet, N., Hartmann, L., \& Muzerolle, J. 2010, A\&A, 522, 104

Long, M., Romanova, M. M., \& Lovelace, R. V. E. 2013, ApJ, 764, 196

Makidon, R. B., Rebull, L. M., Strom, S. E., Adams, M. T., \& Patten, B. M. 2004, AJ, 127, 2228

McKinney, J., Tchekhovskoy, A., \& Blandford, R. D. 2012, MNRAS, 423, 3083

Park, B., Sung, H., Bessell, M. S., \& Kang, Y. H. 2000, AJ, 120, 894

Pasquini, L., Avila, G., Blecha, A., et al. 2002, Msngr, 110, 1

Petrov, P., Gahm, G. F., Stempels, H. C., Walter, F. M., \& Artemenko, S. A. 2011, A\&A, 535, 6

Rebull, L., Makidon, R. B., Strom, S. E., et al. 2002, AJ, 123, 1528

Reipurth, B., Pedrosa, A., \& Lago, M. T. V. T. 1996, A\&AS, 120, 229

Romanova, M., Ustyugova, G. V., Koldoba, A. V., \& Lovelace, R. V. E. 2012, MNRAS, 421, 63

Rucinski, S., Matthews, J. M., Kuschnig, R., et al. 2008, MNRAS, 391, 1913

Samadi, R., Fialho, F., Costa, J., et al. 2006, in Proc. of the CoRoT Mission Pre-Launch Status-Stellar Seismology and Planet Finding, ed. M. Fridlund et al. (ESA SP-1306; Noordwijk: ESA), 317

Siess, L., Dufour, E., \& Forestini, M. 2000, A\&A, 358, 593

Silva Aguirre, V., Basu, S., Brandão, I. M., et al. 2013, ApJ, 769, 141

Silva-Valio, A., \& Lanza, A. 2011, A\&A, 529, 365

Siwak, M., Rucinski, S. M., Matthews, J. M., et al. 2011, MNRAS, 410, 2725

Soderblom, D., King, J. R., Siess, L., Jones, B. F., \& Fischer, D. 1999, AJ, 118,1301

Stassun, K., Mathieu, R. D., Mazeh, T., \& Vrba, F. J. 1999, AJ, 117, 2941

Stout-Batalha, N., Batalha, C. C., \& Basri, G. S. 2000, ApJ, 532, 474

Sung, H., Bessell, M. S., Chun, M.-Y., Karimov, R., \& Ibrahimov, M. 2008, AJ, 135,441

Sung, H., Stauffer, J., \& Bessell, M. 2009, AJ, 138, 1116

Szentgyorgyi, A., Furesz, G., Cheimets, P., et al. 2011, PASP, 123, 1188

Tajfirouze, E., \& Safari, H. 2012, ApJ, 744, 113

Teixeira, P., Lada, C. J., Marengo, M., \& Lada, E. A. 2012, A\&A, 540, 83

Teixeira, P., Lada, C. J., Young, E. T., et al. 2006, ApJL, 636, L45

Terquem, C., \& Papaloizou, J. C. 2000, A\&A, 360, 1031

Walker, G., Matthews, J., Kuschnig, R., et al. 2003, PASP, 115, 1023

Walkowicz, L., Basri, G., \& Valenti, J. A. 2013, ApJS, 205, 17

Werner, M., Roellig, T. L., Low, F. J., et al. 2004, ApJS, 154, 1

Whitney, B., Robitaille, T. P., Bjorkman, J. E., et al. 2013, ApJS, 207, 30

Young, M., Teixeira, P. S., Lada, C. J., et al. 2006, ApJ, 642, 972 